\section{SMART TRAY}

Speculating The Future

New Zealand Dining Experience
JOE LEVY

Victoria University of Wellington School of Design

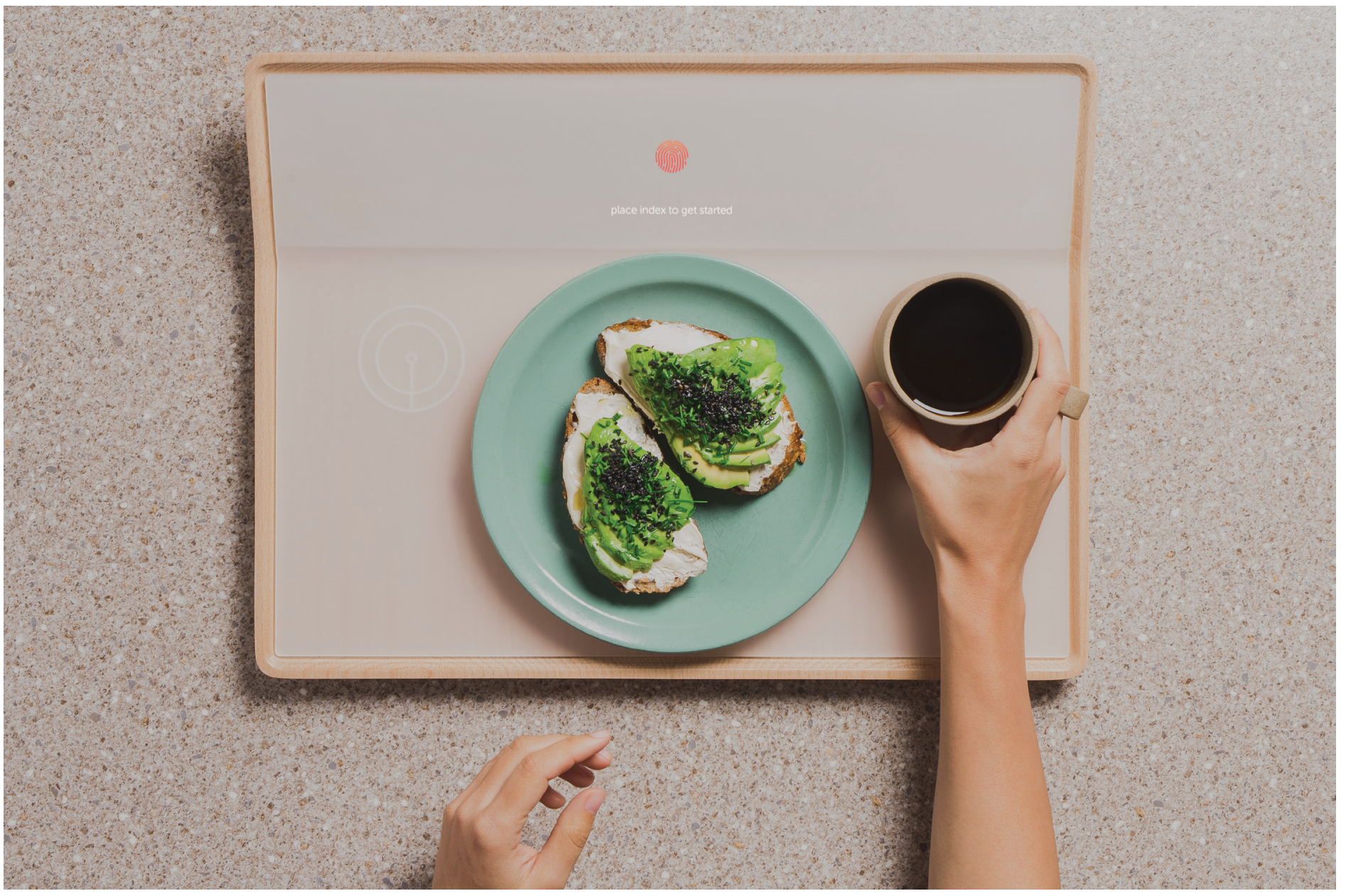


Y 
SMART TRAY

Speculating The Future

New Zealand Dining Experience

University of Wellington

Victoria University of Wellington
School of Design 

Design should not dominate things, should not
dominate people. It should help people. That's its role.

$$
\text { - Dieter Rams }
$$


Upon completing this thesis, and thus six years at Victoria University, I now embrace the chance to say thank you.

To my family, particularly Megan, Ross and Rachel I thank you for the unwavering support you have shown me - its purity is immeasurable.

Tonya Sweet, you embraced the primary supervisor role months into the commencement of this thesis and
for that I am truly grateful. The relentless drive and passion you have invested in this project has been invaluable. Ross Stevens, as secondary supervisor I thank you for being the instigator. The opportunities and guidance you have afforded mever the years are

Nan O'Sullivan - your warm, yet honest support over the last six years has been absolutely priceless. And to the six years has I extend my sincerest gratitute - notably Margaret
Maile Petty, Simon Fraser, Tim Miller, Bettina Neu, Jeongbin OK and Bernard Guy.

A massive thanks must be passed on to the cohort am now fortunate to call friends - James Irvine, Oscar
Pipson. Annabelle Nichols. Andrew Matautia, Matt Pipson, Annabelle Nich
Everitt to name but a few.

It is essential too to recognise the contribution made by the participants of my research case studies.
Although you cannot be named I thank each and every one of you for your time and gusto.

And lastly to you, the reader. Thanks for showing an interest and I hope my work lends an element of
insight or inspiration in whatever capacity you allow it.

Cheers, Joe. 
This research proposes a design solution that embraces New Zealander's proclivity for pervasive and desires of the future Kiwi dining experience. This research proposition is directed by an approach that situates itself between future forecasting and speculative design, whereby the design output is critical reflection about the future of design as it relates to domestic dining appliances. The development of a design solution, the Smart Tray, encapsulates these aims and has been guided by a comprehensive between culture, technology, design and social behaviour.

The Smart Tray seeks to acknowledge New Zealand's history while embodying its contemporary domestic
dining culture in proposing an appliance-device that embraces digital technology as part of the everyday dining experience. This research has been supported by the application of various methodologies inclusive of the clical revew of academic theralure that has functioned to frame and support the scope of
the research proposition; case studies in which a selection of Kiwi households have been interviewed, observed, and their behaviours analysed in order to gain a greater understanding of contemporary dining habits and their relationship with pervasive development inclusive of concept sketching sketch modelling, experience prototyping, and user feedback. Although this research is contextualised within New Zealand, the general research outcomes as a result of this research, including the exegesis and design of the final Smart Tray, are intended to offer a valuable critical perspective and viable future design
solution that will aid in furthering the professional field solution that will ad in
of dining design. 
The New Zealand Kitchen from the Twentieth Century to Today Smart Homes, Pervasive Technology, and the Dining Table

The Social Implications of Tech-Driven Dining

Speculating the Future of Dining Design

PRECEDENTS

METHODOLOGIES

CASE STUDIES

Millennial Generation

Generation $X$

Baby Boomers

Silent Generation

PRELIMINARY DESIGN

Placemat Table Concept

Smart Tray Concept

Smart Tray Iterations

Dining Zone

Smart Tray Prototype

Digital User Interface

DESIGN SOLUTION

CONCLUSION

REFERENCES 
INTRODUCTION

New Zealanders, commonly known as 'Kiwis', are increasingly connected and dependent on ecchnology. The wide consumption of digital tools and applications have found their way into the
Kiwi home where these pervasive technologies have begun to reshape traditional behaviours. In the case of contemporary domestic dining habits, the behaviours associated with dining have seen a shift from a traditional emphasis on socialisation around the dining table to a more individual
experience in which digital interfaces play a central experience in which digital interfaces play a central
role. Socialisation is now often mitigated through social media, and people are increasingly tuned in to screens rather than tuning in to face-to-face conversations during mealtime. Looking ahead to it is reasonable to speculate that our dependence on pervasive technologies will continue to shape our behaviours and taditions and that digit Kiwi households dine.

The inspiration for the development of this research stems from a personal position that was fostered in cy design a better world. This is arguably achievable
denteris through the application of a critical consideration towards human needs and in taking full advantage of our technological developments in an effort to strike a balance between traditional values and future possibilitites. This research was also inspired by an Anvaluable thre-month nhtems hit Fisher\&Payke the professional practice involved in was exposed to of innovative design within a New Zealand context. This combination of educational and professiona experience provided the foundation of knowledge and awareness necessary to successfully direct this research project independently and with a high degree of critical reflection. 
This research proposes a design solution that embraces New Zealander's proclivity for pervasive digital technology and that aims to meet the needs and This research proposition is dituates itself between future forecasting and speculative design, whereby the design output is viable while simultaneously capable of provoking critical reflection about the future of design as it relates to domestic dining appliances. The development of a design solution, the Smart Tray, comprehensive investigation into ged by a

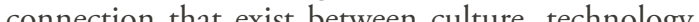
design and social behaviour. The Smart Tray seeks to acknowledge New Zealand's history while embodying its contemporary domestic dining culture in proposing an appliance-device that embraces digital technology as part of the everyday by the application of various been supported inclusive of the critical review of acad nic literature that has functioned to frame and support the scope of the research proposition case studies in which a selection of Kiwi households have been interviewed, observed, and their behaviours analysed in order to gain a greater understanding of contemporary digital technologies at home and itertive desive digital technologies at home, and iterative design sketch modelling experience prototyping and user feedback.

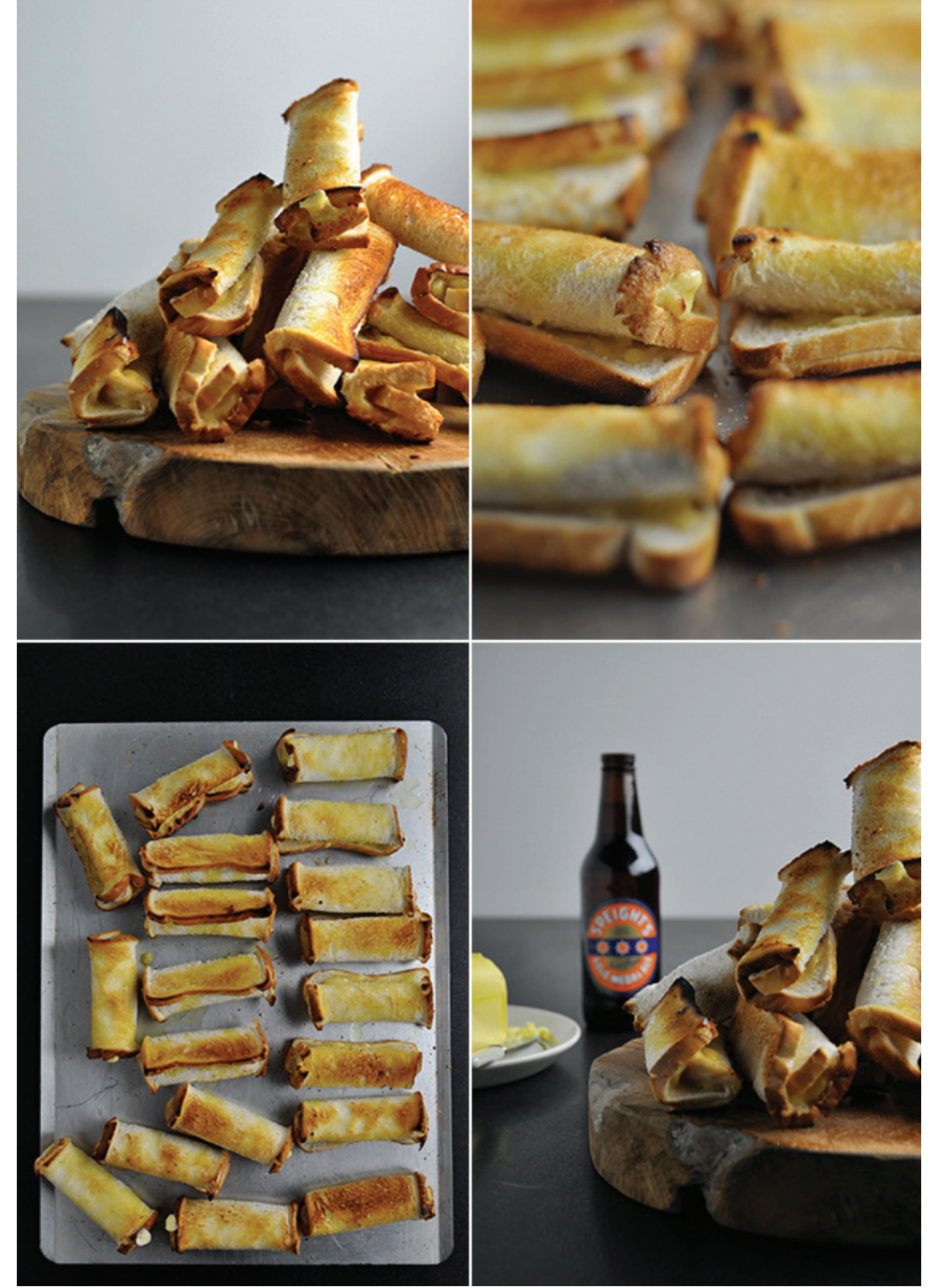




\section{LITERATURE}

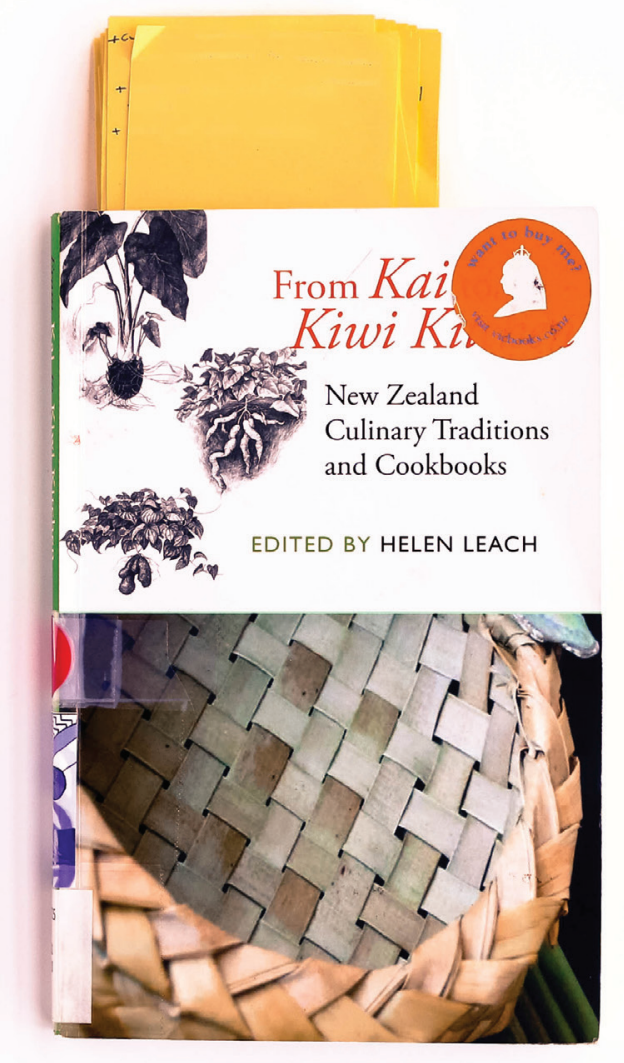

The study of literature has been essential in supporting the conceptual development of this pages has The licion to fow research proporito and methodologes applied as well as to provide a critical understanding of the various topics considered as part of this thesis. This material is presented chronologically - from the past, to the present, and looking onward to the future - in five sections. The first section, From Ka to Kiwi Kichen, prons a histrical overview of terious Kitchen from the Twentieth Century to Today, eviews a brief history of domestic meal prepara according to its designated space within the home and various social implications. Section three, Smart Homes, Pervasive Technology, and the Dining Table, intoduces some of the key considerions New Zealand dining experience The fourth section, The Social Implications of Tech-Driven Dining considers the ways in which technoloyy has impacted

our everyday lives. Lastly, in section five, Speculating speculative desing Design, future forecasting and for exploing and supporing the destopnet of design outcome that aims to meet the future needs and desires of Kiwi households.

In addition to the literature reviewed, this section includes supplementary imagery, and visu precedents inclusive of existing commercial designs within the categorits of interior architecture digital technoles been beneficial in providing a wider understanding and appreciation for the considerations that have given shape to this field of study, as well as inspiring the development of the final design outcome. As a comprehensive whole the visual precedents And literature reviews have supported an in-dept hrough which solutions to complex issues mays realised. 


\section{FROM KAI TO KIWI KITCHEN}

This section provides a historical overview of the origins of New Zealand dining traditions and behaviours.

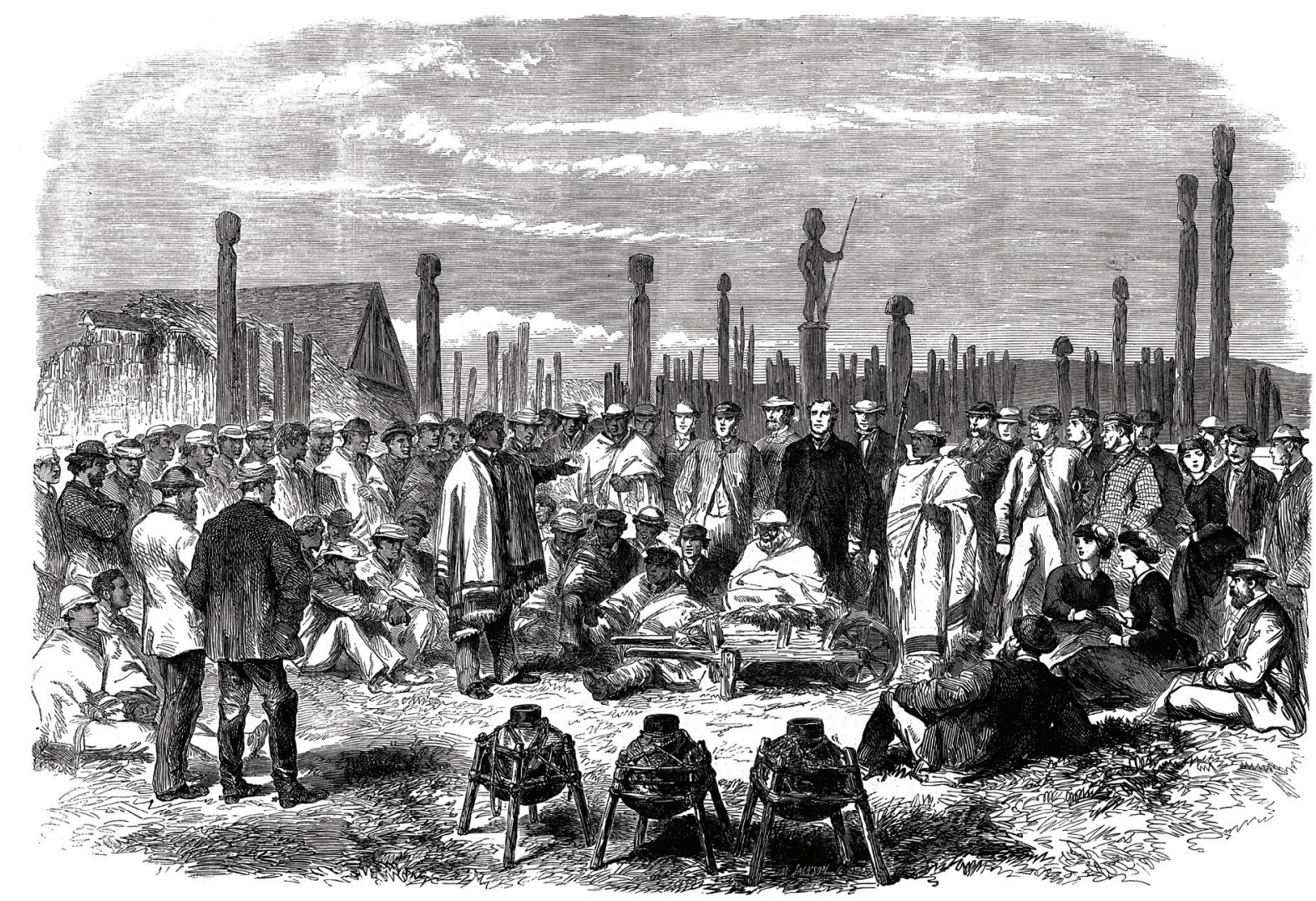

Kia whakatömuri te haere whakamu

My past is my present is my future.

fure with my eyes fixed on my past."

(Mäori Whakatauki)

The Maori proverb above is an appropriate poin of departure for my research: the act of looking back as we move forward is equally important

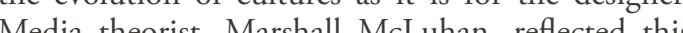
(ame idea when he was quoted as saying "we look at the present through a rear-view mirror. We march backwards into the future" (Hefner 1969). While this research investigates possibilities for the future of the New Zealand dining experience, it deserves thorough consideration of New Zealands short

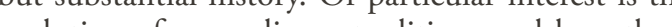

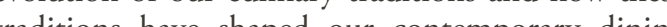
behavious at home, and the subsequent effect this has had on our interactions with household spaces, furniture, appliances, and associated products.

While there is limited academic literature on the tradition, Professor Helen Leach from Otago Un New Zealand has offered sizable her book 'From Kai to Kiwi Kisthe,' Exploring New Zealand's history offers great insight into our cultural heritage, and forming an understanding of the evolution of our national culinary traditions has been an important undertaking throughout tis sesearch. A culhary tradition can be defined as tis colve prosive develow ant apting to chas pood supply new technology, social trends, and external influences (Leach, 2010). A perfect example of this, looking back a few centuries in time, is the first Eastern Polynesian Maori settlers to Aotearo who went through a period of progressive chang

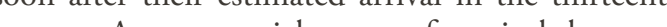

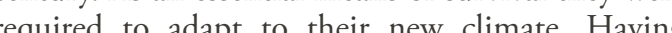
originated from warmer, more humid climates that boasted temperatures between twenty and thirty degrees Celsius, upon landing they immediately met a much cooler climate than their pervious home Leach, 2010). Acting intuitively they replaced weir dwith harvests of 
aptitude for adaptation and progressive change that have shaped New Zealand's culinary traditions: may serve as one of the earliest examples of New The first is the aforementioned beaching of Eastern Zealand resiliency and our culture's renowned "an- Polynesian voyage canoes on our shores in the do, number eight-wire attitude. The same attitide thirteenth century, and the second case being the seemingly saw early Maori not only develop but first recorded arrival of English ships in Norther was observed with interest by British scientists and landing New Zealond wo bound to it predomise crew: "They speculated whether the Maori camping cultural influences: the Maori and the British. A at nearby Purangi River [Whitianga] were eating relations developed between the Maori and Britis roasted fernroot, fish and shellfish because they inhabitants, overtime the culinary traditions bega had been defeated in war and lost their provisions" to reflect a mingling of both cultures. This includes (Leach, 2010). Although the patriotic ignorance the integration of elements centred on hygiene, such of the British in question provides the reader some as the washing of tea towels and tablecloths separately comical reading today, it significantly suggests the from clothes-the timeless binary principle of Tap struggled to maintain an ordered meal structure the traditional British meal structure. It is worth made up of imported food types barely sustainable noting that this meal structure was adopted as a in their new surrounds.

'From Kai to Kiwi Kitchen' identifies two key events more relaxed version inclusive of a three-meal day culminating with dinner that consisted of starters,

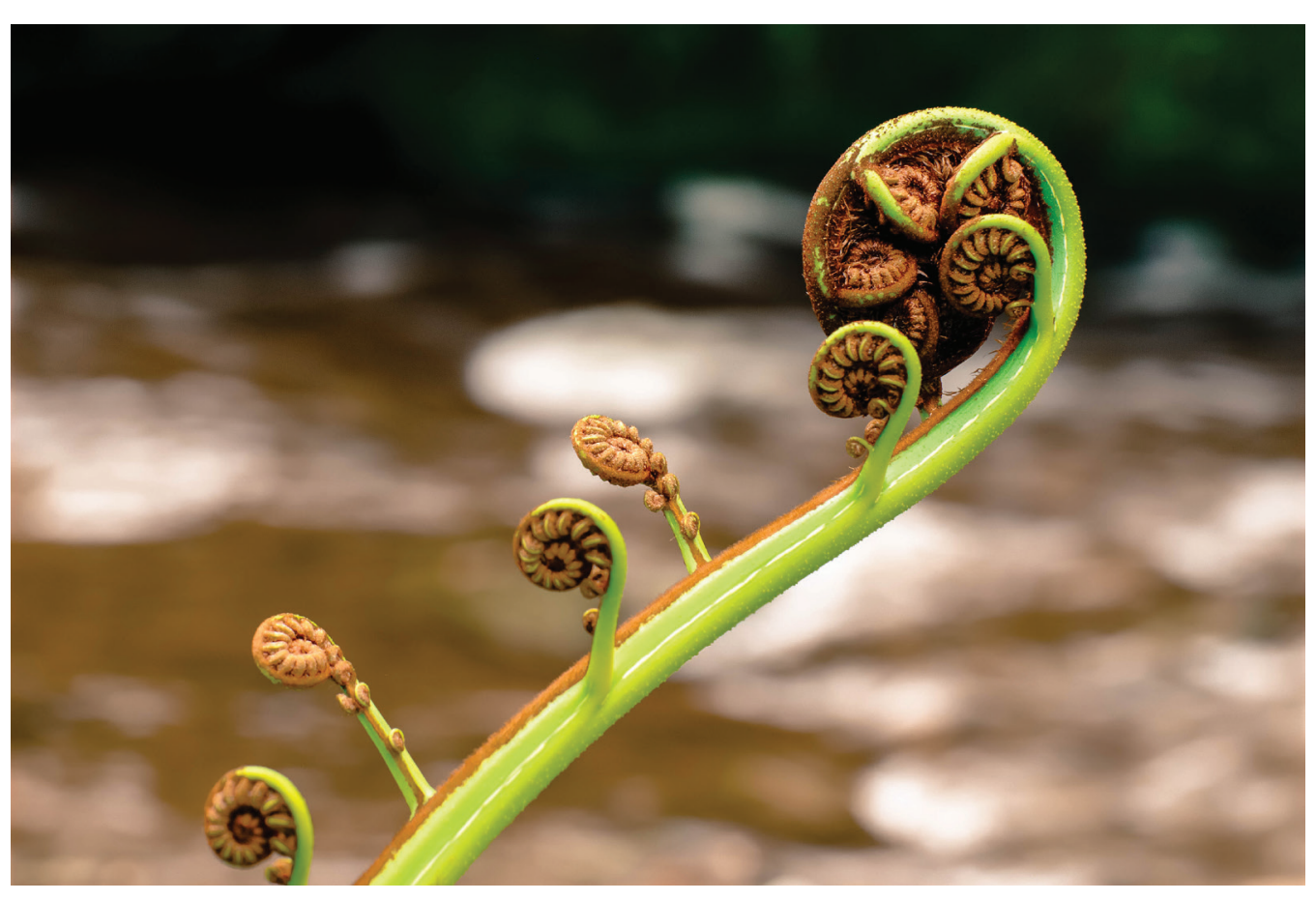

ABOVE: Figure 3. Pikopiko, a Maori term for wild bracken fern, was

\section{THE NEW ZEALAND KITCHEN FROM THE TWENTIETH} CENTURY TO TODAY

This section provides a brief history of domestic meal preparation according to
its designated space within the home and various social implications.
As we enjoy our meals at home it is interesting to acknowledge the link between our culinary tradition does the organistional this hip betws. How kitchen and dining area impact upon one another? What correlations exist between the degree of dining formality with an open or closed plan kitchen? Of particular relevance to this research is the evolving transition of these spaces throughout the twentieth century to today within the New Zealand domestic environment. For more consideration on this contributions of Professer tolen to the academic "Kitchens: The New Zealand Kitchen in the 20 th Century'.

The early twentieth century kitchen's design and layout can loosely be described as a transitional space. Although in 1900 housewives were advertising for domestic households were increasingly taking step tomeards self-sufficiency. As housewives regularly stops on the cooking duties for their household, products and appliances began to reflect the evolving demand for high quality, ergonomic kitchen tools and furniture. This trend has continued in the design of roducts that must meet the decisive demands of a diverse market quat

The evolution of the British home in the twentiet century was also a key influence as select trends were adapted in the development of the modern Kiwi kitchen. As post-war reconstruction commenced in the 1920s and peaked after World War II, the Britis in open-plan interiors. This new intat lesulted addressed the need for better quality and layour of fresh air sunlight and proximity to outdoor aress. It also constructed a sociologically more efficient home, prompting its occupant's greater equality of household roles and the celebration of open-plan, social spaces. Before open-plan living became the arious New Zealand, the Kiwi kichen underwent The Scullery, for example, which reater effer found adjacent to the kitchen and pantry went away along with the servants. The kitchenette made its first public appearance in Auckland real estate publications in 1913 in an attempt to minimise and simplify the kitchen. The end had finally come for large, segregated kitchens with separate scullery
and pantry spaces (Leach, 2014). Instigating 
long-term trend in kitchen design, architects and designers alike continually seek to minimise the size of kitchens with particular focus on improving the efficiency of use for the household it serves. in serving the houschold the seanless integration continued to develop in enhancing the efficiencies of open-plan living.

The evolution of the open-plan kitchen underwent significant developments in the 1960 s with the removal of a wall being key to the layout. In place of the wall was now a pents Kiwi hench and overhead adopted the open-plan kitchen-living area now commonplace in most new construction today It is interesting to consider that up to this point kitchen walls were essential to enclose the space in order to define boundaries around the task and role of mea prepation. A contemporary wall-less kitchen, in contrast, celebrates the seamlessness between no

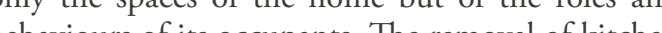
walls has offectively enants. The removal of kitche between the space allocated to meal preparatio with general 'living' and dining spaces. Based on this, one could hypothesise that with the dismissal of traditional spatial boundaries in meal preparation is he dismissal of traditional boundaries related to the enjoyment of those same meals. As the upcoming and 'laid back' attitude, it is little surprise that the formal dining experience (and formal dining table) are rapidly becoming a relic of the past.

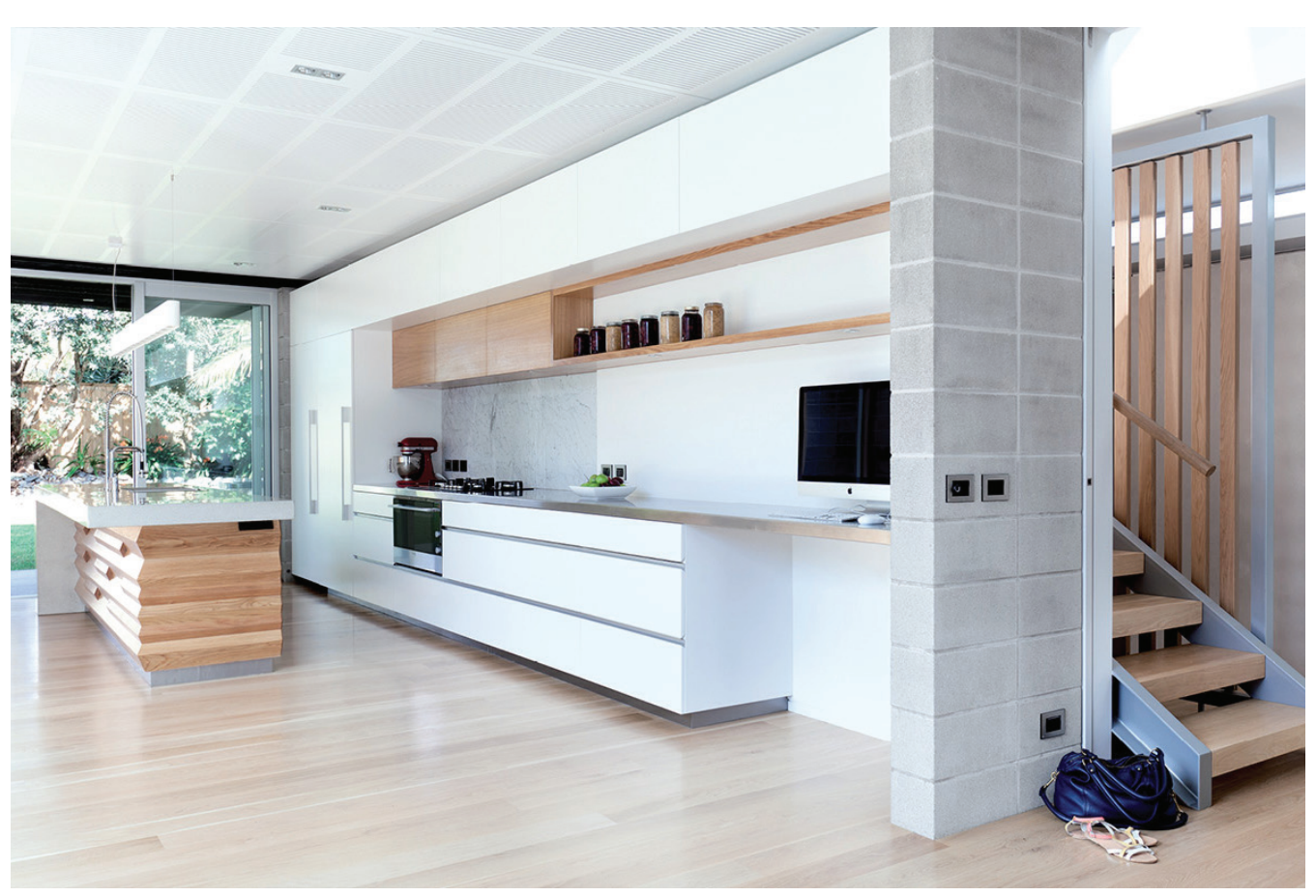
ABOVE: Figure 4 . Contemporary open-plan Kitchen-living space in
Westmere, Auckland by Gerrad Hall Architects, Wood-Tech Kitchens, Westmere, Auckland by Gerrad

\section{SMART HOMES, PERVASIVE TECHNOLOGY,}

\section{AND THE DINING TABLE}

This section introduces some of the key considerations around the application of technology to the future New Zealand dining experience.
No object can compete with the simultaneous functional and symbolic value of a dining table. The

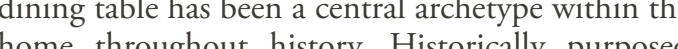
to commune with the Gods the dining table first set out to offer a raised surface in sharing meals, representing the verticalisation of religion and the desire to reach closer to heaven (Kaufmann, 2010). As a result of the changes that occurred between the eighteenth century and recent history, howeve, the role of the dining table has transformed. became a permanent five with a thecific function in the domestic setting. Sharing meals at the dining table became a staple behaviour, and sitting around the table to eat while facing one another became an ethical standard. These are the models that our contemporary notion of the dining table is built today

We live in a rapidly changing period of time. Within the past fify meals at home has drastically fallen. Thanks to longer working days, the evaporation of traditiona household roles, and the development of instan meals and ever-accessible fast-food restaurant twenty minutes a day cooking (Kaufmann, 2010). Our dining experiences and behaviours around

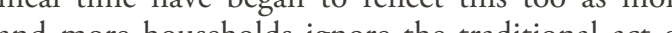
haring a meel at the dining table in preferce of a more adaptable, relaxed dining context. Dining at home today frequently means eating in front of the television or a desktop computer, while potentially also staying connected to the world of pervasive computing through our tablets and moble phones. with

rench Sociologist Jean-Claude Kaufmann offers useful insight into the contemporary dining experience in his research into the power meals have in structuring everyday relationships and the Although written from a French dining at home. ideas expresed provide an applicable framework to better understand contemporary dining trend within a 'Western' context. In his book "The Meaning of Cooking', Kaufman considers the impact of technology on traditional dining practices. Instead of resenting the move away from traditiona 
occurred in contemporary dining. "There is nothing authors Johannes Schöning, Yvone Rogers, and anecdotal about watching television over the family meal. The television plays an important tole and tells us a lot about what is at stake at mealtimes. Meals are part of the architecture of family life, not leas about anything" (Kaufmann, 2010). Since its firs inclusion in the 1950s the TV has led the revolution in integrating technology into mealtime.

Today we not only watch television during meals, but we are increasingly tapped into various pervasive rechnologies. Pervasive technology (also referred to as pervasive computing, or ubiquitous computing microprocessors into everyday objects so they can communicate information. The words pervasive and ubiquitous mean, "existing everywhere", and this is possible through a convergence of wireles technologies, advanced technologies, and the Internet (Rouse, 2010). Pervasive computing devices are completely connected and constantly available tech, and other data-collecting devices that users may or may not be aware of. What is the potential impact of these pervasive technologies to our dining traditions? In their article 'Digitally enhanced food Antonio Kriger speculate on the design evolution might combine computational and digital aspects of food design, technology, and engineering. With the advent of pervasive computing environments the opportunities are emerging" (Schöning, Rogers \& rüger, 2012).

Many of these pervasive technologies are already in

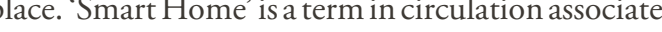
dom the equ refers to home automation energy costs interty wireless devices, entertainment centres, and security (Bell \& Kaye, 2002). In the case of dining, home automation, interactive appliances, remote controlling, wireless devices and entertainment in es inclusive of pervasive technologies are found growing trend, it is not diffcult to predis to this use of advanced pervasive technologies will persist in the future home environment and in particular our approach to the dining table. Author MG Siegler, blog writer for TechCrunch and general partner a

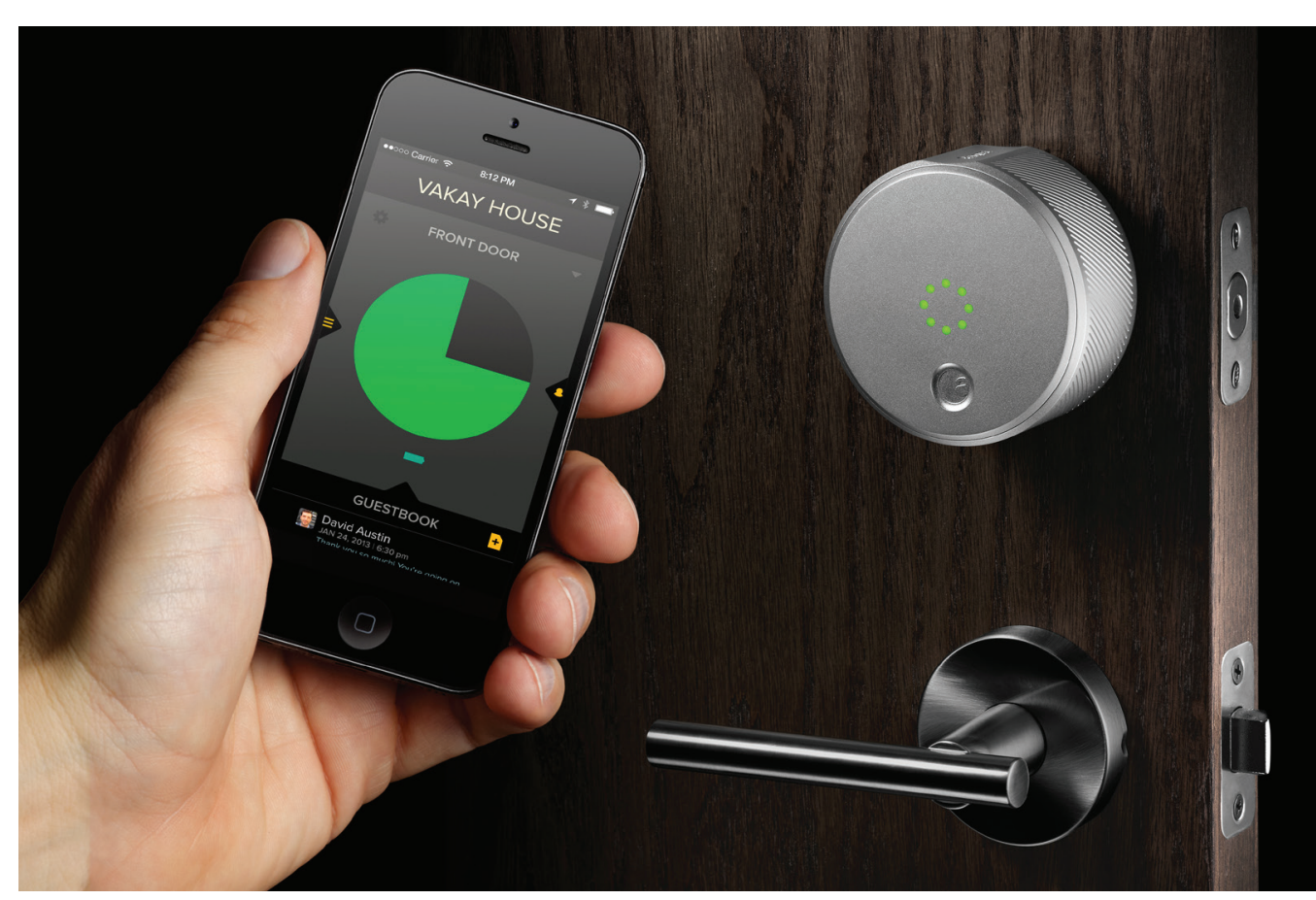

ABOVE: Figure 5. Smart Lock by home automation startup Augus Is an example of pervasive technology's integration within
Google Ventures, foresees the merging of tables with technology

"What's more likely? In ten years, everyon goes to a restaurant and talks to one another without pulling out their phones at the table way to enable you to more easily use your phones? That's an easy one." "Forgive me, but it's Dinner 2.0. And again, I'm having more fun at these dinners than I ever have. Is part of it antisocial? Sure. Can it lead to distractions if you read a work-related email this is the way the world wols now. We lways connected and always on call. An some of us prefer it that way" (Siegler, 2011).

As pervasive technology continues to integrate itse within our daily lives at homeit is essential to critically assess its sociological impact and effectiveness. Of with pervasive technology and the unilise we shafe capabilities to enhance dily functions withor becoming socially destructive. As designers work to negotiate the increasing reliance and expectation around the accessibility and fluid integration of pervasive technologies, they must provide solution that critically consider the implications of the design decisions on ther human users. Commo f experience design (XD) to the design process is valuable in realising empathetic, user-centred design solutions. Tom Wood of Norwich-based XD design agency Foolproof defines experience design as a design practice focused on human outcomes, particularly the level of engagement and satisfactio that the user dof the for a preduct or senvec and

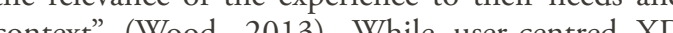
design commonly focuses on sociology, it is worth noting that there are also implications of this design approach on culture. Mike Press and Rache
Cooper in their book 'The Design Experience offer asseciatio folloen culture and experience design in the confronted by a low stool, pastic bom and small hand towel. What dool, plastic bowl and What does all this mean? This is bathroom in Japan, and you are desperately trying o understand Japanese culture" (Press \& Cooper 2003). By introducing specific artefacts into a space we have the power to indicate and provoke a new understanding of culture, interaction, and behaviour

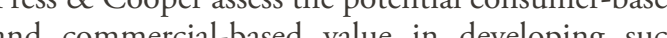
experience driven designed solutions: "Experientichbased design seeks to so thoroughly understand the that he or she perceives as defining. It then fashion product and marketing design strategy to mirro hat experience in a brand culture underlying the product and inspiring passion. A successful brand perte to connect on an intuitive level with the consume's experience of the dining object" (Press \& Cooper,

Considerations situated within an $\mathrm{XD}$ approach have been applied to the design development stage of this research, particularly in proposing a critical and esires of Kiwi households essentilly sechers deliver complex technology with utmost simplicity. This solution intends to celebrate the significance of our Kiwi culinary traditions while envisioning a future understanding of the New Zealand dining experience reflecting the foreseeable needs of individual users wing particular emplasis on their the integran of dining guf applications, and identified as a dining table, with pervasive technology. 

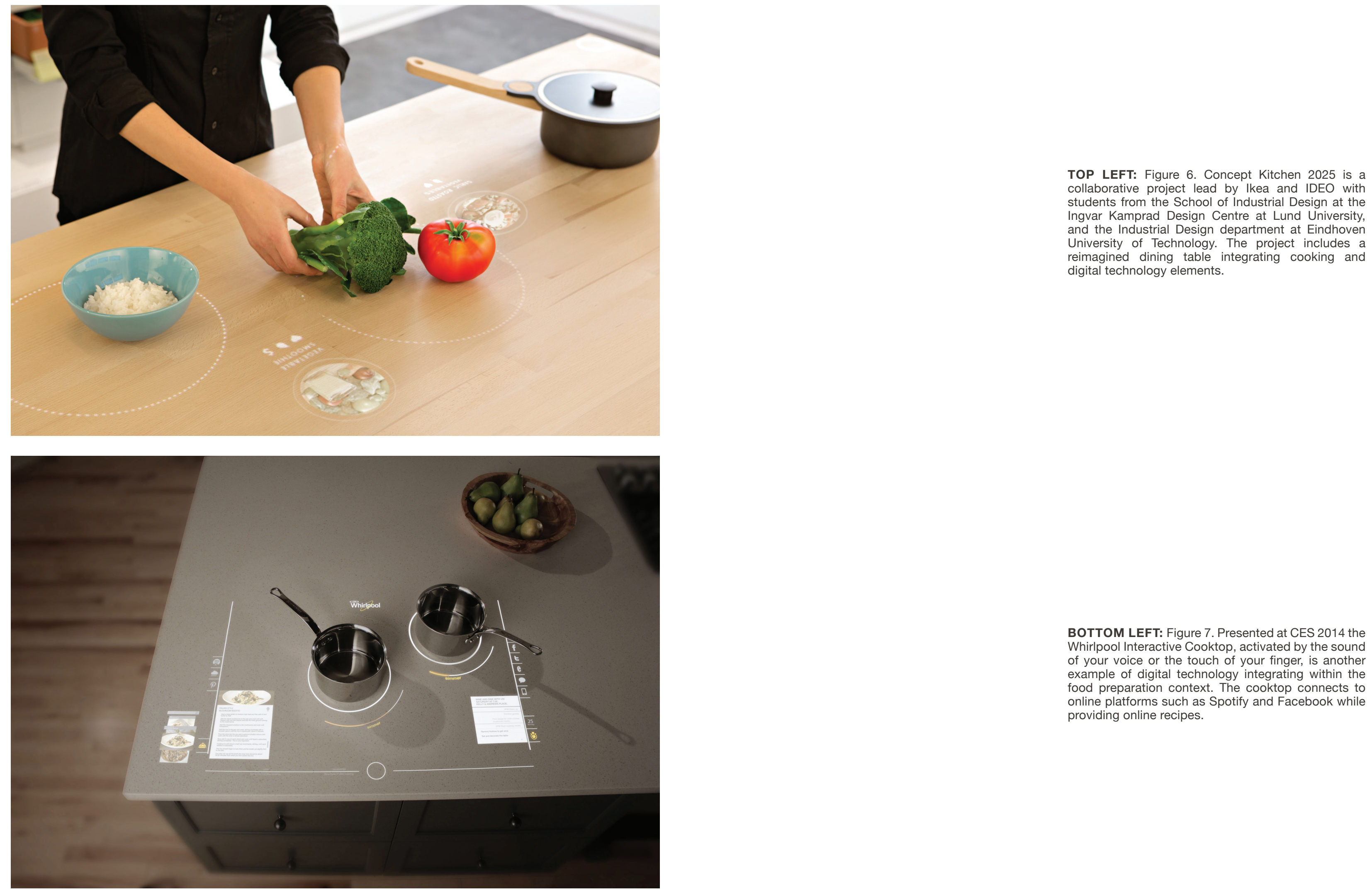

BOTTOM LEFT: Figure 7. Presented at CES 2014 the of your voice or the touch of your finger is snothe

example of digital technology integrating within the food preparation context. The cooktop connects to providing online recipes. 


\section{THE SOCIAL IMPLICATIONS OF TECH-DRIVEN DINING}

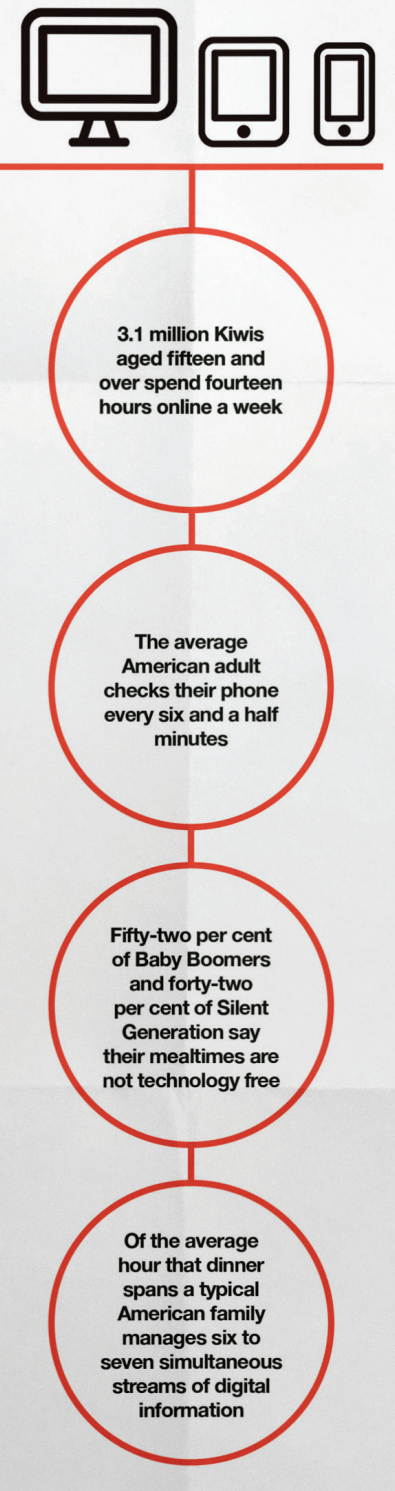

This section considers the ways in which technology has impacted our everyday

Conversation is a fundamental human value, a tool we use to form relationships, learn, discuss for intimacy comes our experiences and desire emaining a key element in constructing our sense of empathy (Turkle, 2015). While technology affords society a wide range of alternative formats for communication and connectivity, the impacts of digital technology on social relationships have often sought negative connotations as an unhealthy distraction. According to Sherry Turkle, Professor the Massachusetts Institute of Technoloy in her recent book, 'Reclaiming Conversation: The Power of Talk in a Digital Age', we should not become antitechnology but rather pro-conversation. As Turkle explains, we still want to be with each other but today we also want to be elsewhere, digitally connected to wherever we choose as we now have he opportunity wherever we (Tulte 2015). We have lenred to place significant value on his co bectivine more so obvious than our sidearm smartphone, now an indispensible utility to our everyday lives. Increasingly this level of technological dependence is also inundating our homes.

Connectivity, however, comes at a cost. Turkle claborates of this point by identifying an iconic Chelsea, of he is on sum "when firten-yedrold free camp describes her disappointment wheher father interrupted their dinner during parents weekend by looking things up on his phone (Turkle, 2015). Admittedly doing the same to her friends outside the device-free camp, Chelsea hin that removing digital tech made her realise the intimate importance, and empathetic yearning she socil bour of technology and now, in the year 2016, to have a conversation with family and friends, colleagues and lovers, we simply pick up our phone. It is worth noting that a conversation may actually consist of text messages rather than a verbal dialogue, and tha we may engage in multitasking while "conversing" on technology on our aility to pay attention to once nother:

"We now rarely give each other our full attention, but every once in awhile, we do. We forget how unusual this has become, that many young people are growing up withou 
ever having experienced unbroken conversations either at the dinner table or when they take a walk with parents or along" (Turkle, 2015).

To provide a clearer scale on contemporary connectivity the average American adult checks their phone every six and a half minutes. Every morning a quarter of American teenagers connect to a digital tech device within five minutes of waking, while eighty per cent sleep with their phones. And if we look more closely at the dinner able and the in far hily simultaneous streams of digital information acros phones, tablets, laptops, a desktop computer, and a TV (Turkle, 2015). Contemporary household are now not only connected, but consuming and interacting with multiple devices simultaneously. To contextualise this specifically within New Zealand 3.1 million Kiwis aged fifteen and over spend working days. And of that 3.1 million, two third access digital content on a smartphone and over quarter via their tablet (Boyte, 2016). According to the Nielsen Company's recent 'New Zealander Connect Consumers Report', our desire to be connected online is and will continue to increase. Americans only would be incorrect.

It is not only teenagers and young adults who are ddicted to technology: Although there is a common misconception that the younger generations are more distracted by their devices at mealtime than Baby Boomers and forty-two per cent of Silent (their mealtimes are not echnology free, compared with forty-per cent of Technology at Mealtimes, 2015). This particul point has guided the identification of case study groups within this research.

Looking to Experimental Psychologists Charles Spence and Betina Piqueras-riszman and their 2013 advancement of future pervasive technology within the home, and dining in particular, is addressed \& Piqueras-Fiszman believe that a tremendous opportunity exists to revolutionise our eating and drinking experiences and behaviours throug innovative integration of food and drink with the Fiszman, 2013). The authors highlight "the of bringing digtal technology to the dining [that] relates to the possible use of the dining table as intelligent 21st century plateware" (Spence \& Piqueras-Fiszman, 2013). This notion of allowing the tablet to inspire future plateware and to enable an interactive "table" has been a key departure point 列

In linking the ideas around the development of a 'smart home' with a sociological awareness of the impacts of pervasive technology this research has also benefitted from a review of the article 'Evolution towards smart home environments, by Tilu Koskel the Kass Vanome-Vainio-Mattila. In this article he authors evaluate alternative digital user interfaces eniron by invesige and requirements of a smart home through the application of an ethnographic study (Koskela \& Vaananen-Vainio-Mattila, 2004). The structure of this study has been adopted in the case studies conducted as part of the research disseminated in this thesis. In the article the authors consider the control of household devices and moreach to the in the context of this research, a device tha connects dining with greater integrated digital connectivity and general control of pervasive technologies within the home. (Koskela \& Vaananen-Vainio-Mattila, 2004). These ideas have been very infuential in the development of the design outcome pursued withi

To summarise, it is imperative to acknowledge the Tignificance of social structures that support family ime, intimacy and empathy with family or friends, while it is equally important to contend with reality of the increased presence and reliance of digita rechnology within the home and our everyday lives. With the application of a critical, user-based desig design solutions that provide increaped to creace hrough pervasive technology while upholding traditions and values that are central to our social well being.

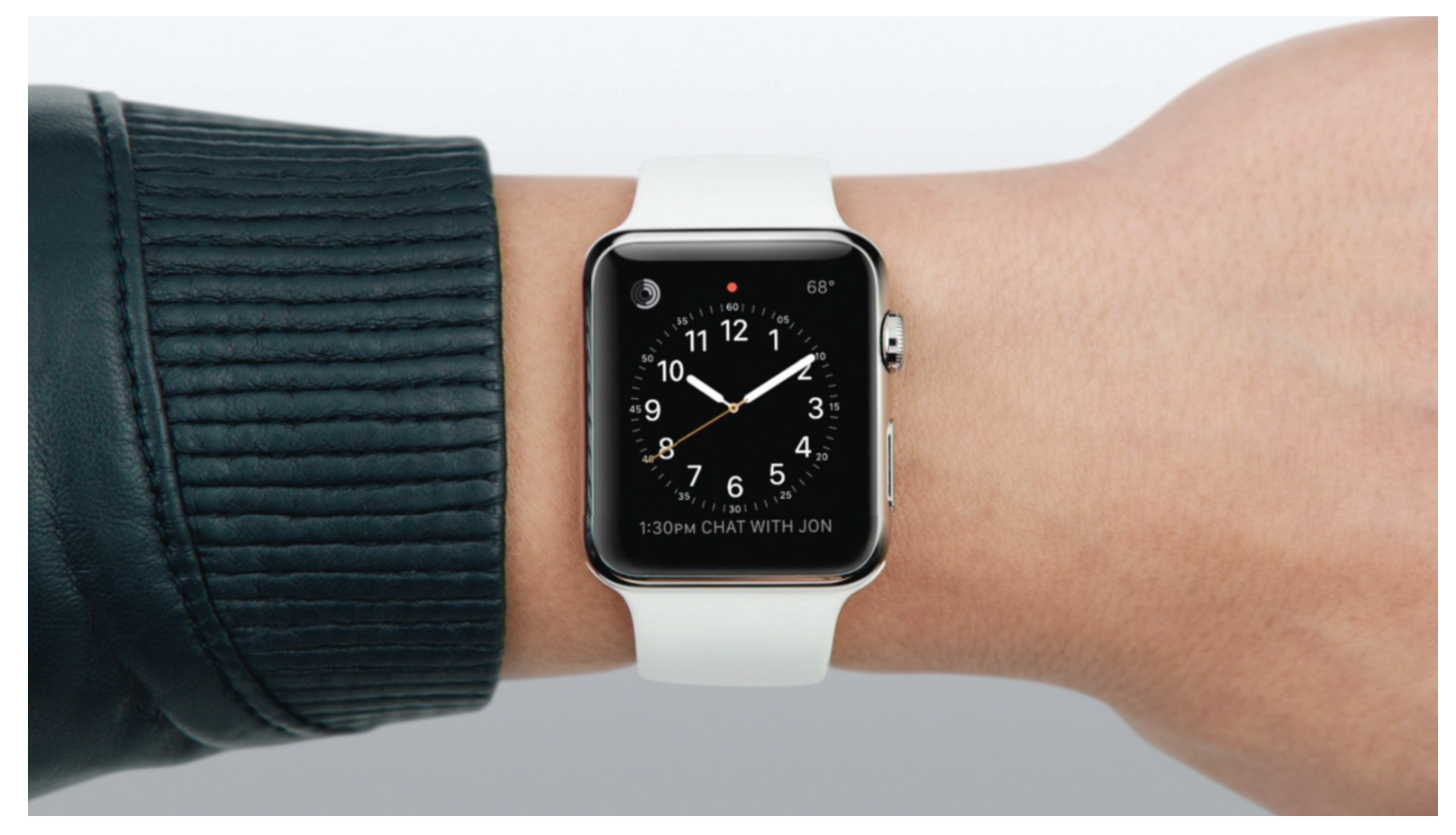


SPECULATING THE FUTURE OF DINING DESIGN

This section introduces future forecasting and speculative design as avenues for exploning and supporting the development of a design outcome that aims to

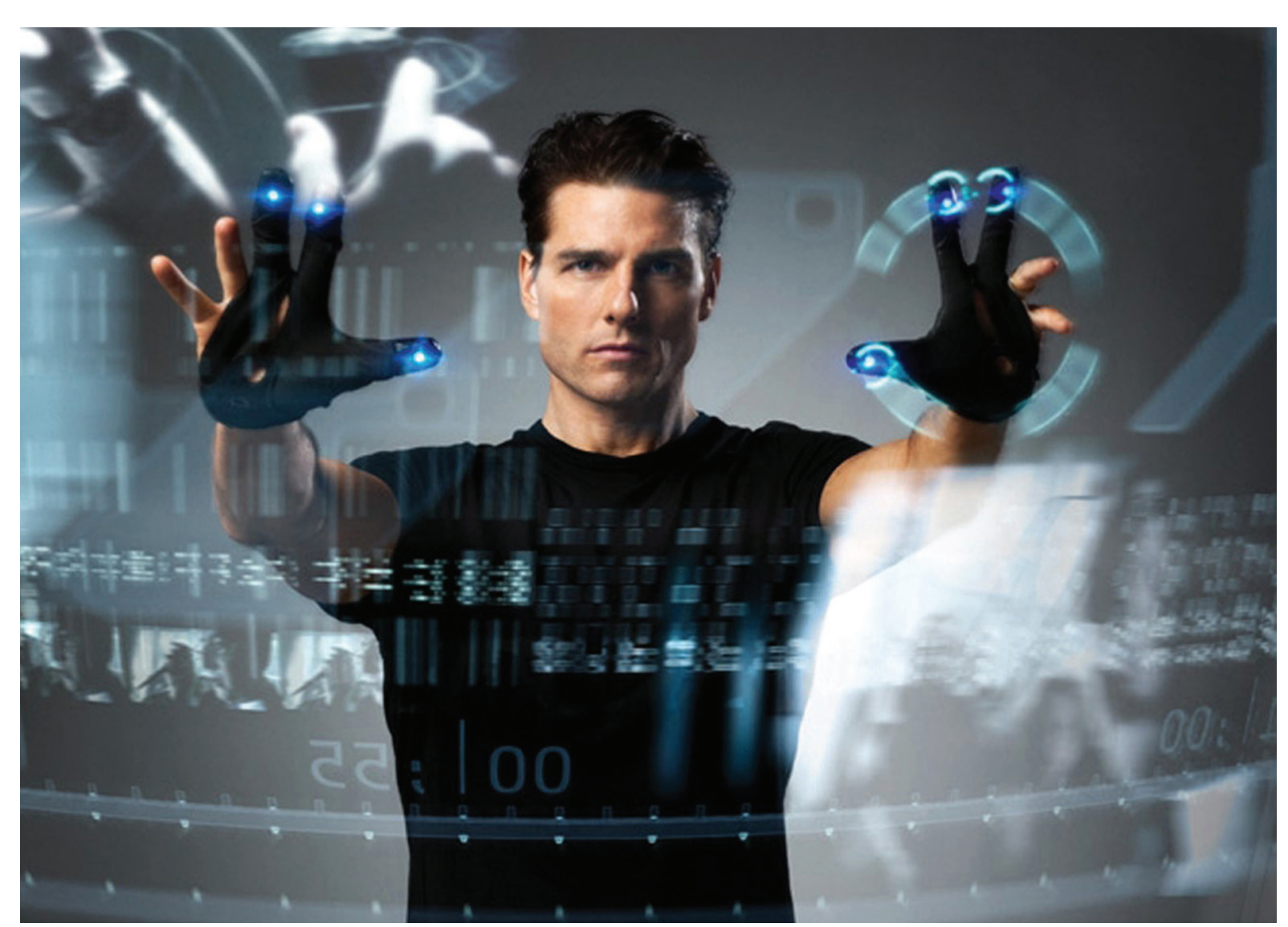

As a sector subsumed in rapidly changing and innovative development, it is safe to assume comparal technogy wit is sine to a constant In the field of design we are required to look beyond the products and services available to us today in magining how to meet the needs and desires of the future. In professional practice the imagining of design solutions for the years ahead is commonly referred to as future forecasting, of which CEO of International tie Kjarecasting consultancy Kja

"A crucial way to be informed about society and understand people, their behaviou needs and mindset, and how that could mpact the future. There are Macro trends (five to ten years) and Micro trends (one to three years). These trends ideally should (Sheppard 2010).

In addition to applying some of the strategies identified in future forecasting, the development of this research has benefitted from considering the value of speculative design as an approach to future. Speculative design thrives on free flowing imagination and he development of fresh perspectives in creating of being (Dunne \& Raby 2013). Unlike furs ative design practice does not aim to solve problems through the development of commercialisable outcomes; instead it strives to interrogate future possibilities and provoke critical discourse about what might be. "Its main purpose is to make us think. But also to raise awareness, expose aster in or film" (Dunne \& Raby 2008), Restre authors Anthony Dunne and Fiona Raby admit that their work usually relies on future forecasting as way to identify new trends or to simply challenge narrow assumptions on the role products play in everyday life (Dunne \& Raby, 2013), yet the futures predicted through this approach, although probable, are usually based on scenarios regarding the rapid changes caused by technological advancements the research explored within this thesis has identified parallel trajectories from which to draw from the two approaches of both future forecasting and speculative design. 
The media entertainment industry has a long tradition of utilising speculative thinking and fictional forecasting in the framing of imaginary futures that have the potential to manifest rea example that effetively showcases the application of speculative innovative technology is found in 2002 episode of the science fiction television series 'Star Trek'. Authors Schöning, Rogers, and Krüger reflec on this episode in their article "Digitally enhanced food":

“... Captain Jonathan Archer and his

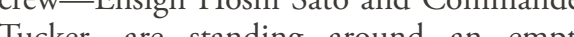
table. Commander Tucker asks about the table, and Sato responds that it's a "molecula synthesizer of some kind —-similar to a protein sequencer." She then asks the table for some cold water; they hear a swuz sound and a glass of water appears. Commander lucker plate of fish appears. This Stre Trek seene, taken from a transcript of the TV series is futuristic. However, this type of application is becoming more feasible with recent advances in technology" (Schöning, Rogers $\&$ Krüger, 2012.

Notable contributions from the film industry nclude the gesture recognition scenes from the use of augmented reality in 'Back to the Future' (Zemeckis, 1985). These are prime examples of how critical fiction inspired advances in technology tha were eventually developed into commercially viable systems. This suggests that the future is imminent syd that it only neds to be inagined to becone below:

The lead production designer for Steven Spielberg's Minority Report, Alex McDowell, in reflecting on his work as well as its subsequent standing as a leading speculative fiction film envisioned an entirely functional city that included transport infrastructure, social, political, and culturat systems, is aware of the impact of his creations in "the ability) to influence the way things develop" (Fairs, 2015). In identifying one piect the most influential to modern society McDowell indicates "the gesture system in Minority Report is the best example lve come across yet" (Fairs

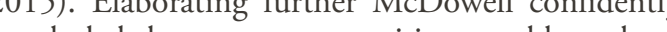
evolved the way it has with as the 'spatial operating environment' supplied new pathways for technoloog innovators, graphic designers, and interaction designers alike to dream

Back to the Future Part II was released in 198 and is now infamous for its depiction of 2015 Amereal De fin did net Zemeckis has recently the future speculative elements from the film such as ubiquitous cameras unmanned drones flat pane televisions and hover boards can now all be found within the contemporary marketplace. Founder of design studio Fuseproject, Yves Behar states, "thes types of films do not guide design, but rather, the (Winston, 2015). Behar concludes new ways of inventing the designe's role is we find utopian aspects of innovative, speculative thinking come to life. Speculative design, and thus future forecasting, seems to want to provoke reason by providing a mixture of fantasy and opportunity.

As a result of digital technologies, industrial designer are now able to create working, material prototypes more easily able to address social and political issues and communicate their ideas through the medium of bespoke designed objects. Thes must reflect necessity and provoke desire of use where the critical study of market segments and their engagentent in user testing processes is an essentia abje his in 'Fictional Functions and Function Fiction' (2008) stating the presenting of impactful designed objects is:

...About a designer being involved in the definition of values that are embedded in an object. Questioning the implications of ideas product. With clectronics we are no simp talking about form and visul aesthetics, by the function of the product, and what it allows us to do and what it prevents us from doing" (Dunne \& Raby, 2008).

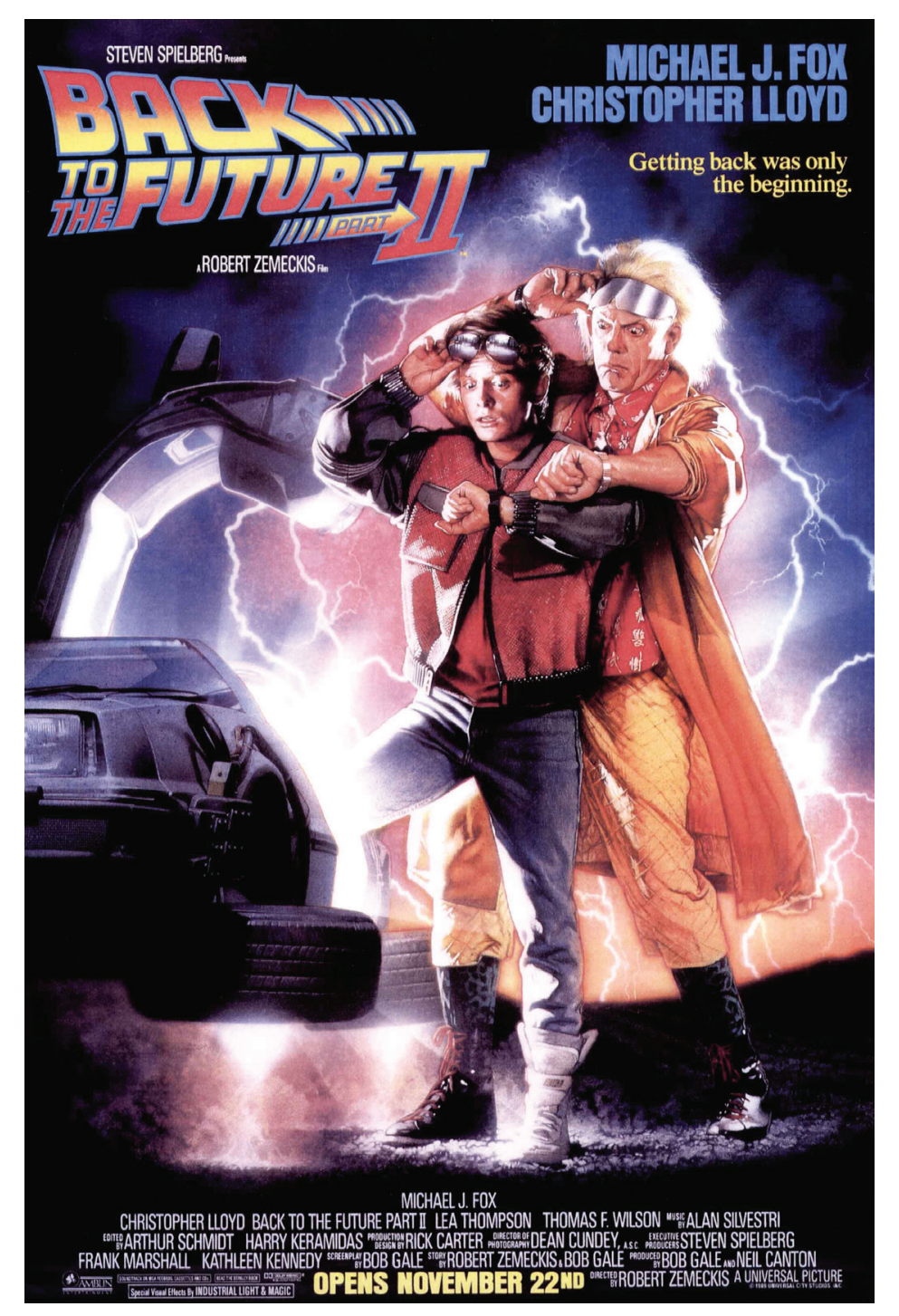



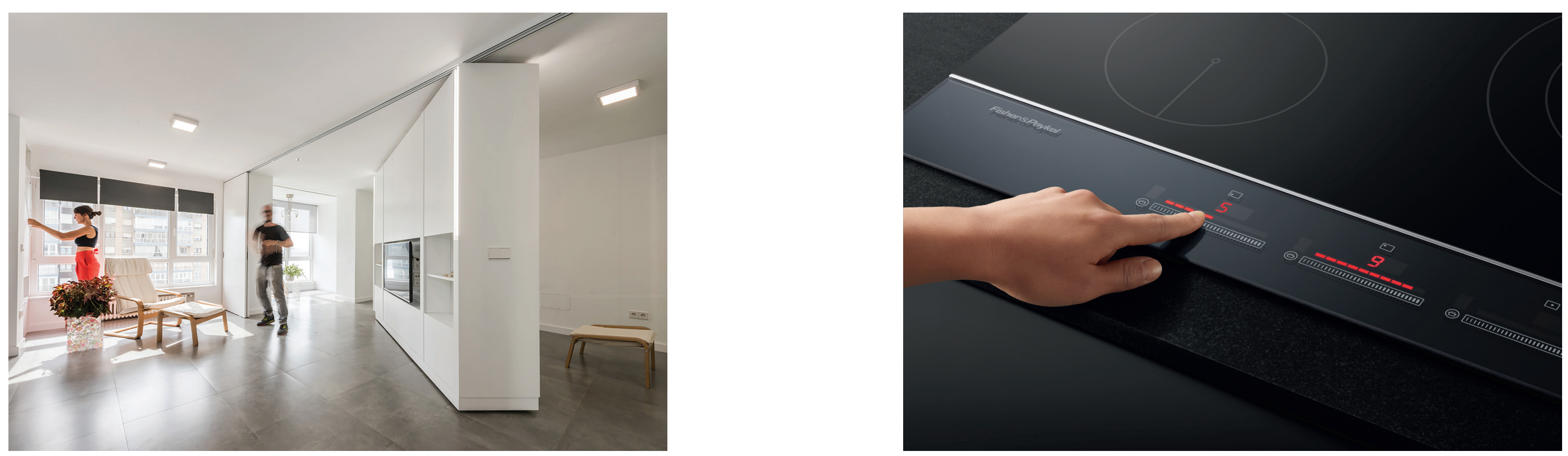

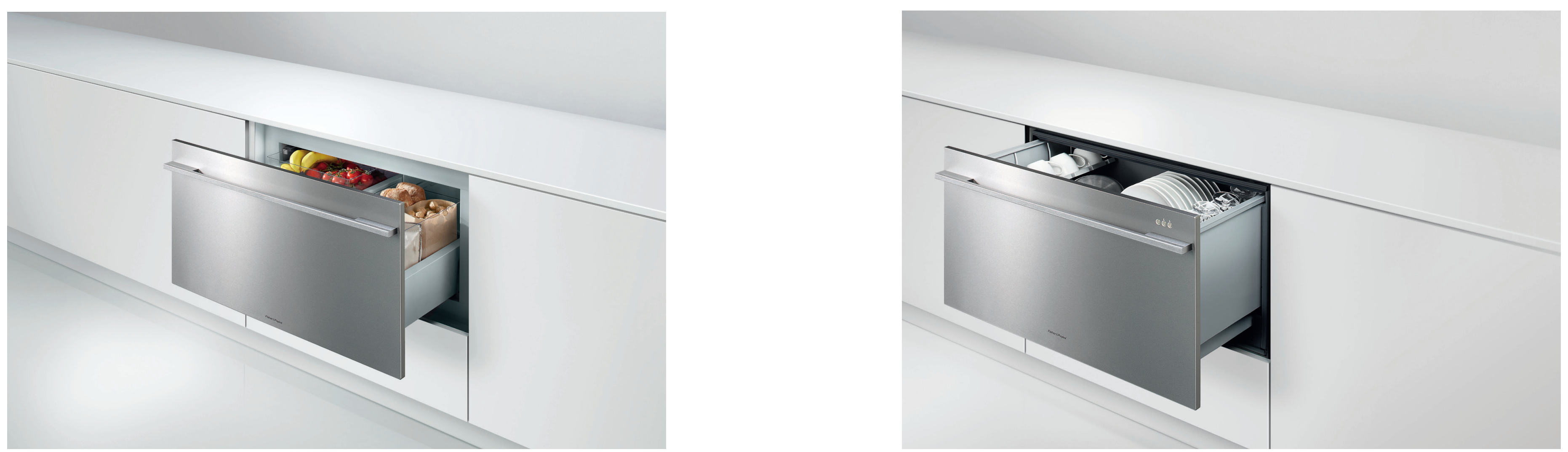

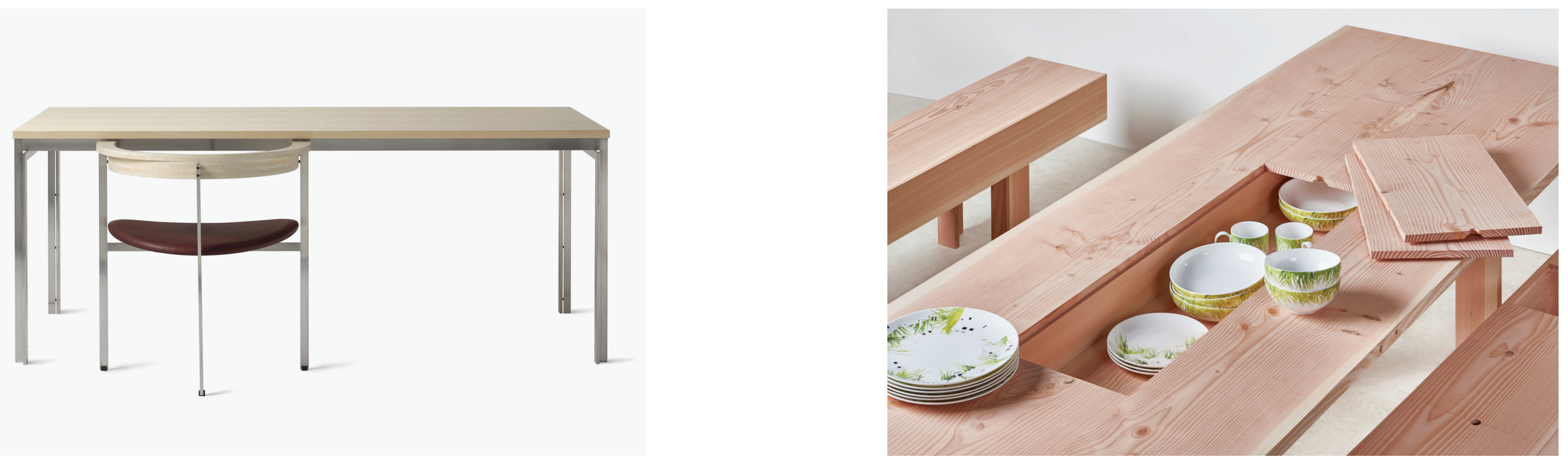

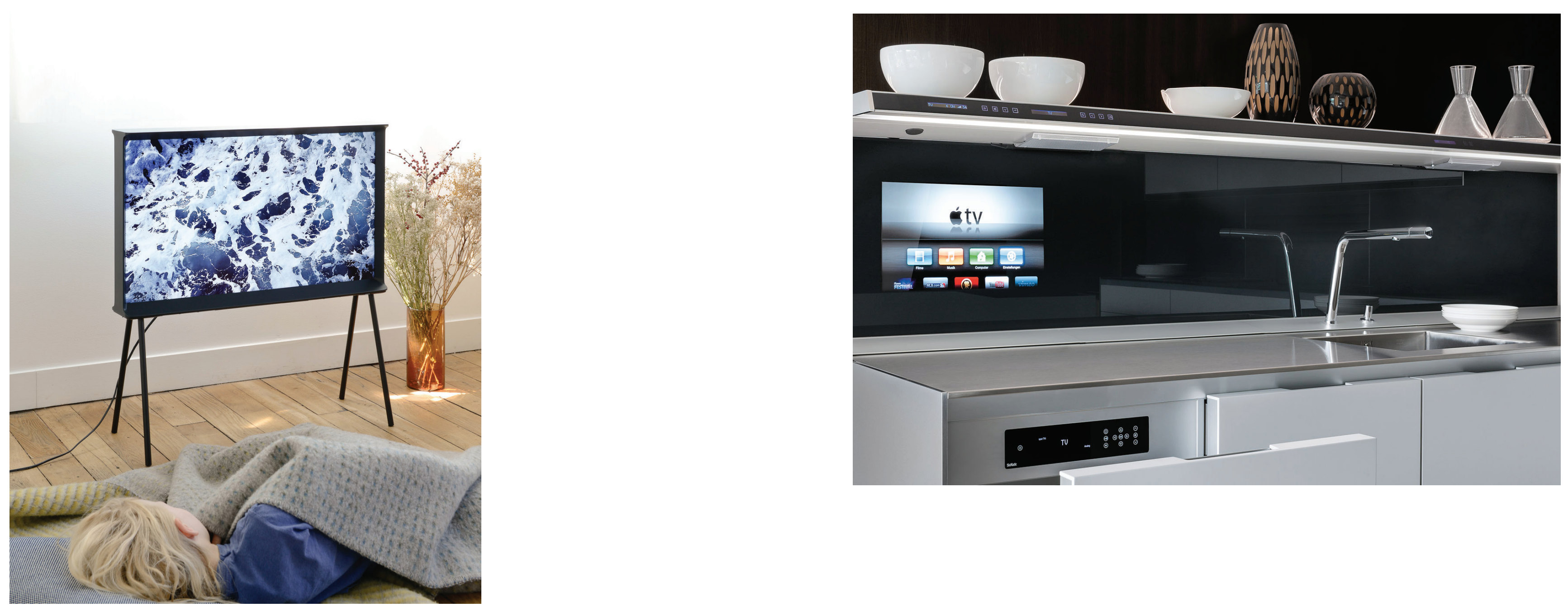

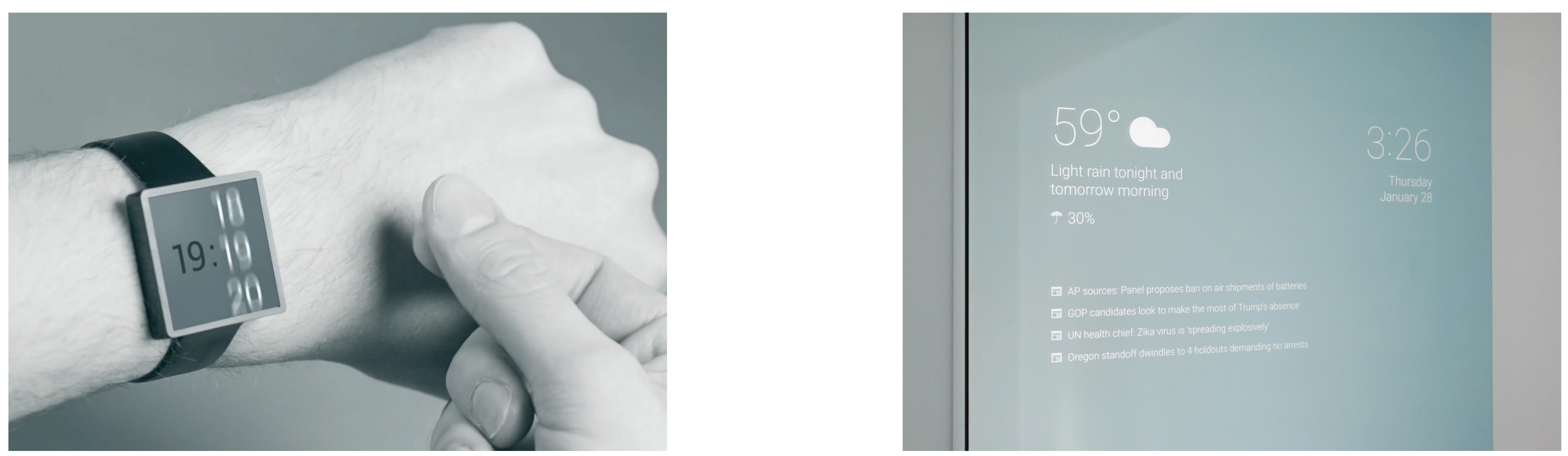
METHODOLOGIES

The methods applied to this research were chosen based on their capacity to support the intentions of the research in considering the impacts of technology on the New Zealand domestic dining
experience and the proposition of a design solution experience and the proposition of a design solution
that aims to meet the future needs and desires of Kiwi households. The methods applied have been categorised according to the three predominant modes of research: literature reviews, case studies, and design development. 
Literature reviews have aided the research in regards to contextualising New Zealand's culinary history and evolution of its kitchen and living spaces throughout the twentieth century. This is essential in investigating and observing contemporary contexts as a means to consider appropriate elements past and present in suggesting designed solutions for the future. In doing so it has been concluded that visiting households in order to observe and participate in
everyday Kiwi dining experiences would be the most authentic approach, presenting the research as a case study series.

The aim from the outset was to visit the widest variety of households possible identifying four groups of household types based on age and X, Baby Boomers, and Silent Gen, Ginetritese household they offer the most variance within elements such as dining behaviours, appliance use, and digital technology etiquette while still reflecting an average Kiwi household without emphasis on elements that include race, religion, or income. Four methods were used within each case study at various stages of the interviews, movement mapping and personac.

These methods have been influenced by and loosely adopted from Damjan Obal and Emilija Stojmenovas research paper "Experience to
Understand" which investigates dining design Understand' which investigates dining design
evolution in providing an effective anthropologically evolution in providing an effective anthropologically
based precedent for research into household kitchen interactions and data collection methods. Forming a twenty-nine-month study vising the resulting methodology was termed EPUI: exploration, participation, understanding and integration - although when broken down these terms result in the methods identified above. Obal a method ava validate the wealth or adopting such designing for the end user and developing user experience research as an essential element to a user experience design process. Parallels can also be drawn between user experience design processes and speculative design practices, as the aim to speculate future design solutions must evoke strong user
Participant observation is extensively used across technique, offering easily readable content that nthropological and sociological studies as an excludes emotions, half-sentences, or garbled speech. effective qualitative method for collecting data on people, processes and cultures (Kawulich, 2005). For this reason the research method has been utilised to gain a better understanding of contemporary New Zealand dining behaviours. It was concluded during the early stages of literature research into New Zealand culinary traditions hat dinner was he subject for the case sulies and pariciper observation. Dinner can also be identified as the most opportune in extending academic commentary on contemporary dining experience. The participant observation was framed on sharing a meal within the four homes of willing participatory household of which a typical meal would take place, offering locality within the home basic meal structures, household member roles, archetypes $\&$ applinces used, and of course any interaction with digital tech and its role while dining. Photography was the primary method of data collection throughout the course of the meal where emphasise was placed on participant interaction with spaces in the home, ital tech.

Immediately following the participatory meal semi-structured interview took place. Framed more as a casual discussion, the semi-structured interview provided participants with a prompted discussion on their meal preparation and dining patterns of behaviour, the basic archetypes and appliances mos commonly interacted with, meal structures and and identifying their own digital tech etipere home and around dining Using an interview guide afforded the prompting questions a structure while creating a casual discussion provided opportunity for participants to discuss and elaborate freely on areas of their choosing. This effectively allowed for critically assessment on aspects they instinctively did were voice recoded to sense the dischion flows waturally without following a formalised secuence that other recording techniques would create, such as written recording. The recordings were
hen transcribed using the intelligent transcribing
Published in 1913 as a provocative study into thentury America, Christine Fredericks "The New Housekeeping" employs movement coping a vistul tool to validate hor own belief in

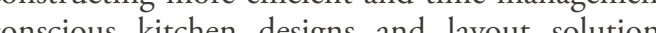
The adoption of movement mapping as a research method has afforded a secondary visual supplement to the research: a minimal, stylised floor plan of he houschold was initially defined inclusive of movenent dital technology was indicated. The plan as a dotted line path. A circular symbol is used to represent the specific location where the articipants most commonly dine.

The final stage of the case study analysed the four household personas and presented them collectively in a sequential format. From this dat Generation Household, Generation X Household Household, and Silent Generatio Household. These personas each represent their respective generation-based demographic in regard

Jean-Claude Kaufmann's 'The Meaning of Cooking a household's patterns of behaviours and their (Kaufmann, 2010). Additionally, the person method is of interest in order to differentiate and across all for houshold wes. This the supports the intentions of the research in considering the impacts of technology on the New Zealand domestic dining experience. the reforming of the housewive's kitchen in early dentifying relevant rooms and spaces adjacent to all appon andiving areas. Within this floor plan were then interpreted and ploted within the floo has also inspired the adaptation of person own analytical thinking towards these pattern present target demographics individually, allowing 
Concept sketching was the first research method practiced in the development of the design outcome: needs and desires of Kiwi households. This method afforded the ability to document and critically assess the conceptual ideation and its evolution throughout the course of this twelve-month research project. Concept sketching is an integral application for all designers, having been introduced to it at high school level it is critical when actively engaging in which it has been utilised is hand sketching on paper with drafting pencils and pens, and digitally via computer aided design (CAD) software. The sketching context has varied at all stages throughou the research commonly composing visual mind mapping, basic concept and form ideation an manufacture exploration, and material identificatio and exploration. Concept sketching has constructed a strong basis of which supplementary method can be applied in forming a concise concept research stage, particularly as a means to inform the aforementioned value of material prototype development.

Sketch modelling was the second methodolog material prototypes, in again following an iterative esign process. Sketch modelling was used to critically assess the ideas formulated as a result of the concept sketching process, as well as the ideas and observations collected from the case study research. Like the concept sketches, sketch modelling aims to The models produced bave the benefit of entive user testing and the collection of user feedback. As a result of the feedback elicited two design iterations were selected for further development. More specifically, these two design iterations were selected for their contrasting integration of both digital and tangible interface opportunities. Throughout this stage sketch modelling was
continually engaged in as a means to refine the two concepts in to a singular, final design. Ultimately the collective utilisation of these methods provides the opportunity to critically assess the iterative development of the design through to the experience prototyping stage.

Note: Although the digital interface is presented in a static form throughout the prototypes, each iteration supported a series of wireframe mock-ups of the proposed digital interface. The generation of these wireframes were iterative in themselves, and the design was developed to reflect a consistent

Experience prototyping was the final method utilised in identifyin prototyping aims to identify and evaluate the successful and unsuccessful elements of a concep through interactive engagement with prospective users. In the context of this research project the users' included case study participants. As a result of this process a third, more refined prototype prototyped desion more clearly ression of the proposed digital interaction display materiality the design details. By offering participants the chance to interact with this refined version of the design, they were better able to provide critical commentary on the tray's tactile elements. Of particular concer was the tangible interface and overall scale of the Smart Tray prototype where the physical grasping multiple particants allowed for clear and concise critical feedback. Upon completing this stage the participant commentary was assessed and implemented within considerations that lead to the further refinement and production of the fina designed object.
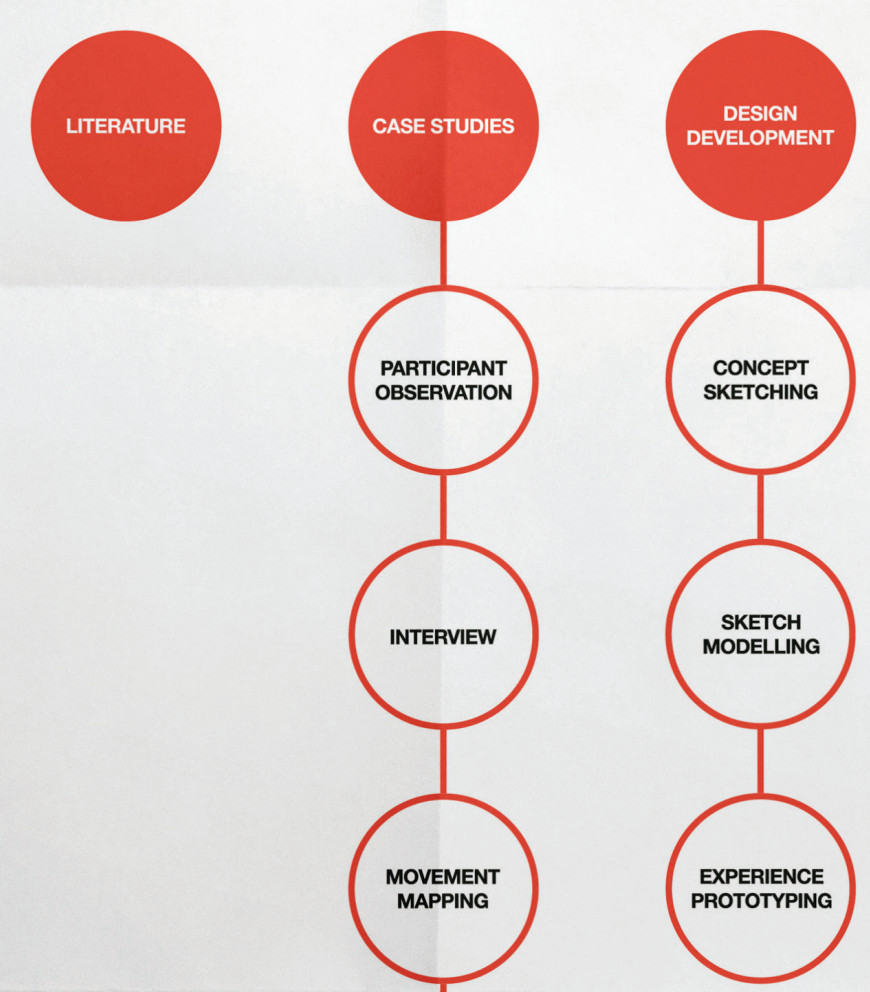
CASE STUDY

As indicated in the section titled 'Research Methodologies', four personas were defined as a
means to structure the case studies: Millenninl means to structure the case studies: Millennial Generation Household, Generation X Household, Baby Boomers Household, and Silent Generation
Household. These personas each represent their Household. These personas each represent their
respective generation-based demographic in regards to meal preparation, dining, and interaction with technology. The case studies that follow are sequentially representative of these personas. The Millennial Generation is therefore presented first, with the Silent Generation household concluding the case studies. It is important to recognise
that independent, adult New Zealand residents collectively form the general target demographic for this research. 
49

CST

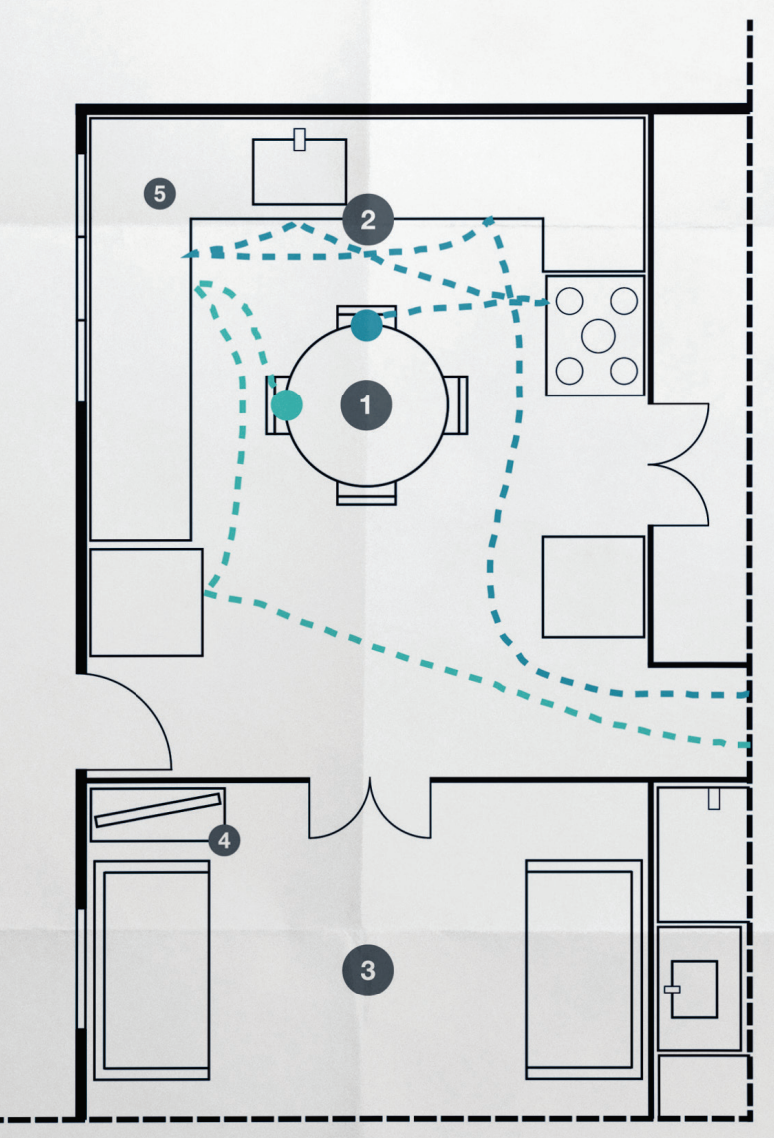

@

CASE STUDY ONE

1900S

PARTICIPANT ONE

19
BARISTA

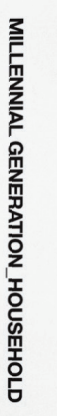

Case Study One

MILLENNIAL GENERATION

$1980-2000$

ABOVE: Figure 23. Participant movement map within dining space of

the Millennial Generation household. 


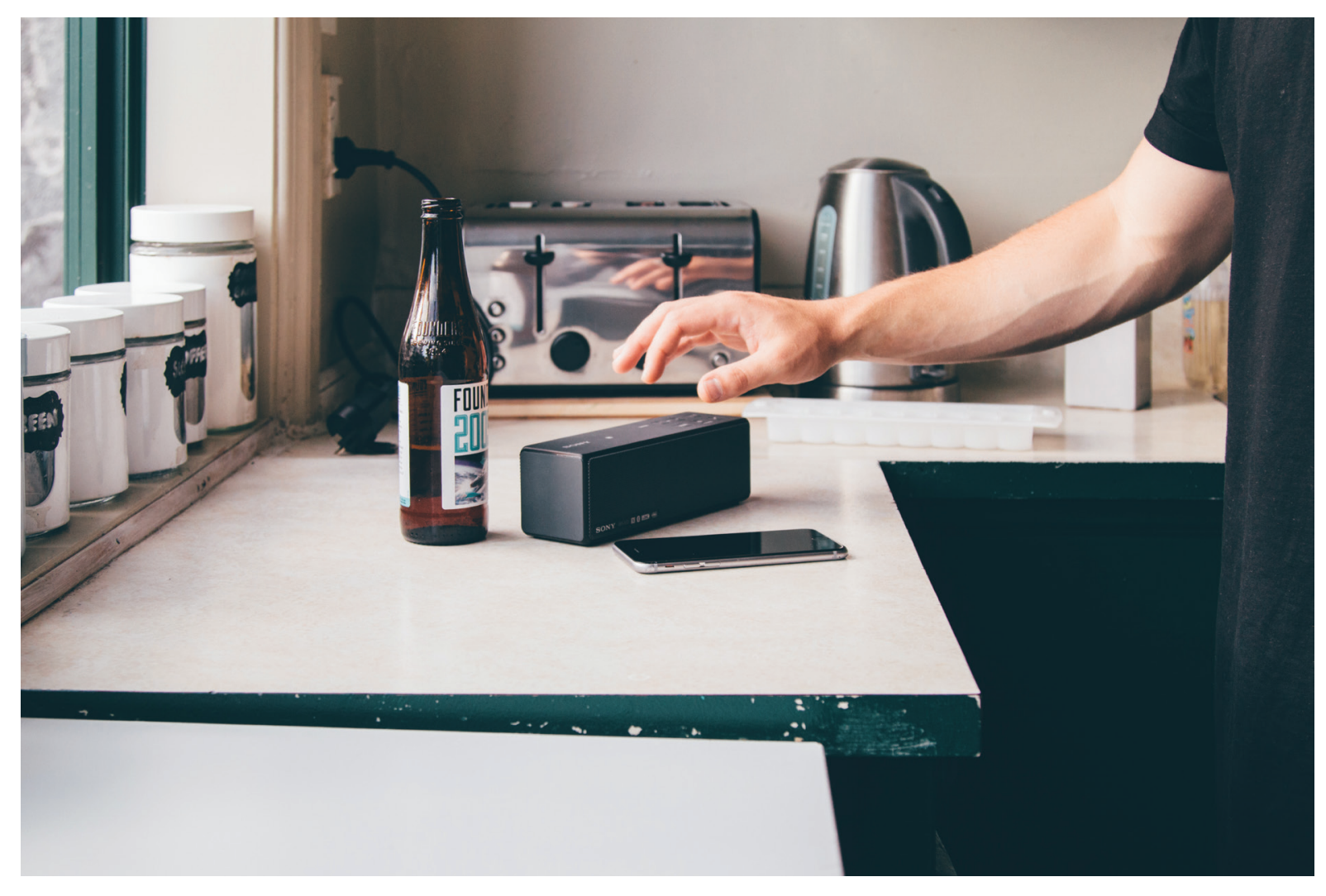

The term Millennial Generation in most academic to the early 2000s (Strauss \& Howe, 2000). This generation is credited to be the first to grow up with the Internet, being the most exposed to digita technologies since birth. They are also thought to generically contain a strong sense of community

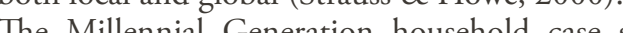
The Millo of Newtown. Built in the early 1900s the three-bed is home to Participant One (P1) and Participant Two (P2). P1 is a nineteen-year-old female currently working as a Barista and partner $\mathrm{P} 2$ is a twenty-oneyear-old male student.

Kitchen

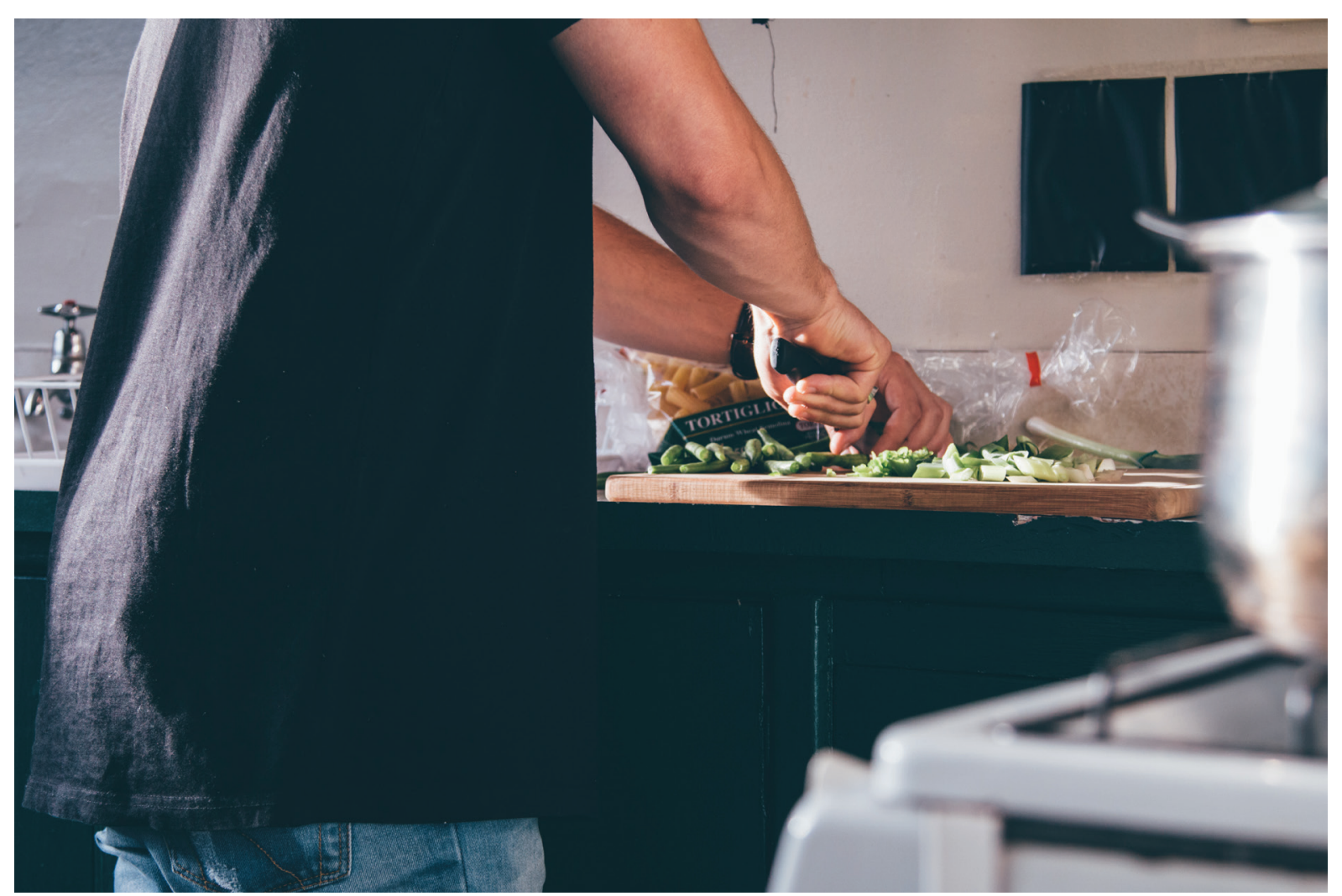

Collectively $\mathrm{P} 1$ and $\mathrm{P} 2$ admitted their kitchen is part of a stereotypically dated student flat however they embrace the space as a central hub to their living. P1 reflected on a space of social congregation and a lot of laughter as "everything together happen here." A round dining table takes pride of place in the $A$ tich incredibly functional kitchen where strobs was placed on the traditional use of the dining table - being seated for a meal together and delving into free flowing conversation. An interesting observation came from both Pl and P2's desire to eat from warm plates as an important aspect to their evening meals.

Dining When patterns of behaviour around dining are
concerned P2 affirmed, "we don't fluff round - we normally just eat a main dish and that's it." They both identified a culinary tradition of white or red meat and vegetables, cooking American and British Aspred dishes why cexplong Med daily basis, expressing their desire to eat fresh as often as possible. This then flows on to the lack of storage ods fresh. Because they cook their own meals they feel a responsibility to sit down formally and enjoy them together, where they reflect takeaway meals would often result in being eaten on the conch as a more casual meal having the traditiol use of a dining table I observed a fairly relaxed and adaptable dining culture, where passion for good food, and intimate bonding and conversation was evident. In this in was also complete integration of kitchen and dining space, which seemed to streamline commo the

$$
\text { Tech Etiquette }
$$

Joining P1 and P2 for dinner I observed P2 interact frequently throughout the night with Bluetooth speaker system connected wirelessly to his smartphone. This provided a ubiquitously ambien soundrack, somenting he belined was exable sitting down for their evening eal. This seemed to subdue the formalities of eating at the dining table as well as prolonging the overall mealtime period as background music made pauses in conversatio feel more like intermissions rather than signs to end the conversation and thus the meal. Although both participants were rased in traditional settings on to the dining "occasionally I find myself reaching for my phone to check it." This explicitly presented both participants acknowledgment of their use of digital tech and its value to enhance a setting, whilst also recognising the need for minimal interactions with it during 

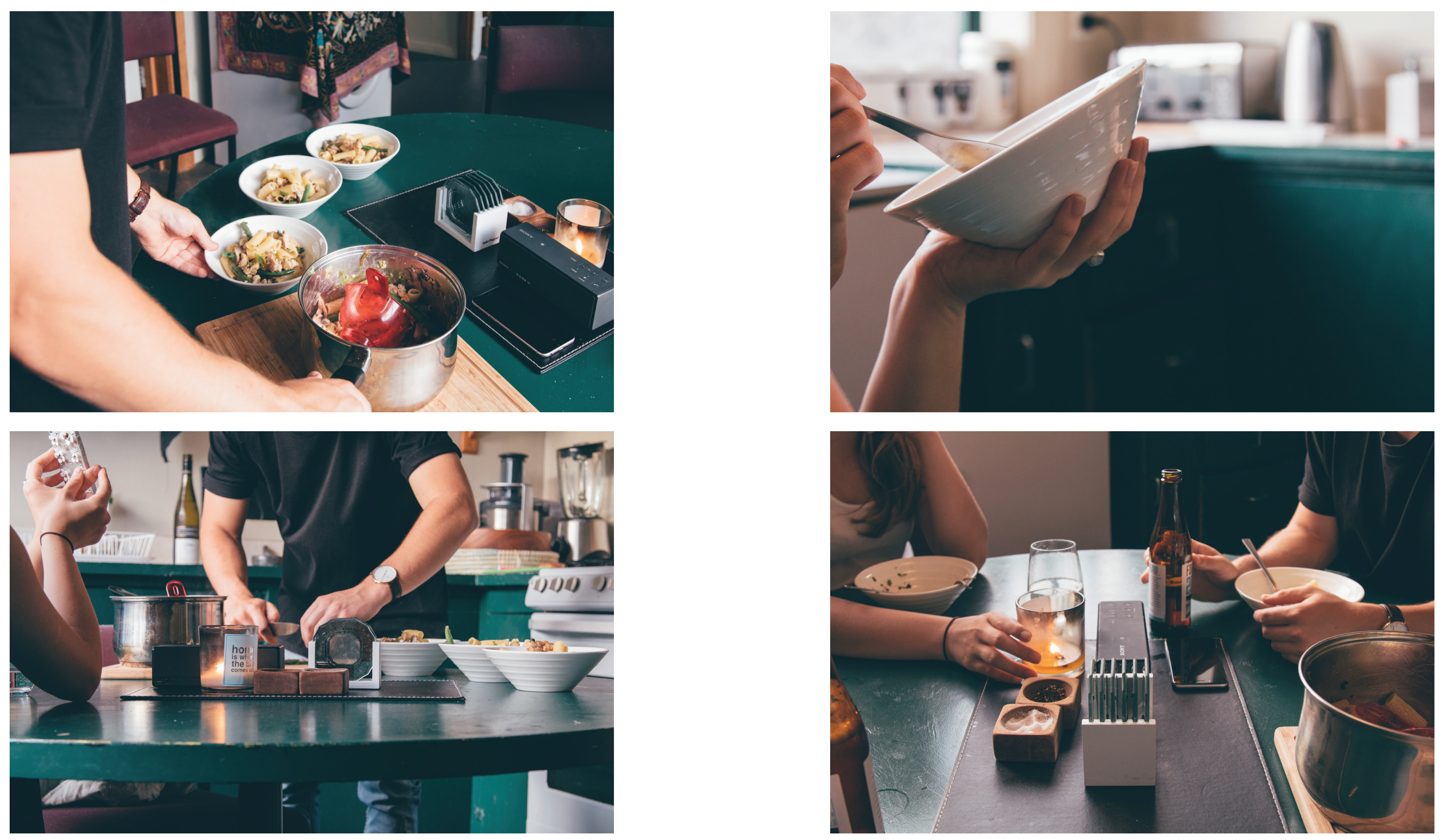

TOP: Figure 25. CS1 often serve a singular dish in producing a meal 
cse

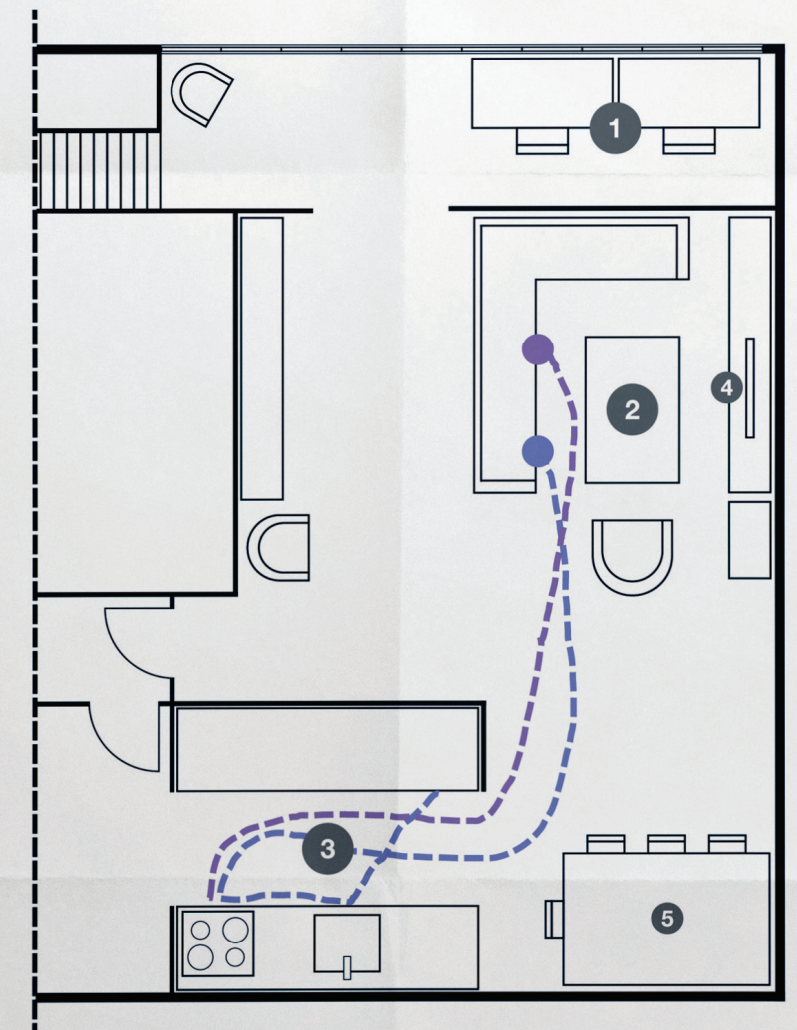

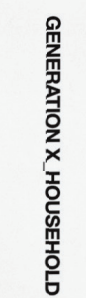

\section{Case Study Two}

GENERATION X

$1960-1980$

$\rho_{-}$

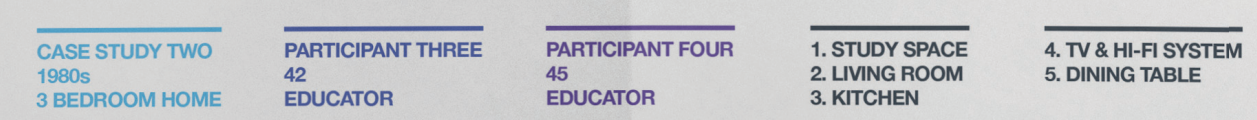




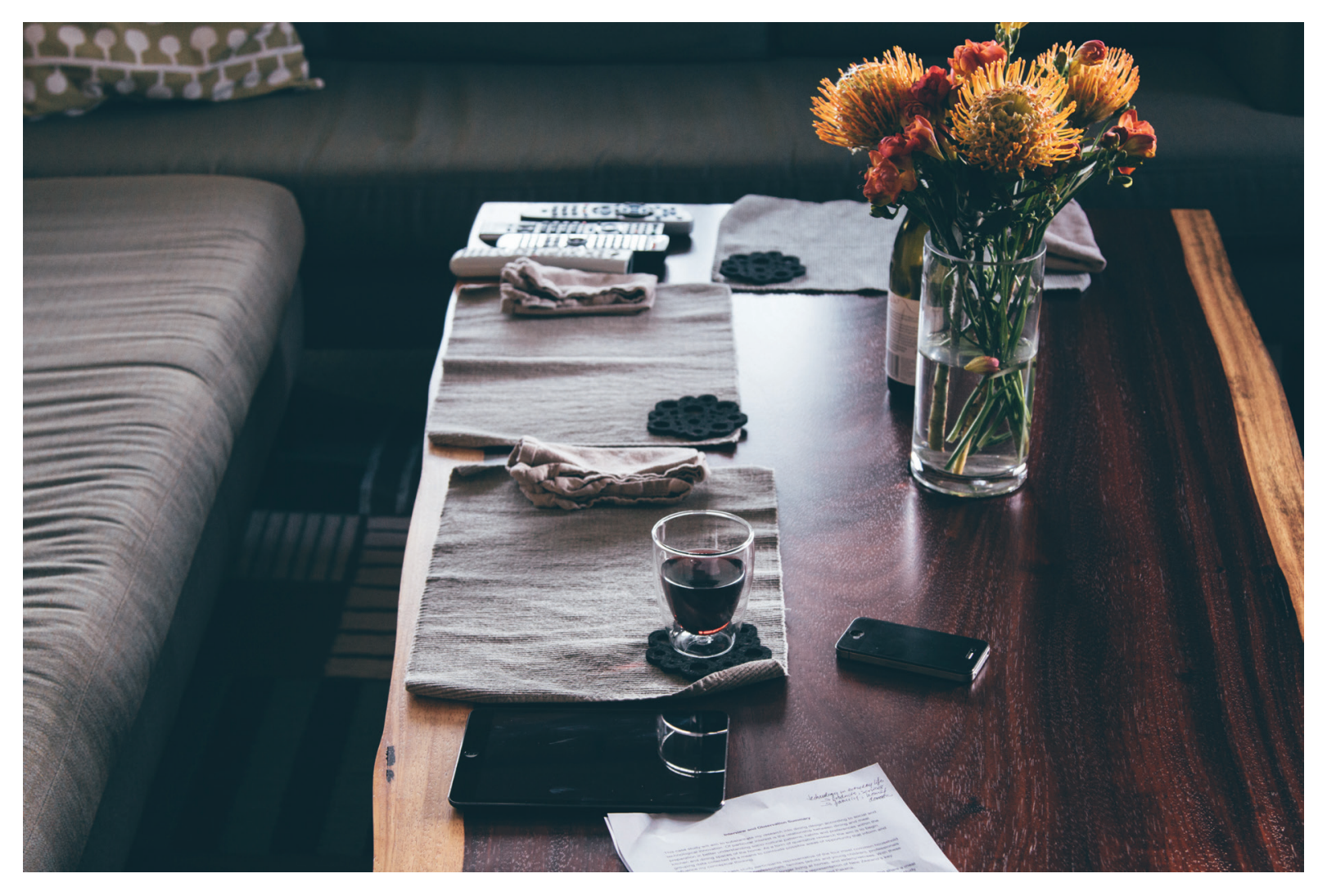

Generation X commonly refers to the birth years ranging from the early 1960s to the early 1980 s with the generation it precedes represents a more heterogeneous generation, embracing social diversity of characteristics that include race, class, religion, ethnicity, gender ident

(Generation X, 2008). Placed on the slops of Wellington's Island Bay, framing views of the Cook Strait, the Generation Lockwood home. Its occupants, both tertiny educators, are Participant Three (P3), a forty-twoyear-old female, and Participant Four (P4), a fortyfive-year-old male.

\section{Kitche} Similar to the Millennial household P3 and P4 own home. For this reason they both feel they are in period of tolerance with $\mathrm{P} 3$ describing the kitchen as definitely not the heart of the home $[\ldots]$ regardles of its size I feel the kitchen isn't a place to hang out or socialise." P3 and P4 actively cook together
when preparing dinner most nights, and alluded to wheir praptive use of a small kitchen simultaneously. Both choice and dietary requirements dictate the dominant purchasing of fresh foods on a daily bas, depending on the fridge to keep foods fresh.

\section{Dining}

Interestingly P3 believed culinary traditions "are becoming more singular thanks to international salads and fish. All meals are relatively simple, speaking to their daily patterns where dining, P4 believes, is completely convenience and comfort ofTV and an emphasi is placed on dining togethet Even if this results in no talking where an intimate experience takes place through the simple pleasure of enjoying each other's, or a guests company. Admittedly P3 and P4 do not like the idea of eating at the dining table as it is not comfortable in their is total honesty as "what we really want to be doing siting fact I observed the dining table being used as more of a kitchen space utility, perhaps a role of the future?

\section{Tech Etiquette}

In extending on the adoption of the sofa and living pace as the place of preferred dining P4 revealed or a movie while eating as it is not scheduled and remains entirely in our control, fitting with when we weel like eating." T whe digital tech devices such as a TV, hi-fi system, wath pint did he dig purly on convesan. At no pist case sud I was exposed tor. Sint or to the throughout the night. Although played through a more substantial hi-fi system again I observed the utilisation of wireless Bluetooth control as $\mathrm{P} 6$ seamlessly changed tracks while mid conversation. However P3 and P4 outlined a general rule aroun the exemption of calls or online conversation with mealtime of pervasive tech integration.

TOP: Figure 28. CS2 embrace an open tech etiquette where multiple devices were observed, and interacted with throughout the study. scratch, where formal place settings restect this passion for goo 

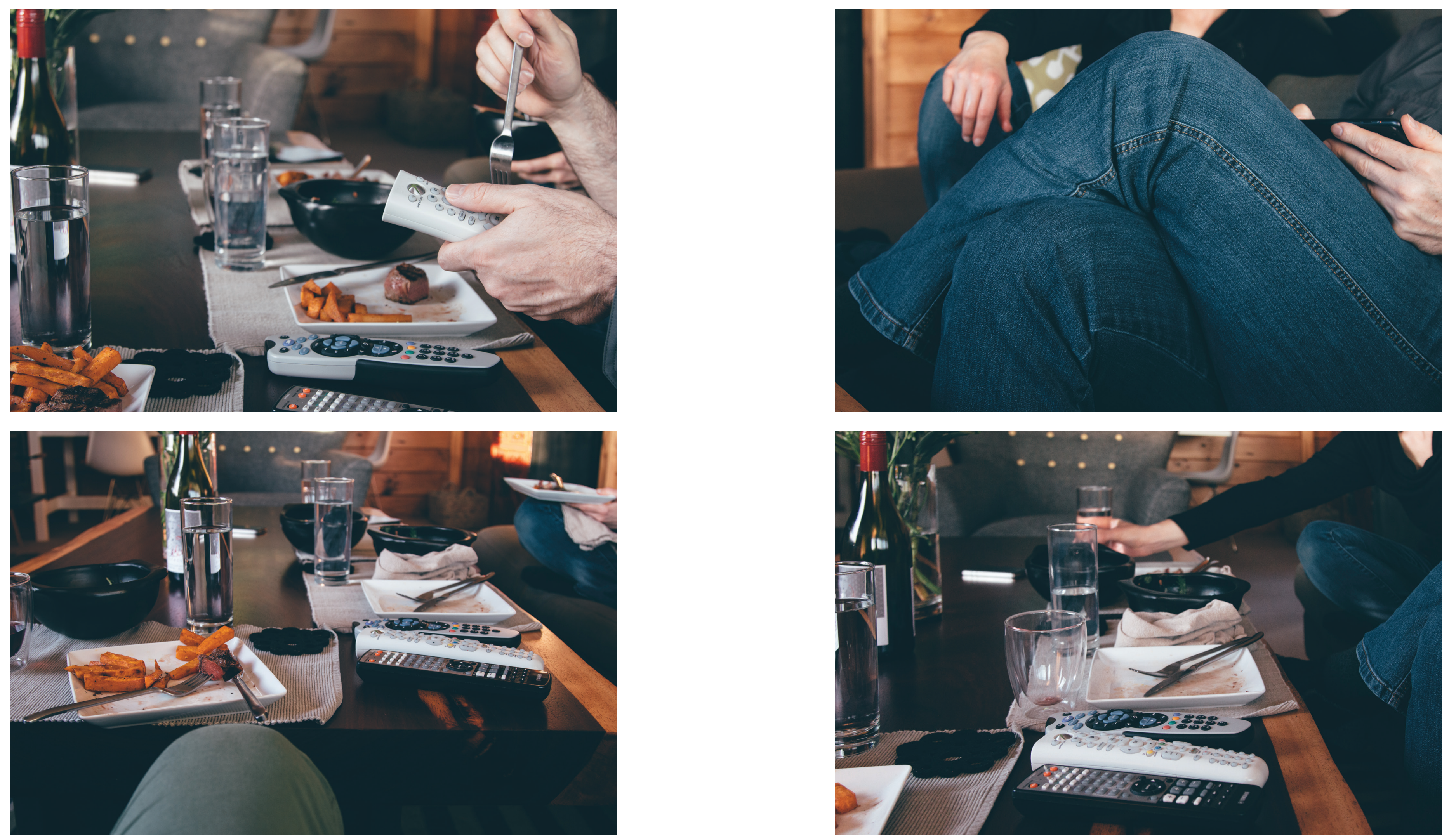

TOP: Figure 30. Dining in front of TV enjoying streamed TV shows reflects CS2's emphasis on connenience and comfort.
BOTTOM: Figure 31 . CS2's cluttered dining place setting visually communicates opportunity to better integrate dining and digital

TOP: Figure 32. Observation of both P3 \& P4 interaction with table BOTTOM: Figure 33 . CS2 enioy a casual dining exparienco purposes
BOt digital technology is integrated in enhancing the experience. 


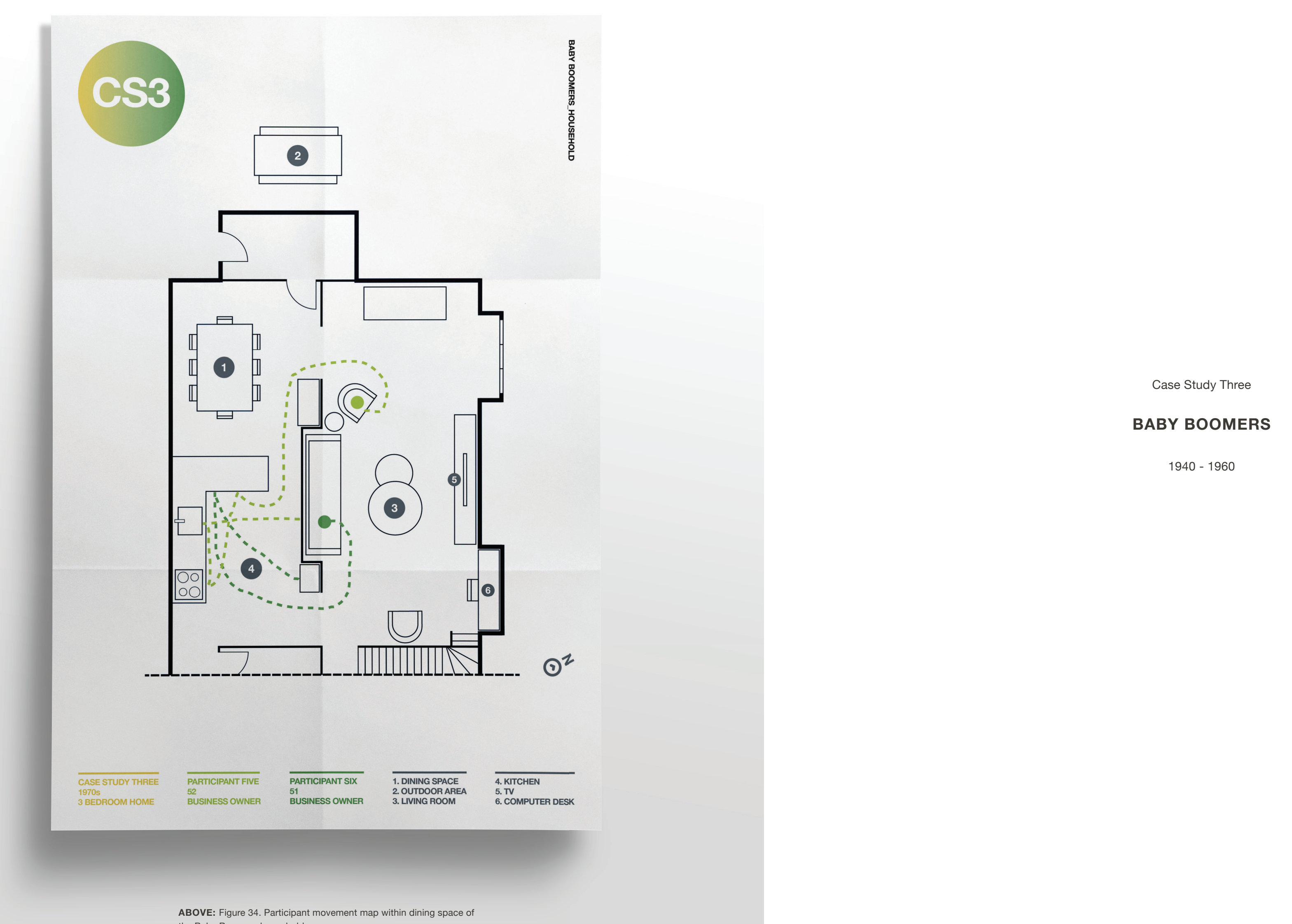




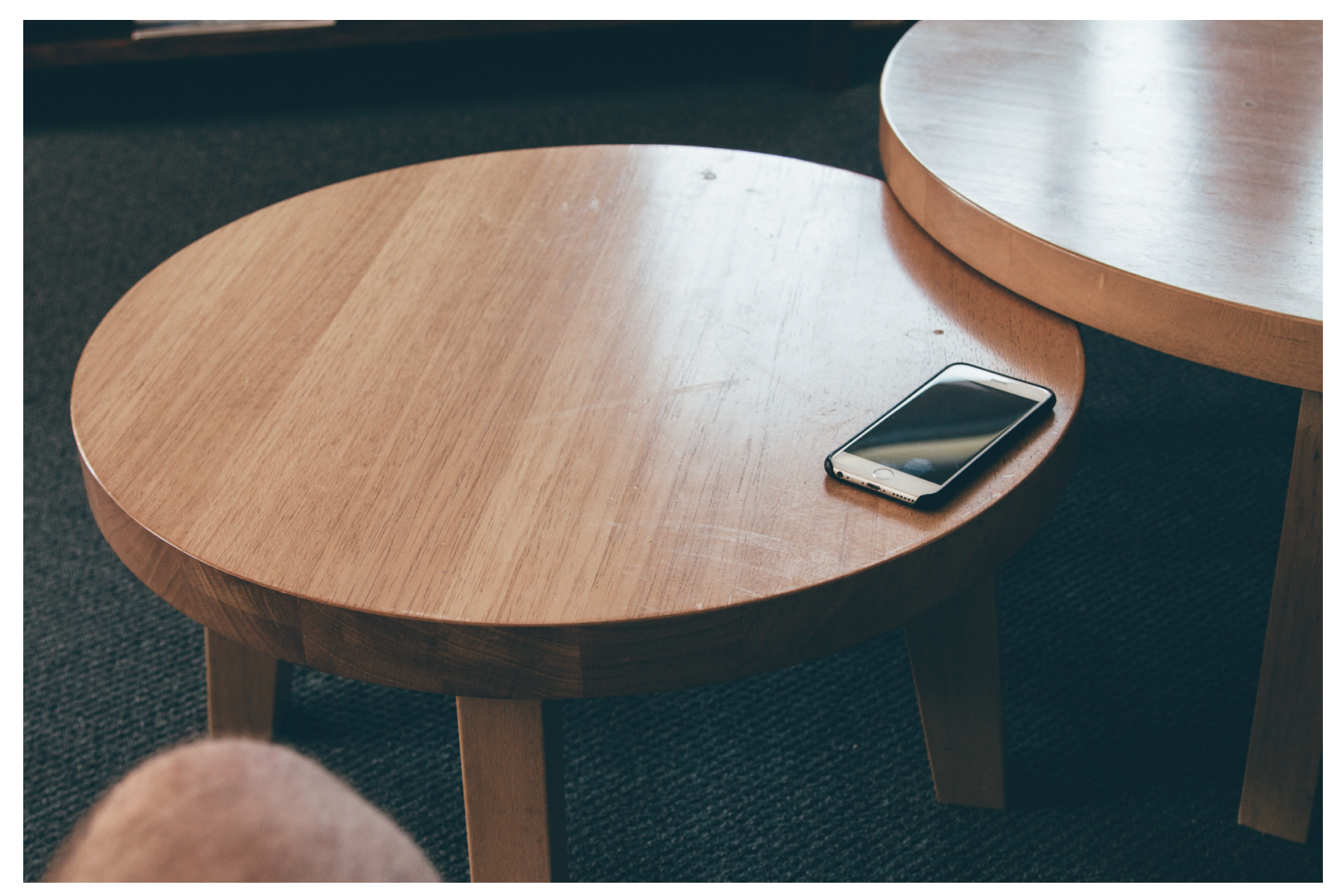

The Baby Boomers generation represents those born within the early $1940 s$ and $1960 s$ during the postWorld War Two birth spike. They are commonly associated with bis the sef Grabidies in post-war housing and eduction (Werner, 2011).

Participant Five (P5), a fifty-two-year-old female, and Participant Six (P6), a fifty-one-year-old male, are a self-employed married couple running their own business. They share a three-bed home in the Normandale hills of western Lower Hutt, a

$$
\text { Kitchen }
$$

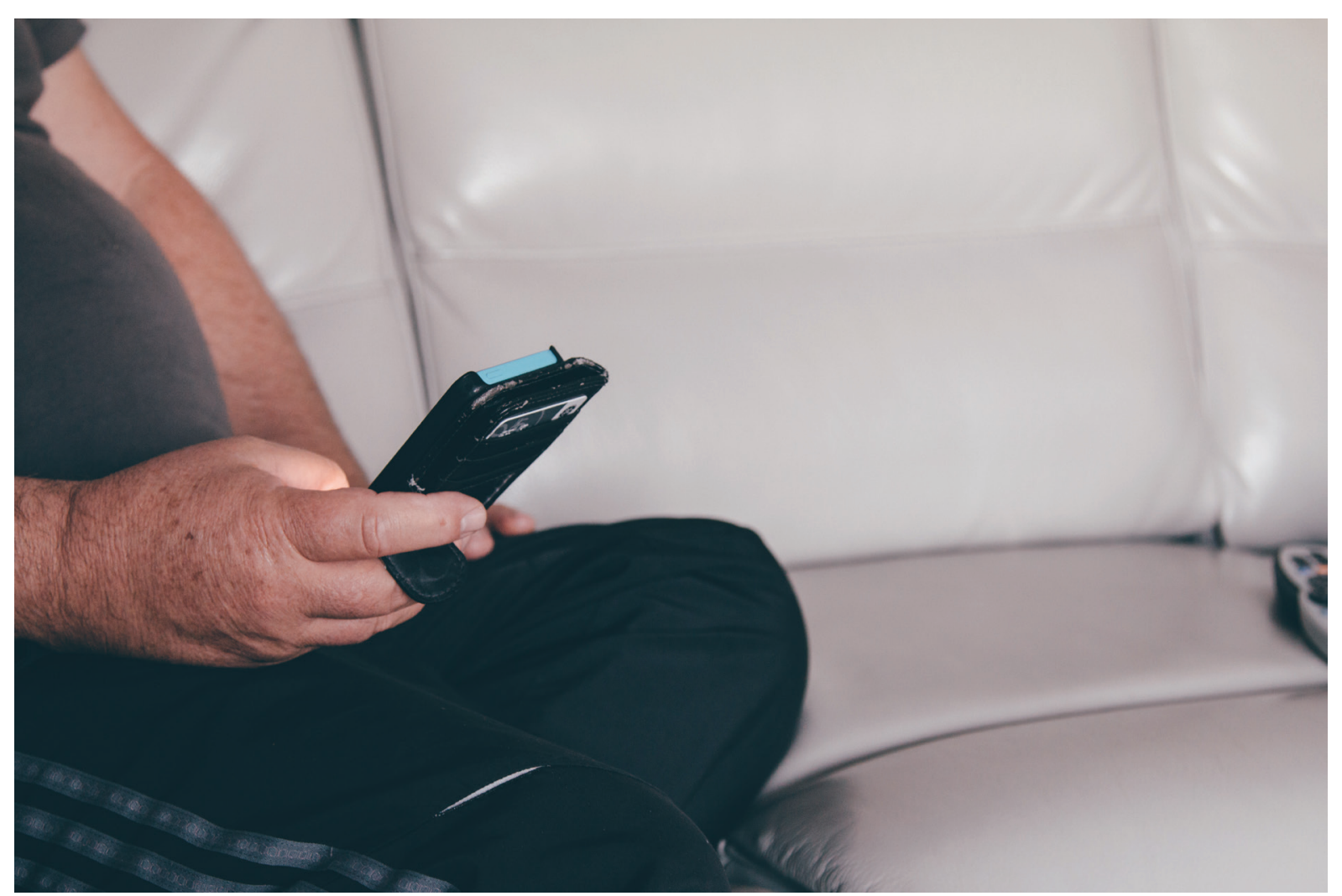

P5 and P6 believe their kitchen is a logical space acting as a practical thoroughfare in connecting living spaces with other parts of the home. For this reason both participants see the living space, which . time despite its comfortable size as P5 reveand "we generally split roles each night for dinner - one person will do the cooking while the second does the washing up." Due to their busy schedules food tends to be purchased on a weekly or half-weekly basis. For this reason they depend heavily on the freezing of their meat, vegetables and bread in particular It is interesting to note that out of all fou housenold case studies the Baby Boomer's relied most heavily on their pantry and fridge-freezer storage space.

Dining

P6 describes their culinary tradition as "typically western-kiwi. We have pretty traditional mea while also bing partial to pas wond recognise fry dishes." They also revealed the meals are very commercialised, coming from popular culture outlets such as magazine's and TV cooking shows. P5 also interestingly admitted to their ritualised "tea with the six oclock news as an important structuring to their day, acting as an "indicator that the wal and have a casul convers tine to enjoy debate in front of the TV:"

Although P6 described both participants as not the most "tech savvy they do not see a major problem

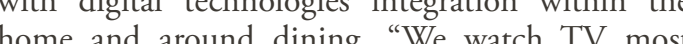
dinners, so I find tech aro "We wh fine" concluded P5. Throughout the meal I observed constant interaction between the TV remote and P6 while both shared a constant focus on the TV and each other during conversation. $\mathrm{P} 6$ also believes taking a phone call is fine as mealtime is a casua occasion and being connected to the rest of the "world seens heng stacing, "dinner together is made or received then so be it it is $j$ ust the world we live in now. The same way I would interrupt dinner for the bathroom or pull the curtains for example." 

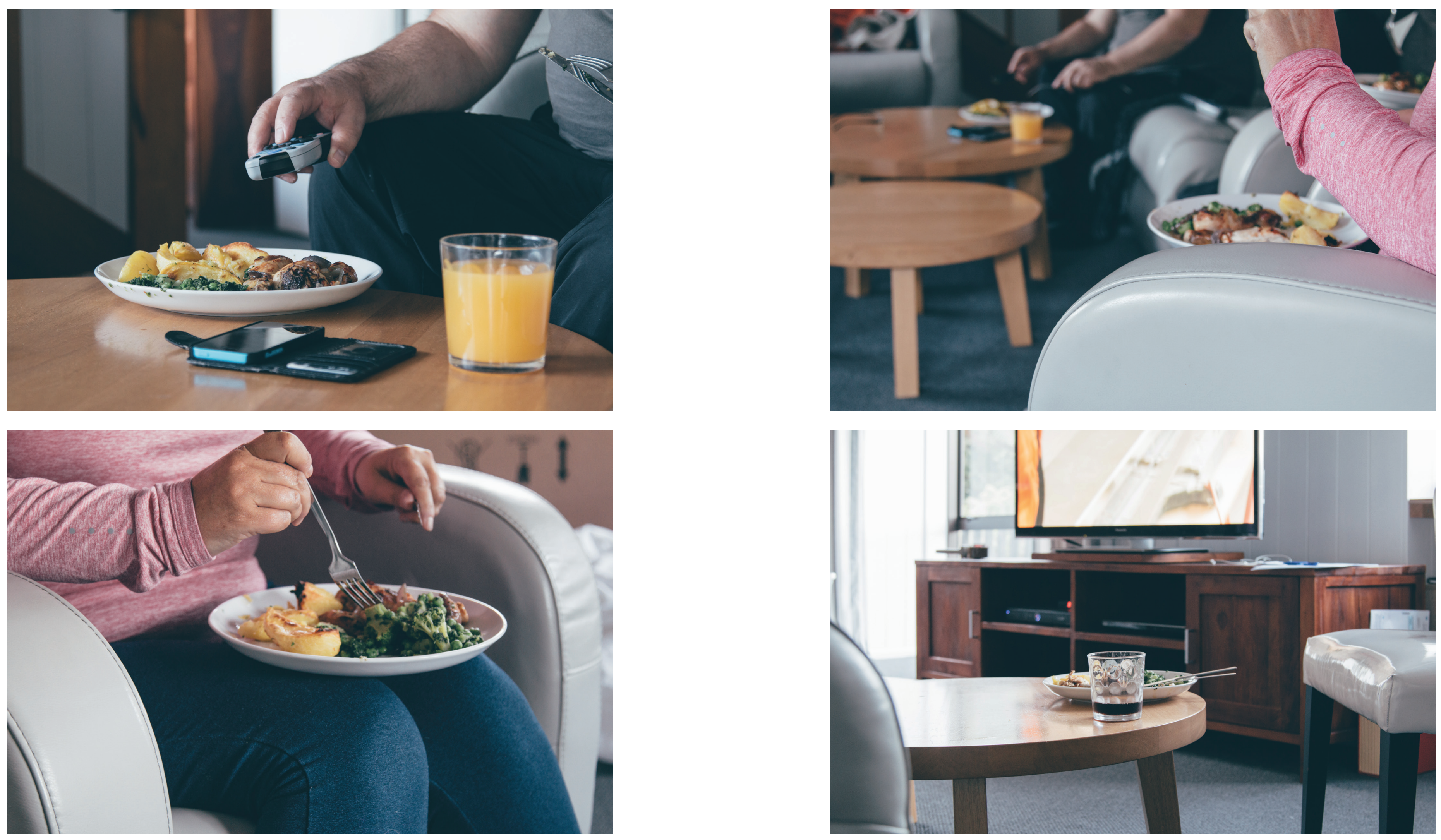

TOP: Figure 37. CS3 dinner strucutre centred on dining in front of the 
CS4

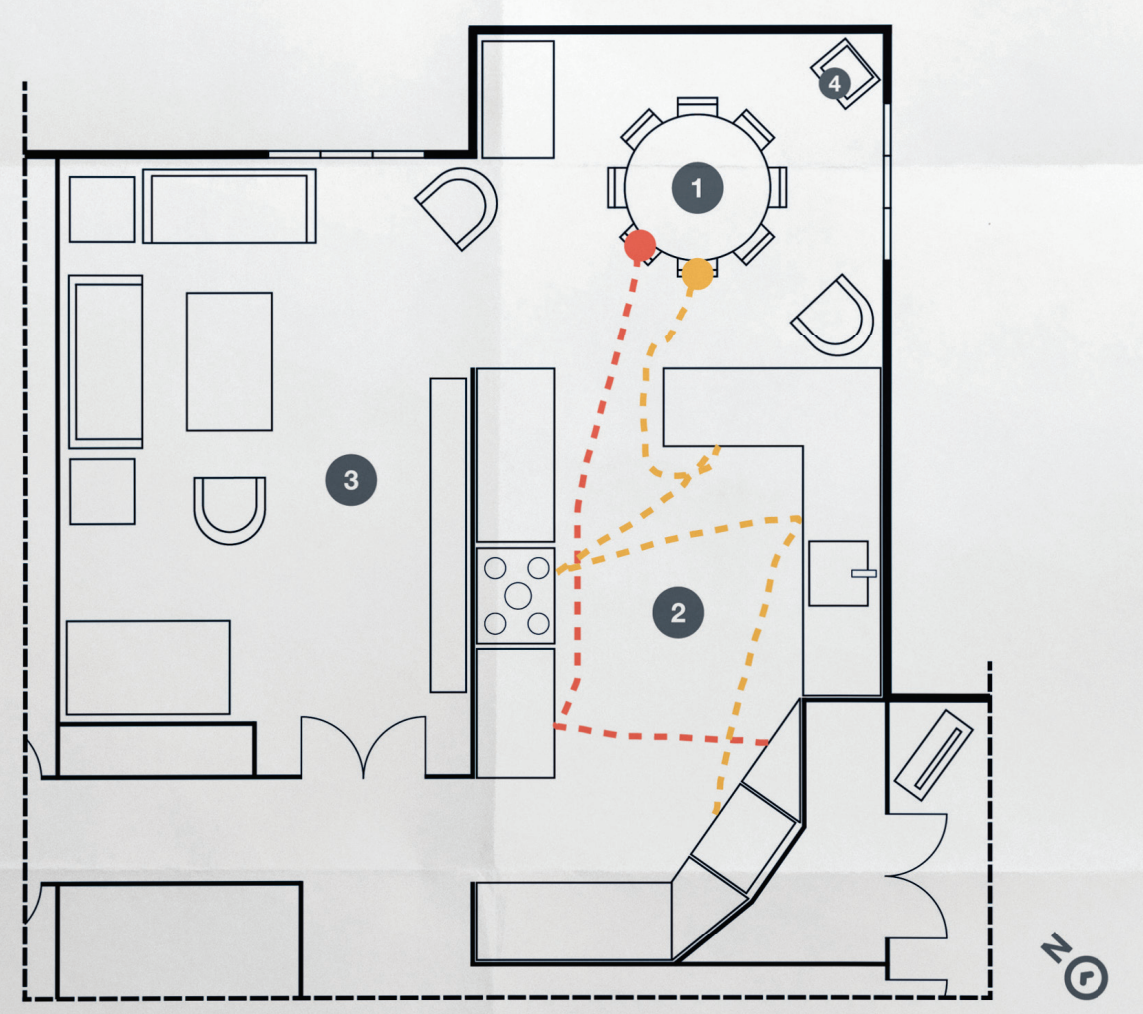

Case Study Four

SILENT GENERATION

$1920-1940$ 

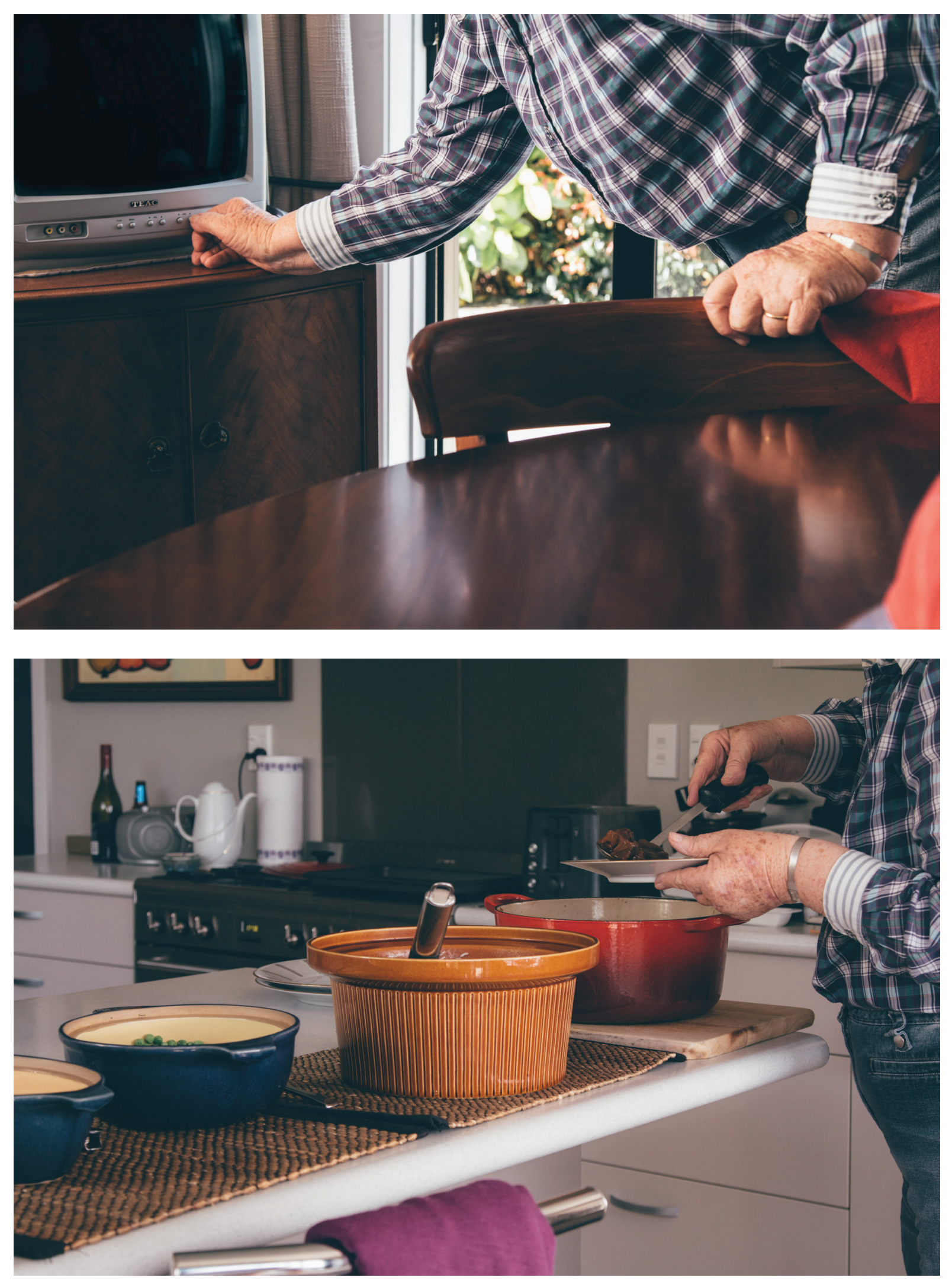

The Silent Generation commonly represents those born between the early 1920 s and 1940 s, having
been born within a period of relative economical been born within a period of relative economical
insecurity causing low birth rates, reflected in the ltimate action of two World Wars either side of the generation's timeframe (Carlson, 2008).

The fourth and final case study took place in Aote with Silent Generation participants, eighty-threeyear-old female Participant Seven (P7), and eightysix-year-old male Participant Eight (P8). Bot retirees now enjoy stunning views of Tithai Bay and Mana Island in a three-bedrom

Kitchen

Boasting the youngest home throughout this case study research I observed a relatively modern and incredibly well maintained kitchen and adjacent open-planned living space. While $P 7$ does all

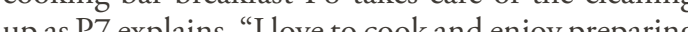
amost everything from scratch. I roly on regilgr visits to the shops to provide fresh food to cook and the fridge is essential in keeping things fresh." The kitchen is an incredibly spacious size where assorted kitchen utensils and books indicated the kitchens, and cookings signific

Dining

P7 indicated that their collective culinary traditions both reflect the foods they were served as kids by
their parents. In both cases this included Irish,
Scottish and English elements. P8 described that mos peals are fanly traditional served on oven-warmed

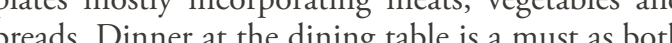
peas. Dince at hie ding table is a mist as booh politeness and rood standans. With multiple allows everyone to feel involved. Similar to the Baby Boomers participants P7 and P8 always structure their dinner around the six oc clock news with a small TV placed strategically next to the dining table. P7 explained, III start cooking at 5.30pm with the intention of "Inving dinner just as both never eat separately as they feel togetherness is crucial aspect to their lives. P8 revealed "despite using a dining table watching the news reflects the casual nature of our meals. This is not to say we dont communcate, rather in fact the opposite as it perdes counless reason for conversation and even

\section{Tech Etiquette}

P7 and P8 appear to live a simple life with regard to their tech etiquette as they choose to limit its use to the $\mathrm{TV}$, radio, phone line, and sporadically brief interactions with email and the Internet. Considering the integration of tech and dining denying any sugestion of using the, promptly denying any suggestion of using the phone or
Internet while dining.for the bathroom or pull the curtains for example." 

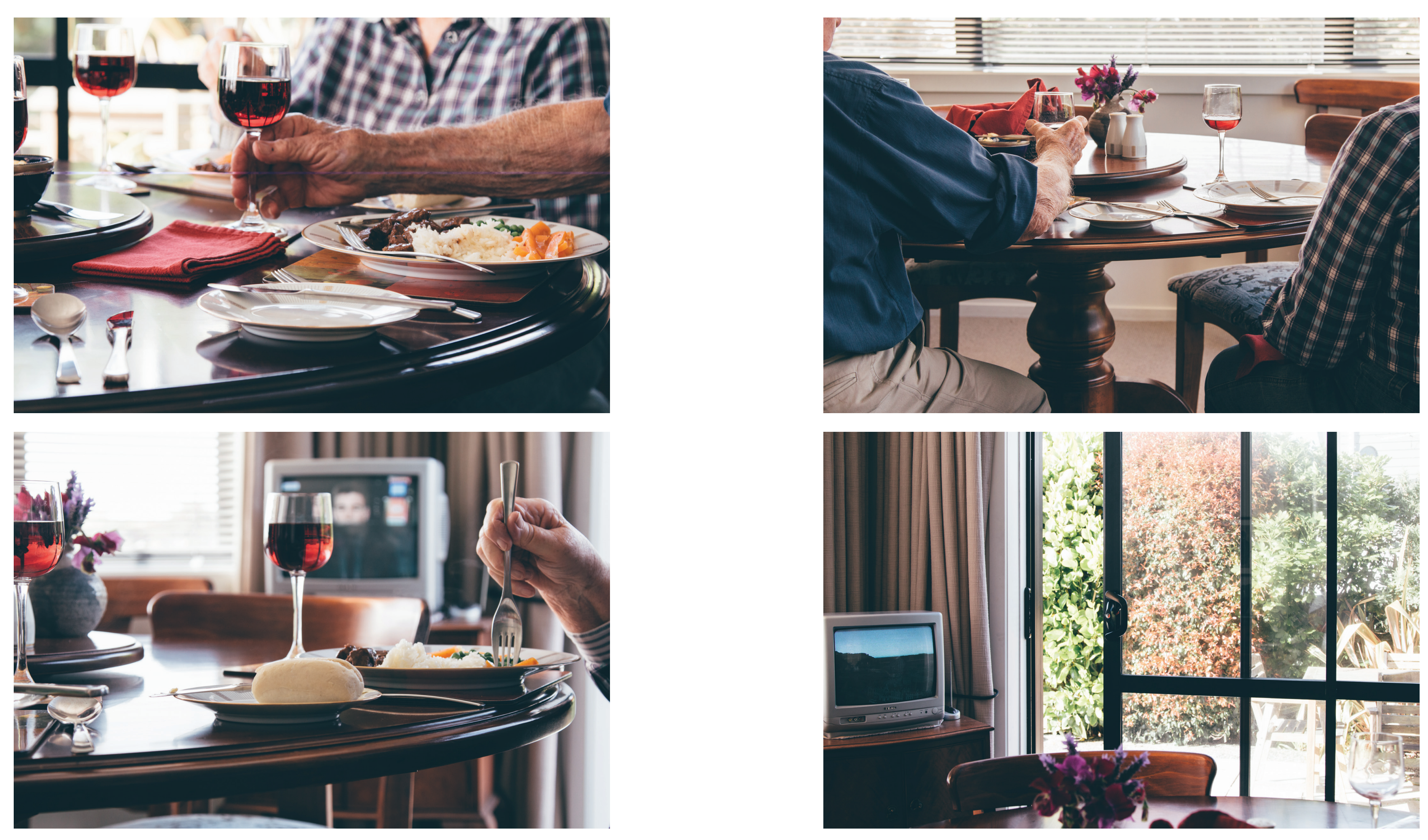

TOP: Figure 44. Dining together P7 \& P8 embrace a traditional dining
experience and utilisation of a dining table and formal place setting.

BОтTOM: Figure 45. Dining in froct of a

TOP: Figure 46. P7 \& P8 both believe the dining table maintains
appropriate social connection and politeness whether together

BOTTOM: Figure 47. A small TV expresses the value CS4 place on 
HOUSEHOL__SUMMARY

\begin{tabular}{l|l} 
KITCHEN DINING TECHETRQUETTE &
\end{tabular}

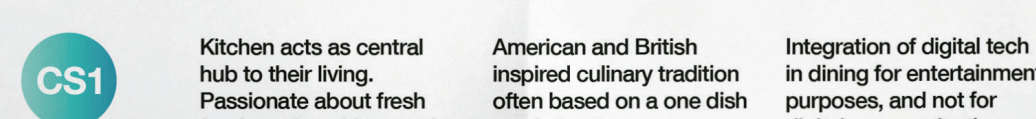

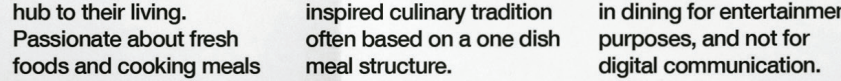

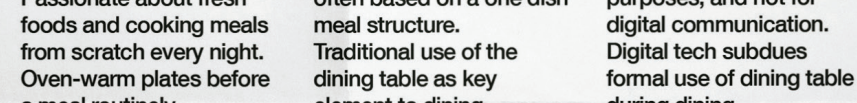

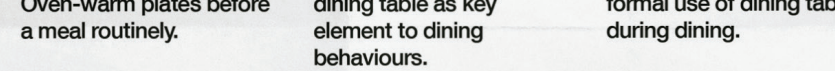

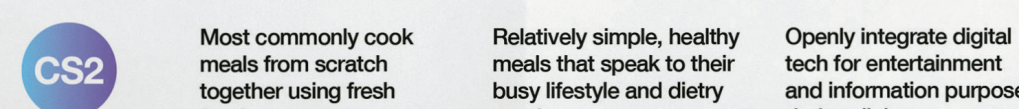

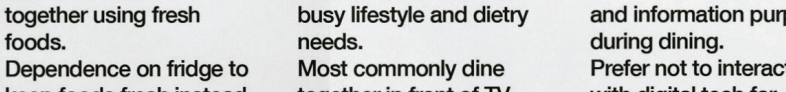

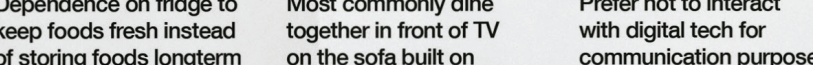
$\begin{array}{lll}\begin{array}{l}\text { of storing foods longterm } \\ \text { in pantry. }\end{array} & \begin{array}{l}\text { on the sofa buitt on } \\ \text { convenience and comfort. }\end{array} & \begin{array}{l}\text { communinication purposes } \\ \text { while dining as a time to } \\ \text { disconnect }\end{array}\end{array}$

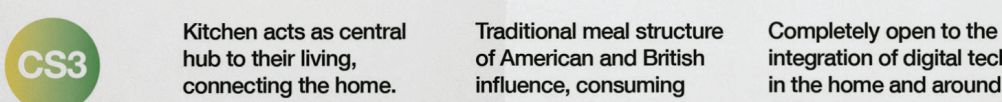

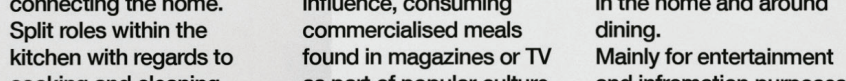

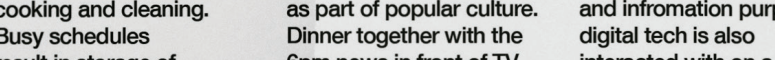

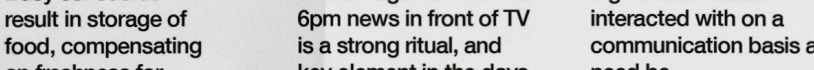

is a strongntual, and
key element in the days communication basis as
need be.

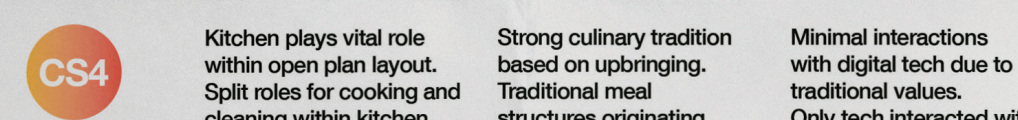

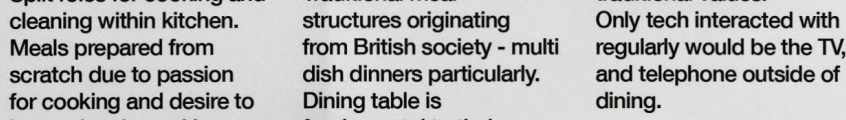
for cooking a and desirine to
invest time in cooking on

Lundamental to their
dining experience.
Dinner is served.

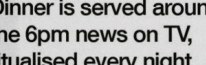

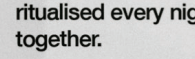

ETHICS APPROVAL

0000022387 Human Ethics Committee
Victoria University of Wellington 
PRELIMINARY DESIGN

The preliminary design stage acknowledges
aforementioned literature and case study research aforementioned literature and case study research
in pursuing an iterative design process. This stage in pursuing an iterative design process. This stage
aims to produce research-led conceptual thinking aims to produce research-led conceptual thinking
that ultimately produces the final design solution for this research project. The design experiments outlined in the following section explore a variety
of research methods in ensuring the final design output accurately addresses the research aims as best possible. 


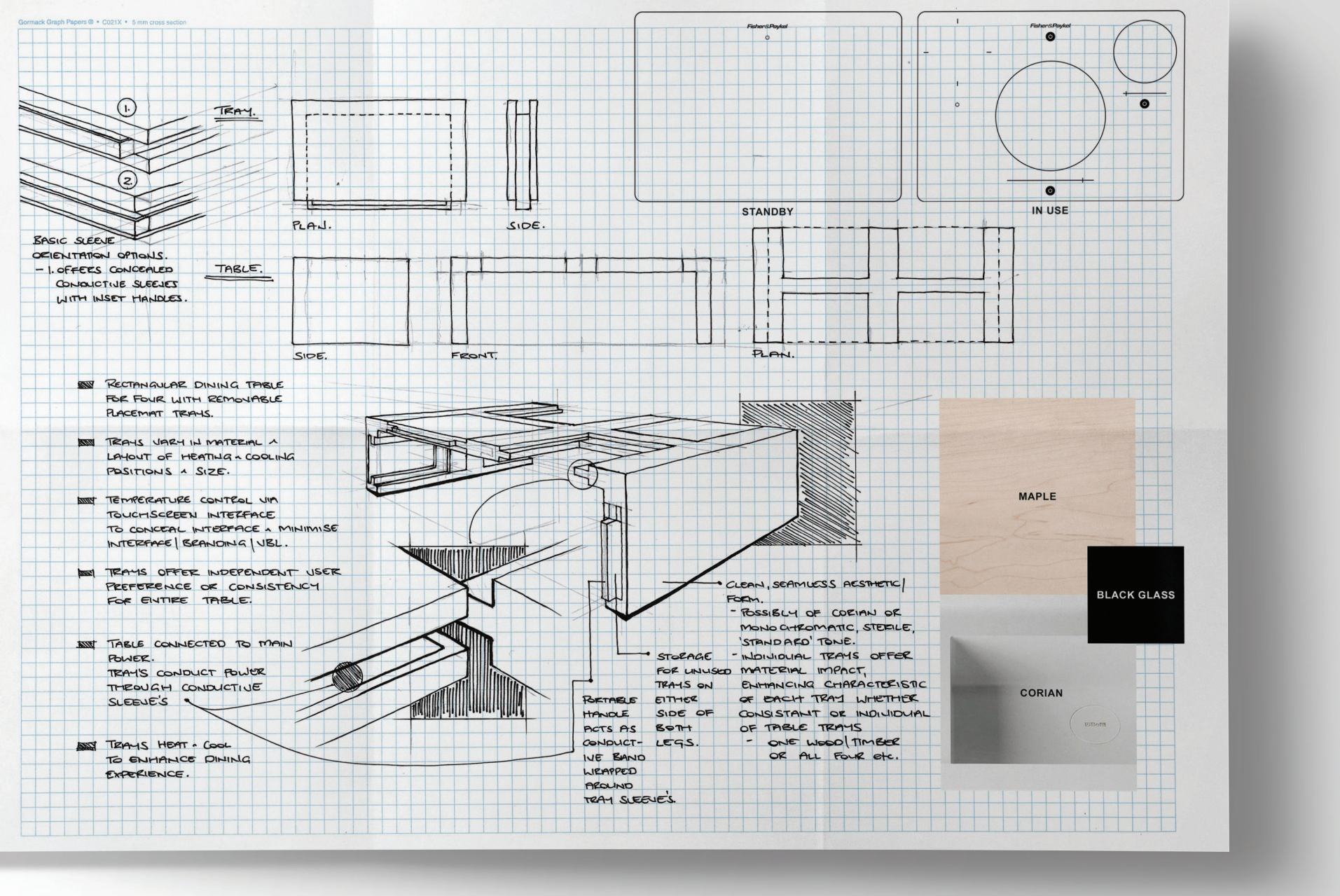

PLACEMAT TABLE CONCEPT

This design experiment aimed to explore pervasive furnishings and products, in this case linking rechnologies integration with both appliance design, functionalities of the kitchen with the basic and the dining table, maintaining the fundamental ergonomic practicality and cultural hierarchy of the speculative appliance device. The concept explores significance that the majoity of the participants four removable placemats within a rectangular placed on the dining table as an important and table with double-sided adaptability. The first valued utility to dining. Despite the fact that all side incorporates a working surface, while the four household case studies described their pattern second houses the appliance device that integrates of dining without the dining table as frequent and induction-heating technology. $\quad$ even, in some cases, preferred, the dining table There exist two predominant purposes for the dining experience. device. First heating and cooling capabilities on the induction surface enhance the both successful and unsuccessful elements. The to provide was most successful in proposing a personal device a portable, adaptive placemat-tray again allows as opposed to a collective table-based device. The the user complete control in where they choose to limitations on user functionality were not successful

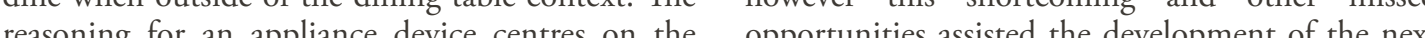
continul development of open plan living spaces, 
Experiment Two

SMART TRAY CONCEPT

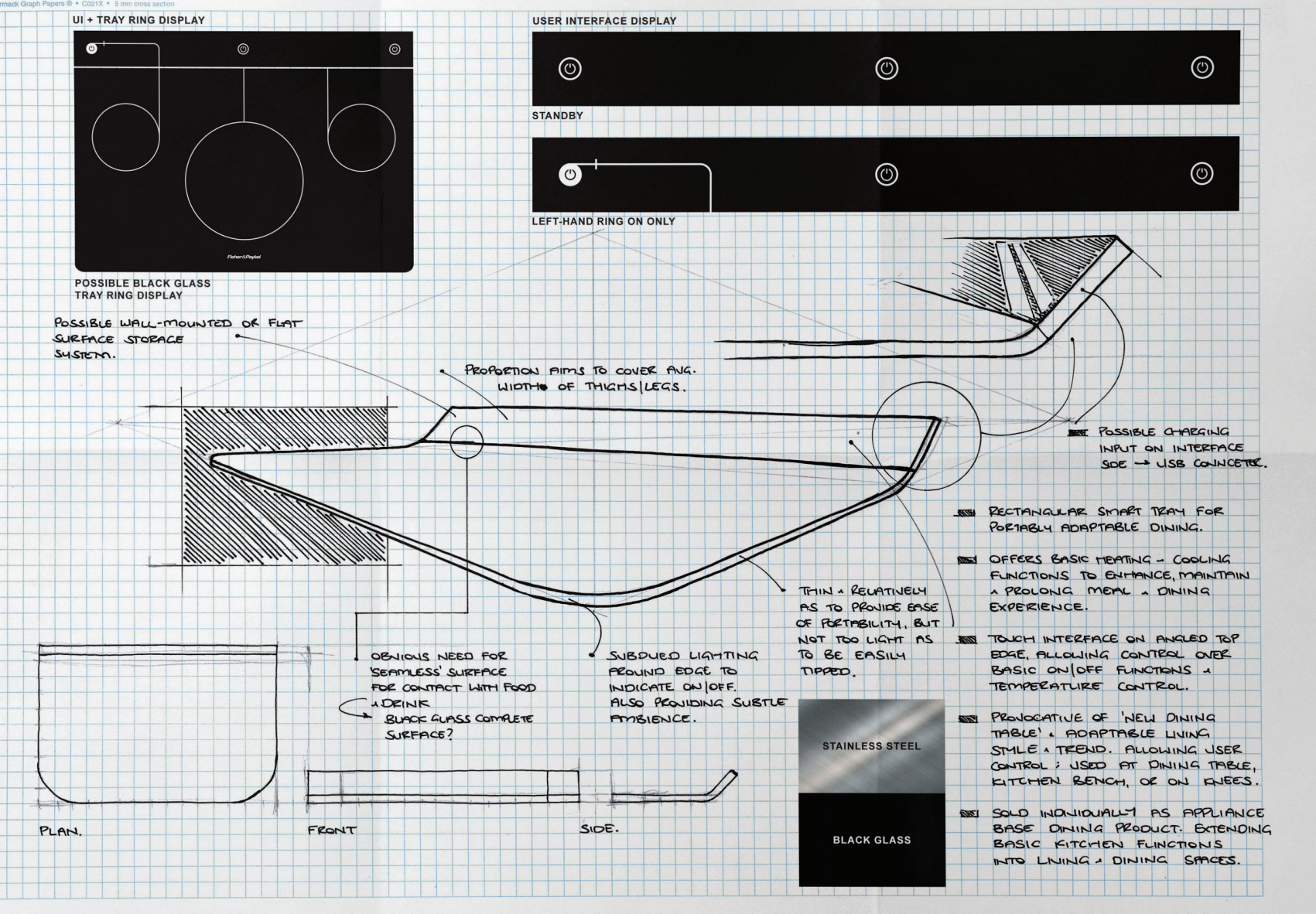

The second design experiment was an iteration on the first experiment but with particular focus on designing an appliance-based device for the possibilities of providing a device that supports basic utilities and enhances the daily patterns and behaviours of its user. The Smart Tray concep effectively sought to utilise the fundamental everyday act of dining to bridge and integrate digita technology within the scope of home appliance design. This aspect also ains to consider pre-existing as all case study households interacted with digita echnology while dining for entertinment purposes, with two of the four routinely watching the $6 \mathrm{pm}$ ews on $T V$ with dinner. While the form expresses a sleek and minimal aesthetic, its primary function centre on an induction appliance device identical to that developed in the initial design experiment. The with , more procaive finction proves the user design, essentially the convergence of an applince inspired dining tray and pervasive technolog/ nvisions the user connecting to online applications and devices in close proximity wirelessly through a seamless user interface touch screen systern.

In addition to the Smart Tray, this design de initial

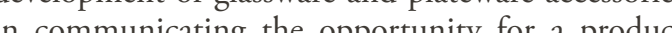
mily. The expanded conceptualisation of this speculative product sought to position the tray and associate glassware and plateware as a singular entity he user require utensils when engaging with the tray, so fi seens appropriate to supply utensil accessories

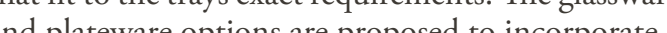
conductive matriality that takes advanto of the performance of the trays induction capabilities. In considering ergonomic portability as an essentia ement, a secondary function explores a magnetic elationship between the tray and accessory range. Whether permanent or via a magnetic off/on base the would improve the portability of the tray and

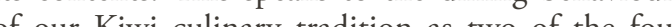
household case sudies enjoyed their diner while sting or kneeling at a coffee table.

The applicability of these functions relates to literature research on the smart home and the argument for the ubiquitous integration of
digital technology within the home through such 
conceptual thinking. As a concept driven by future
forecasting and a speculative design approach, this forecasting and a speculative design approach, this
concept seeks to propose and provoke questions regarding how digital technology and appliance design elements are integrated and utilised in the home of the future.

The Smart Tray developed within the second experiment provided a strong basis for further development, not only for the simplicity of form
but inclusion of a touch interface system enabling the seamless application of technology. At this stage it was determined that the further development of glassware and plateware should be avoided for the sake of focussing on the user interface of the Smart
Tray and in presenting a more concise and refined Thay and in presenting a more concese and refined

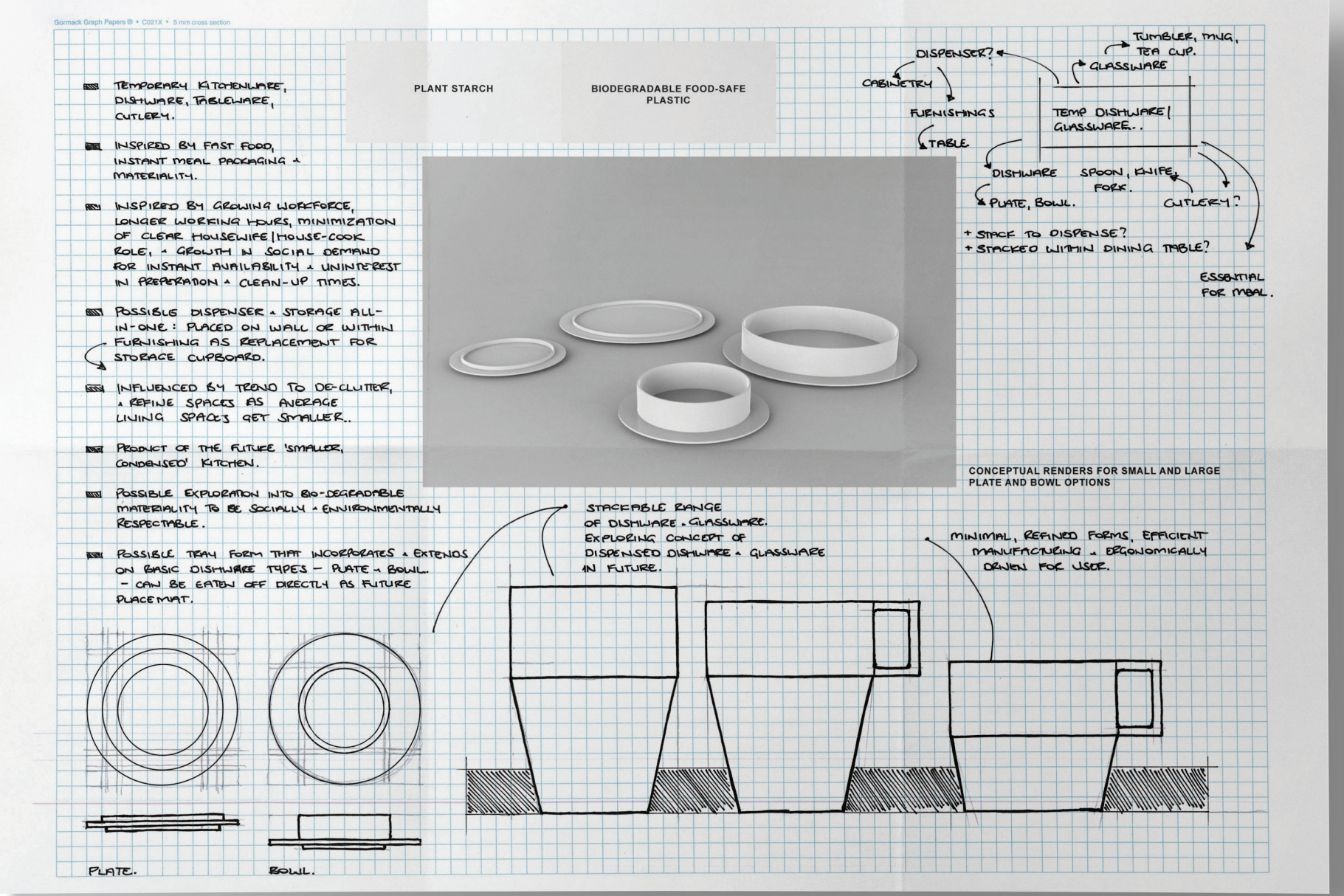



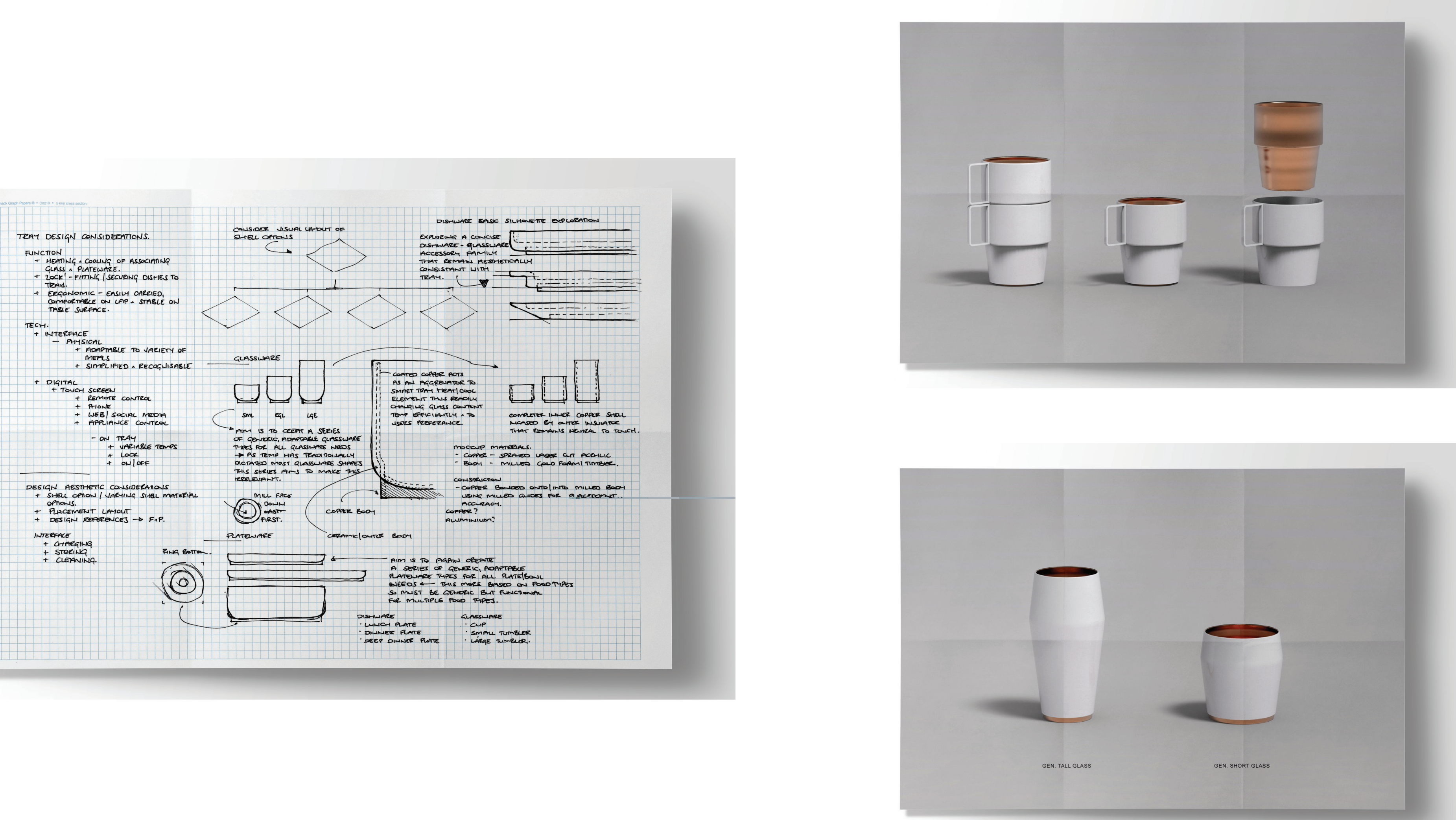
TOP: Figure 53. Generic Cup Glassware concept; sketch 005.
BOTTOM: Figure 54. Generic Tumbler Glassware conceppt 
Experiment Three

SMART TRAY ITERATIONS

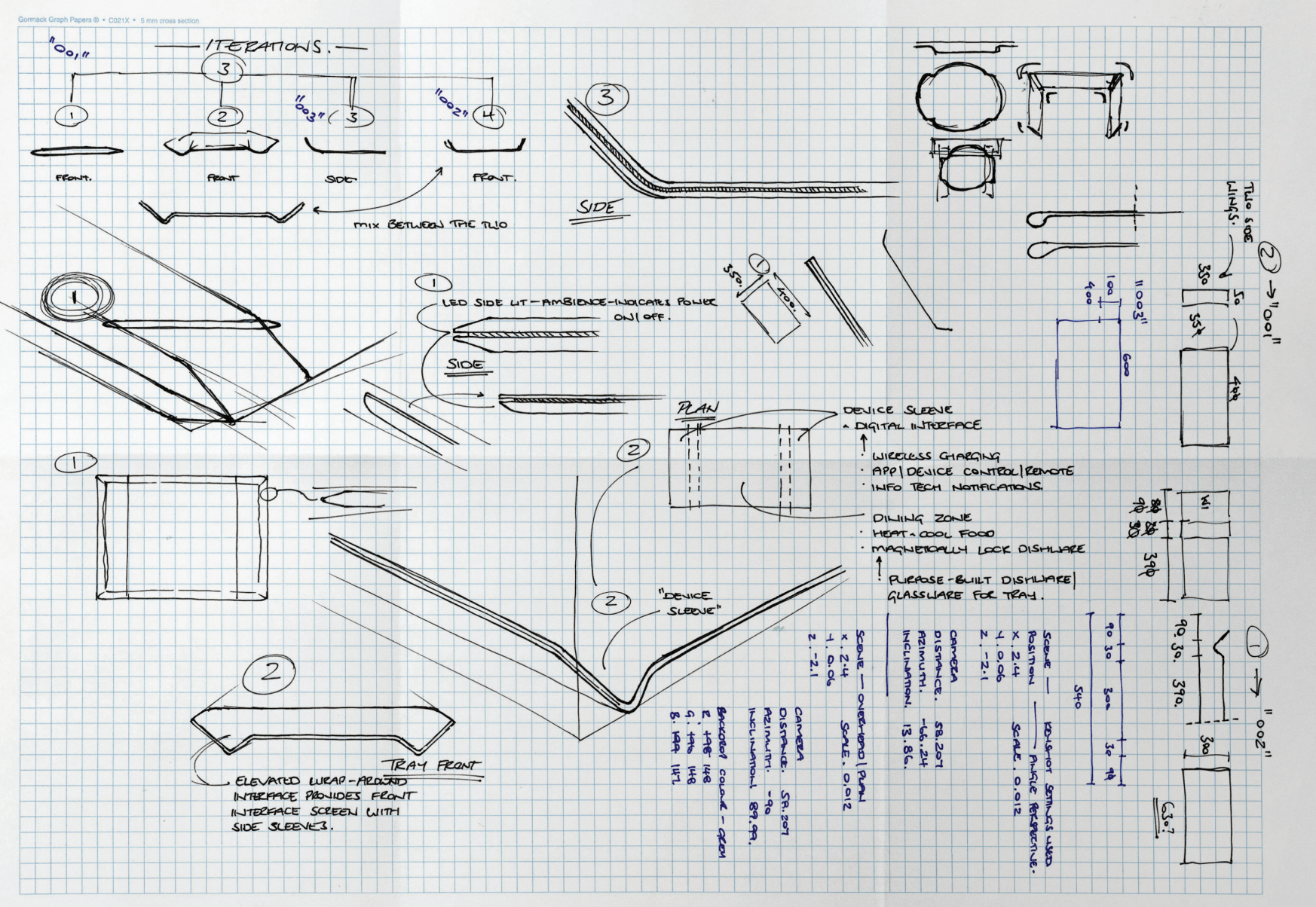

Culminating the previous design experiments, this experiment aimed to further apply the findings three iterative design solutions. Utilising coping sketching, sketch modelling, and experience prototyping methods the three iterations explored various approaches to the design of the form that effectively integrated digital and physical interfaces while maintaining a minimal aesthetic.

After sketching and rendering $\mathrm{CAD}$ representations, iterations 002 and 003 were selected for their 002 and 003 were then mocked up through laser-cut acrylic sheet and bent to to produce the experience prototypes that would support the development of the next stage of the iterative process. These basic prototypes quickly exposed the failures and successes in scale to fit a dinner phatewions picce 002 all in scalc to fi We positive ergonomic qualities. Iteration 03 was more used on a surface or lap due to the digital interface paddles on both left and right-hand sides of the tray. The Smart Tray iterations ultimately resulted in 002 being selected as the final concept in which iterative 


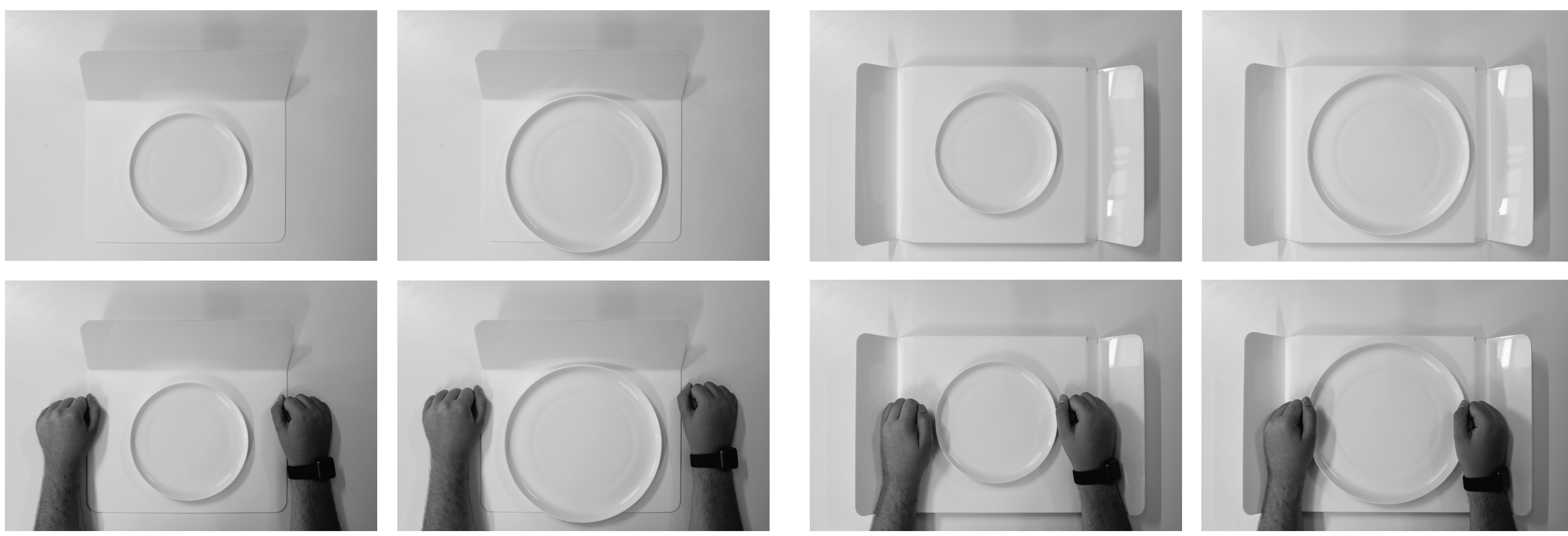
Experiment Four

DINING ZONE

Experiment four, Dining Zone, explored the possibilities for framing the most ergonomic scale and proportion for the continued development of the 003 were too small to fit the appropriate utensils the experiment primarily centred on using a plateware piece centrally within the dining zone, while having room for a glassware piece on either left or righthand side of the plateware in acknowledging both left and right-handed users. Using masking tape a dining zone was measured and recorded through photography. This experience prototyping exercise glisssware utensils in determining the minimum space required for the most effective ergonomic interaction.

Through this exercise the dimensions of the dining zone, originally identified as $500 \times 350 \mathrm{~mm}$, was Tray dining zone. This dimensior wa final Smart in Experiment Five, which will be concluded on in its own subsequent section to follow. 

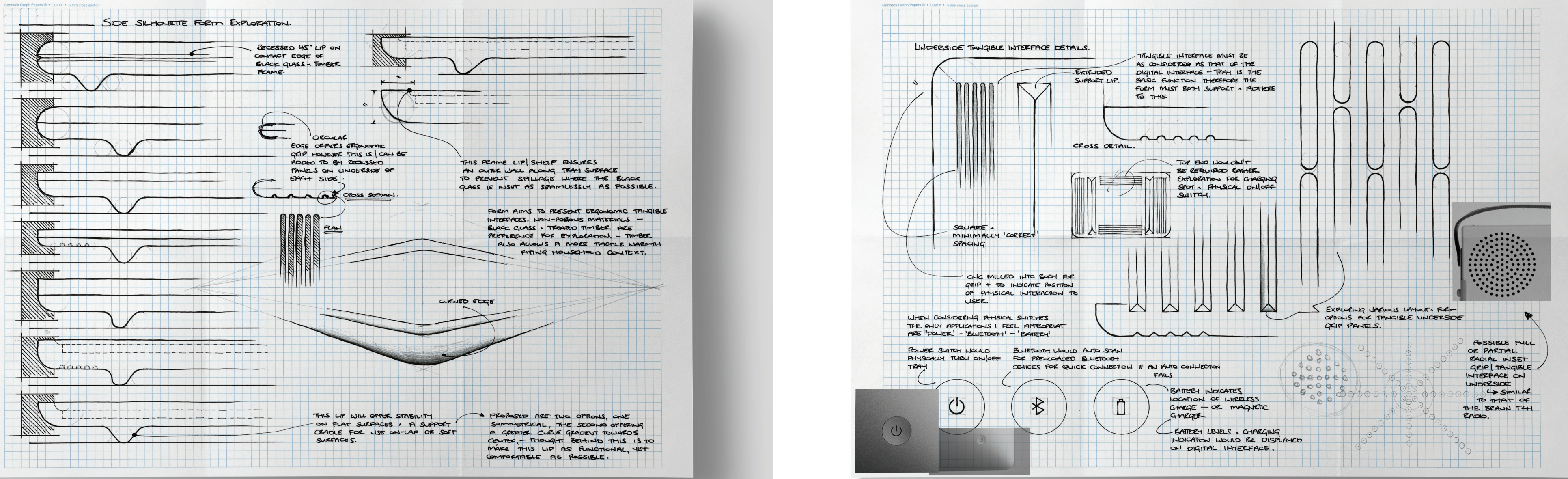


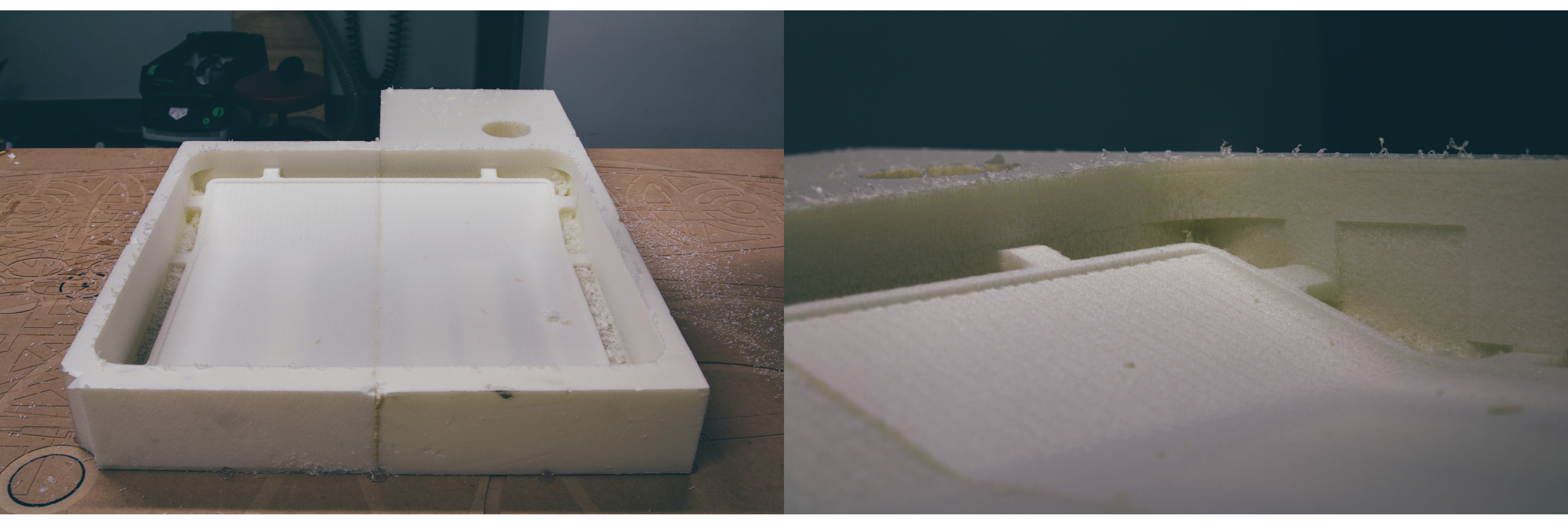




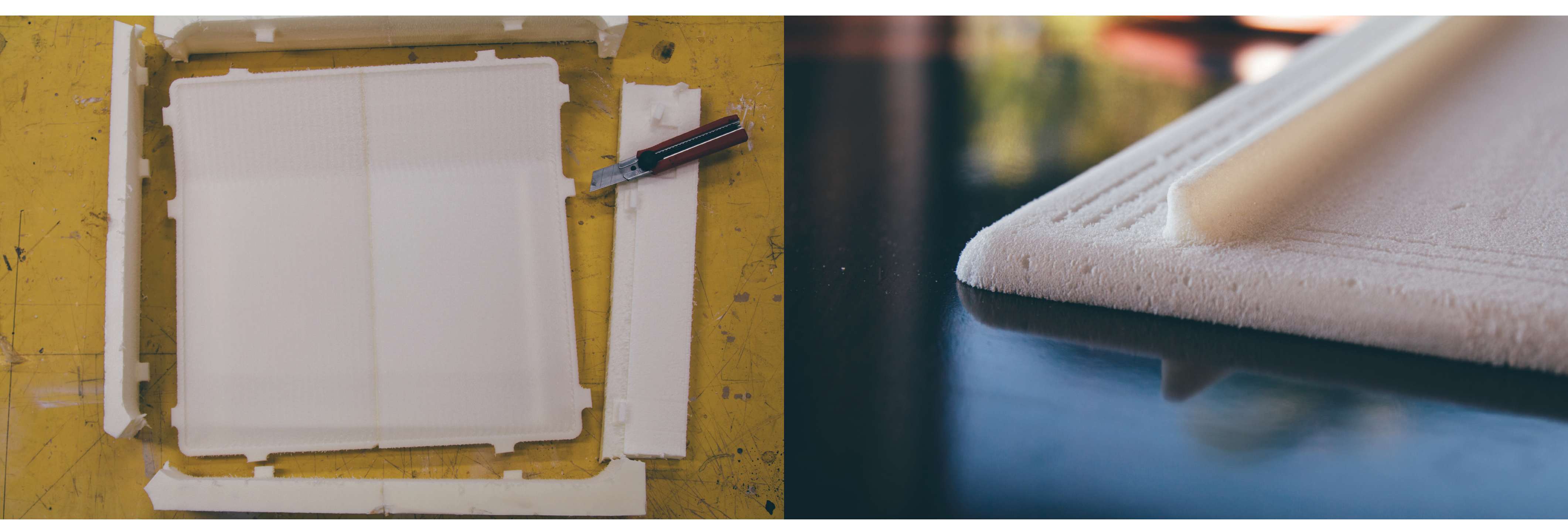




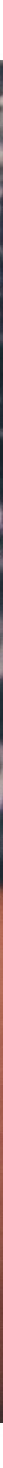




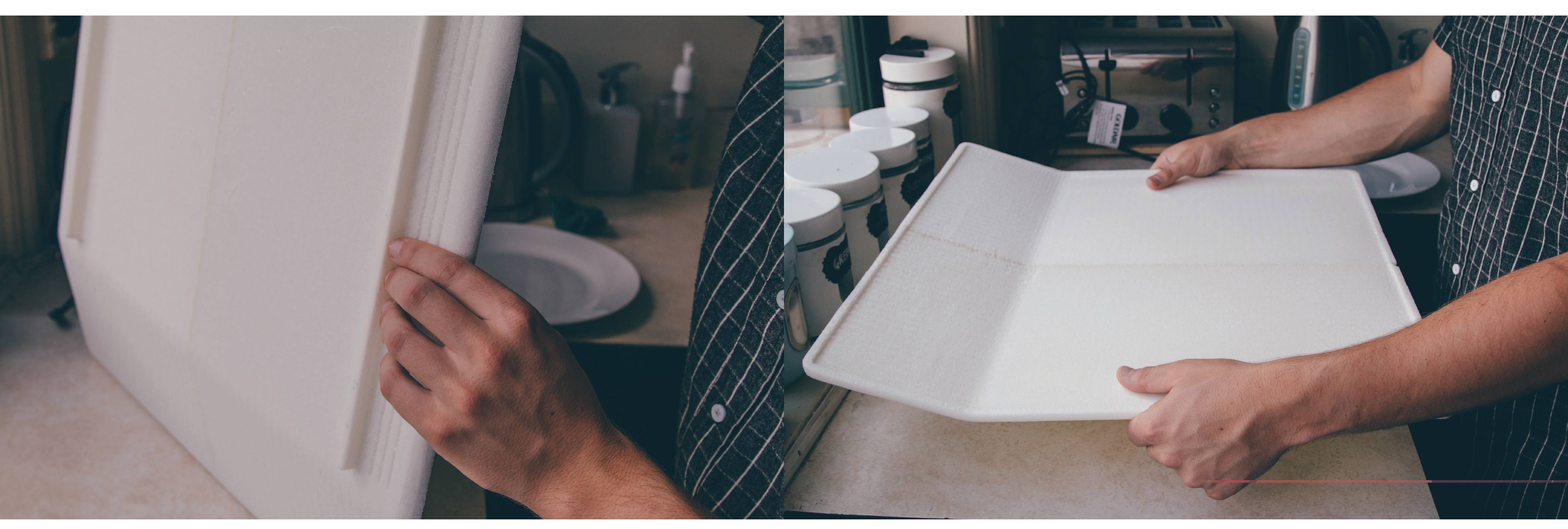


Experiment Six

\section{DIGITAL USER INTERFACE}

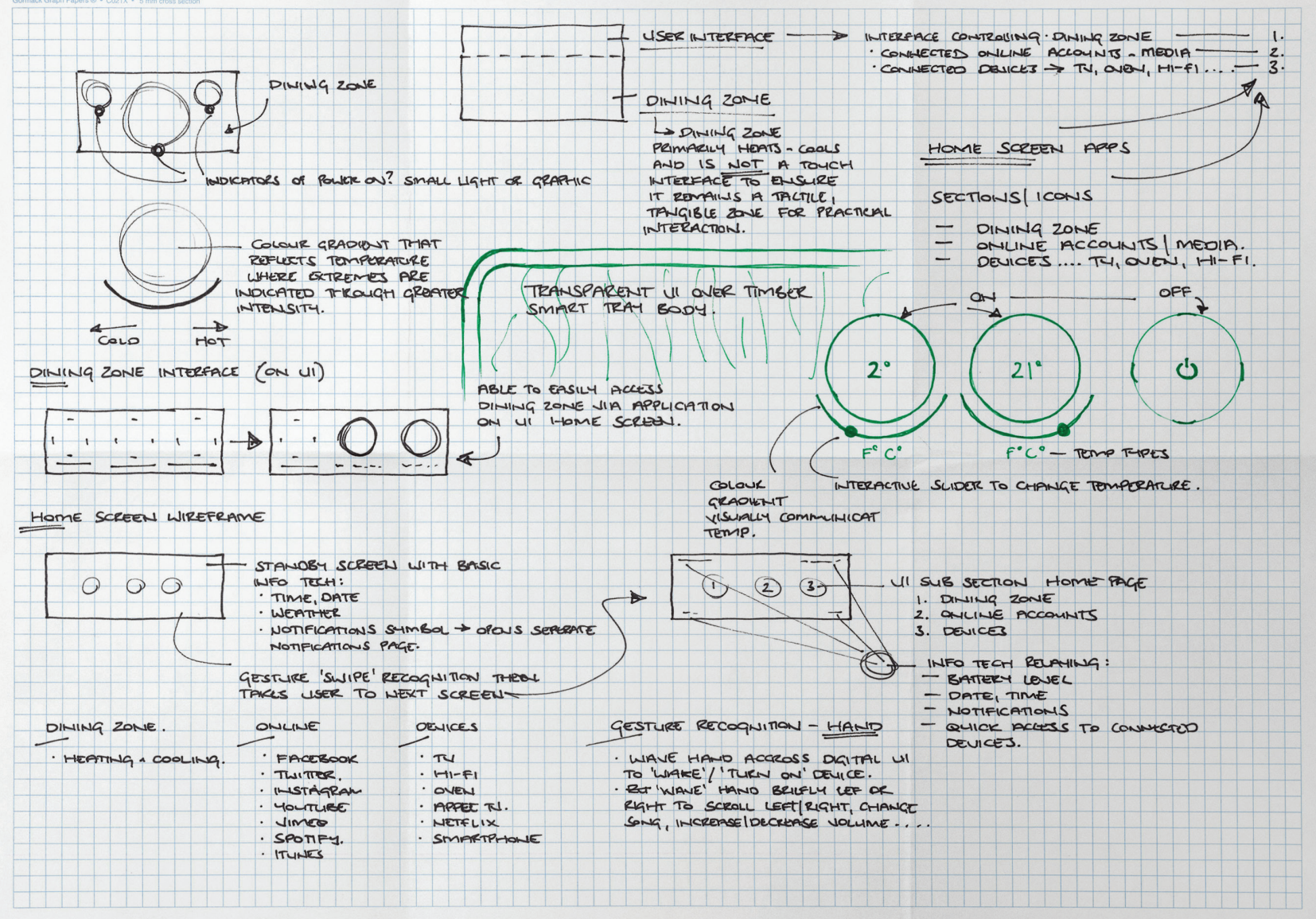

Experiment Six, although brief, began early in the conceptual stage before finalising the overall design experiment utilised the cow conceptualising the Smart Tray's digital interfod in was identified early in the research through literature and the case study series that digital technology was a critical element in producing designed solutions for the future dining experience. Therefore this experiment aimed to speculate the aesthetic qualities and basic fing of a digtal interace whin

Reflecting the research proposition in support of the ubiquitous application of digital technolog within the future domestic dining experience, the conceptualisation explored seamless, minimal

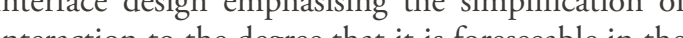
not so disant futue The primary method anged during this phase was concept shething throgh which eesthetic tresments and wireframes were envisioned. The development of the interface design was directed to a large extent by literature and case study research, particularly in breaking down the various interface functions into three core zones: (se fist of these, he thing capability in allowing the user full temperature control of the trays dining zone surface through the cor their personl online accounts such as Facebook to Instagram, as well as online entertainment and news. The device zone, the last of these zones, allows a user wireless control of Bluetooth connected devices in the tray's proximity - from the oven in the kitchen, to the TV in the lining space. The hiree core sections

Experiment Six embodied an iterative design process resulting in the final iterations implementation within the final Smart Tray design solution. In devising a strategy to refine and present a speculative experience prototyping would not take place at his stage for two reasonst to begin withe de solution while prototyped to full-scale, was unable to show a functional digital interface. Additionally the digital interface aimed to provoke future possibiliti for the application of digital technologies within the home, however user feedback on this point was not of substantial value to the development of the final ign in relation to the research intentions. 

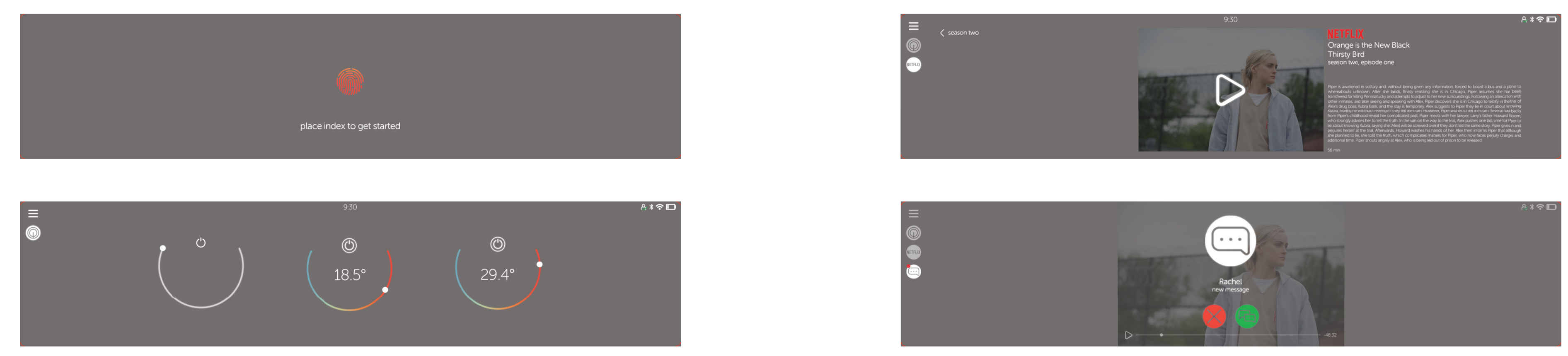
DESIGN SOLUTION

The Smart Tray represents the final design solution of this research in considering the impacts of technology on the New Zealand domestic dining experience. Seeking to meet the future needs and
desires of Kiwi households the Smart Tray provides a portably adaptive appliance device utilising the a portably adaptive appliance device utilising the
fundamental human act of dining to speculatively fundamental human act of dining to speculatively
bridge innovative kitchen appliance design, and digital technology design thinking as a means to advocate for greater ubiquitous application of pervasive technology within the future home. 


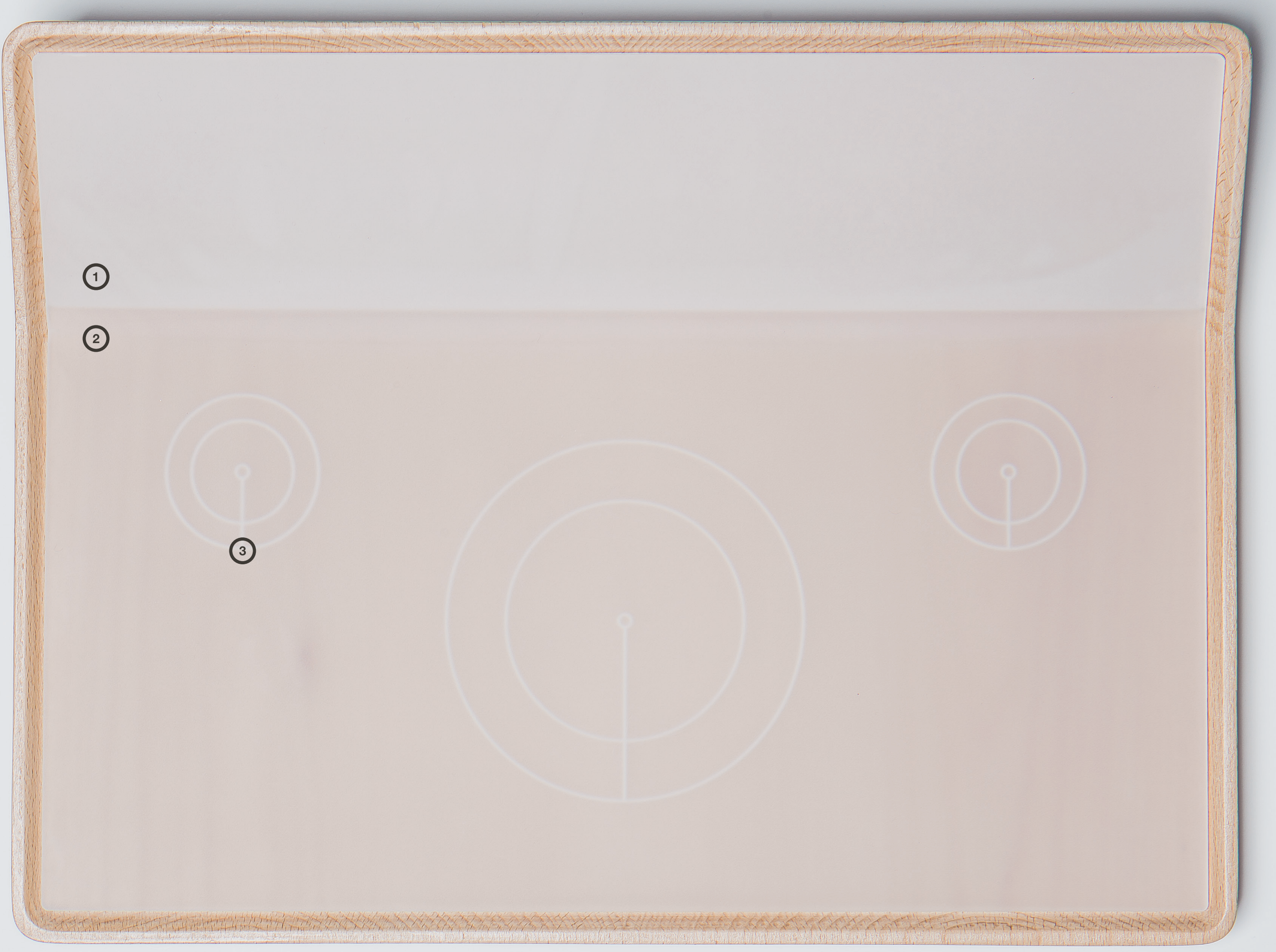

1: Digital Interface Display 
The Smart Tray presented in this thesis represents the final design solution of this research process. In seeking to meet the needs and desires of Kiw
households in the future, the design solution proposed here in the form of the Smart Tray provides a portable and adaptive appliance-device increasingly technology-reliant lifestyles. The Smat Tray design satisfies a multitude of needs associated to the fundamental human act of contemporary dining in regards to digital connectivity, highly considered interaction design, and the application of pervasive technology. This design output speculates on ideas that capitalise on and perpetuate the use of hat have the potential to propel innovative kitchen appliance design today.

The literature reviews, case study findings, and user esting and feedback have guided the developmen of the Smart Tray and have provided substantia marketable product In tying this final design back to the literature reviews conducted as part of this research, these elements acknowledge the kiwi culinary tradition and evolution of Kiwi domestic kitchen and living spaces from the early twentieth century. This design reflects a flexible dining opportunity affording the user complete control in front of TV or at a dining table.

The final Smart Tray expresses a minimal, elegan form that seamlessly integrates physical and digital interface elements. The physical interface encompasses the tray's side edges and underside elements, where filleted side edge details produce comfortable in-hand tactility for the user. The his tactility providing the usor an edged surface with which to grasp the tray In developing the trays scale considerations have again been drawn from the research findings with particular emphasis on the evaluation of the extruded polystyrene Smar Tray prototype in Experiment Five. Feedback from this experiment centred on the tray's necessity to communicate a thin, light, rectangular proportio for the organisation of a traditional dining-ware hyout: the dining zone is therefore defined by the space allocated to a central plateware position with wo glassware positions slightly further from the use on both left and right-hand sides of the plateware,

making the tray proportionally ergonomic for daptive use, from its position on a user's lap to placent on a dining table. Extruding $12 \mathrm{~mm}$ 'feet' act to tise sure of the tray, two elongated fat surface in order to communicate and frilina the tray's portability. The 'feet' also act as support guides when placed on a users lap, whereby the filleted surface edges allow a user's lap to nest with the bottom form of the tray. The far edge of the Smart Tray angles upwards by twenty degrees in order to provide the user a more ergonomic view of the digital hiterface display. This angled display he dining zone and digital interfece dishy

In breaking down the compositional elements making up the Smart Tray, the materiality ascribed to the final design has been critical in emphasising the human-centred design attributes of this project. As an appliance-device it was evident that sense of warmth and personal companipress conjunction with qualities of functionality and durability. For this reason the Smart Tray's body is constructed from Beech, a timber commonly found in international furniture and kitchen utensi markets, as it is valued for its durability. This choice of material also acknowledges New Zealands strong forestry representing one of the earliest ind wit in the nation's forming The ability to merge echnology with a traditional material such as wood was chosen intentionally as a way to integrate pervasive technologies within the future home while parting a connection to the familiar attributes

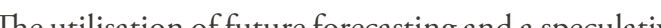
design approach afforded the opportunity to push boundaries and suggest provocative treatments of the interactive display and components and pervasive a transparent digital interface was an element incorporated in the Smart Tray's design relatively hate in the final design process. This was ultimately apsied because it was dects. This was the black appeared to emphasise a commonplace induction appliance product rather than a seamless digit interface-tray relationship. The transparent digita display also supports the intention of minimising he visual impact of digital interfaces through a components.

Asstated in the section that explains the developments explored in Experiment Six, Digital User Interface. the speculative functionality of the digital interface online zone, and device zone. The dining zone represents the heating and cooling areas on the tray's surface incorporating a centrally positioned area for single plateware, and two smaller glassware area positioned slightly further from the user at both eft and right-hand sides. This refined layout reflects contemporary Kiwi culinary traditions represented by on this was observed in the Silent Generation household who admitted to sporadic use of a side dish associated with more traditional British mea structuring. The online connectivity zone utilises wireless Bluetooth connection to the user's smat phone, in turn connecting the Smart Tray to their online accounts. As smart phones will hevitably Tray's online connectivity foresees acting as a digital utility device to enrich a user's dining experience. The device connectivity supported by the device zone will build on to the functionality offered by wireless Bluetooth connectivity as pervasive technolog increasingly takes the form of tangible products an appliances. Pervasive technology is already found in

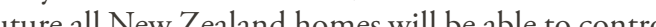
kitchen appliances such as the oven and fridgereezer using this technology, as well as living space products that include the TV and hi-fis systems. The Smart Tray's wireless connection to a $T V$ as part of the device zone, for example, would also allow the wireless connection to an online streaming platforn the three zon that mate up the digial intef on the amalgamated reasoning that has shaped the interface aesthetic.

The Smart Tray's digital interface communicates its abiquitous application through a minimal desig predominantly monochromatic colour scheme and ayout in order to express elements of empathy and docility towards the user. Through this quiet aesthetic experience the Smart Tray seeks to incorporate pervasive technology within the dining experience, rather than vice versa. The interactional qualities of the Smant Tay have also been explored where been incorporated into the final design solution Gesture recognition can be considered a component of a perceptual user interface (PUI), effectively allowing a computing device to mathematically interpret human motion (Rouse, 2015). The Smat Tray proposes simple user gestures such as a full hand swipe across the digital interface display in pplication of perceptuat user interface tech acts to enhance the tray's interactional qualities hrough non-touch navigation of the digital interface display. Alongside more common touchscreen capabilities, gesture recognition provides the use with an ergonomic digital interface display.

Photography has also been an important elemen in presenting the Smart Tray's physical and digit human-centred design solution. The contextual photography follows a model user throughout typical day according to their dining experience. Presented in an overhead view to humanise the tray's interactive qualities, breakfast, lunch, and dinner contexts are explored through various physical and convey the tray visually. 


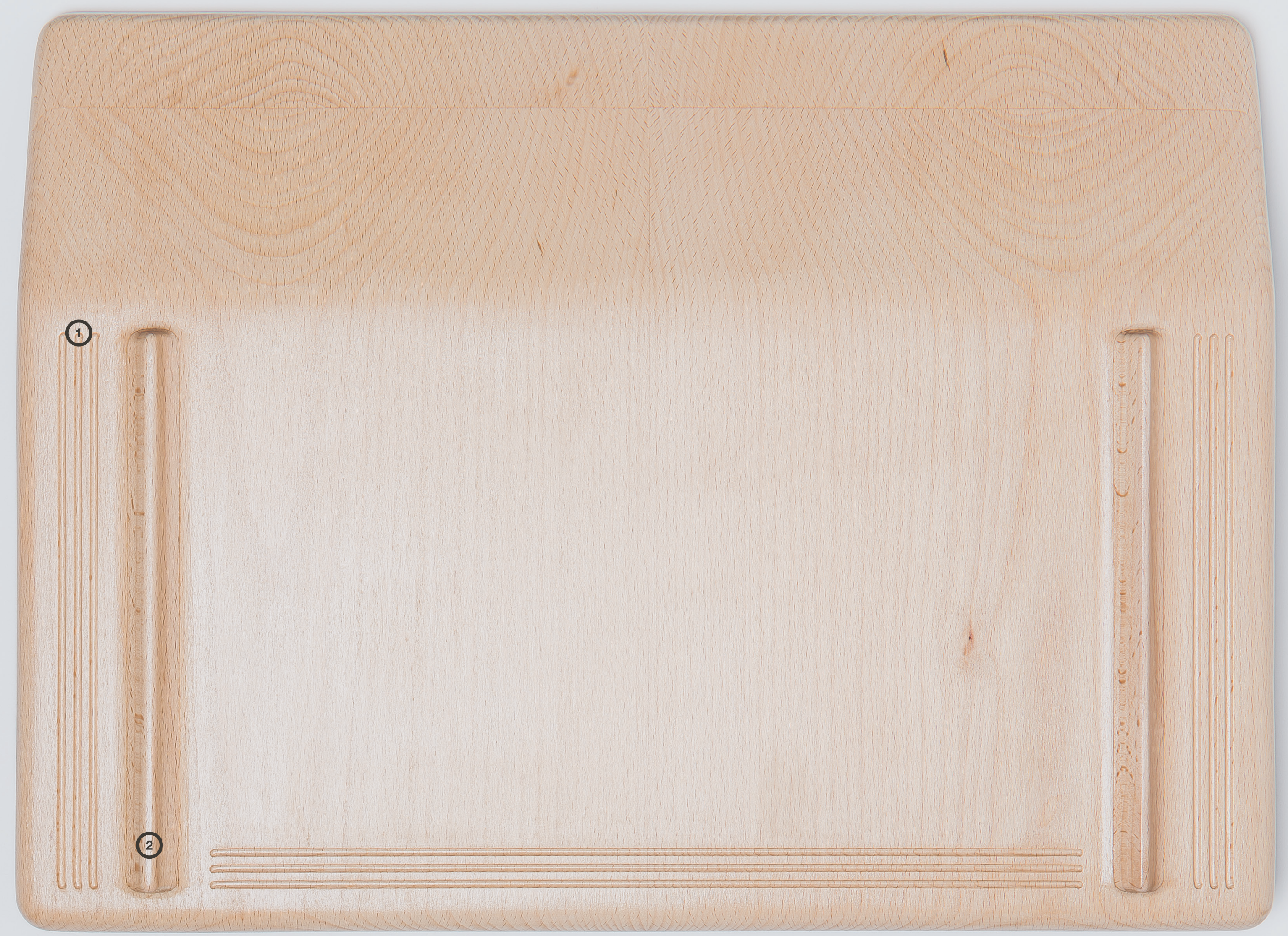


Once the user has unlocked the device they have decided to touch the list button, part of the tray's
touch screen digital display. When activated this

button in displays all applications set horizontally in

sub sections from botton:

and device zone. Alternatively when touched again

return to the previous standby screen.

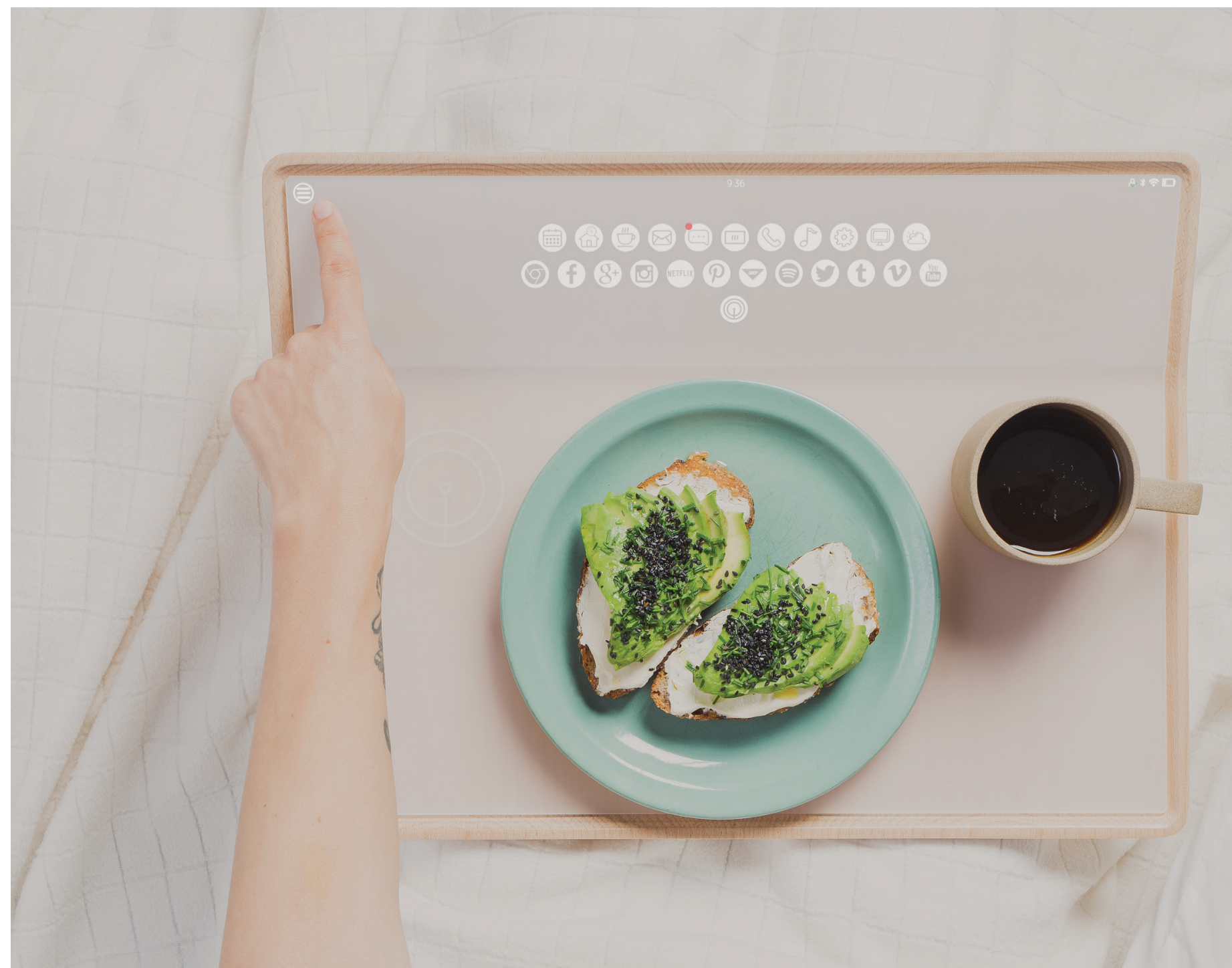




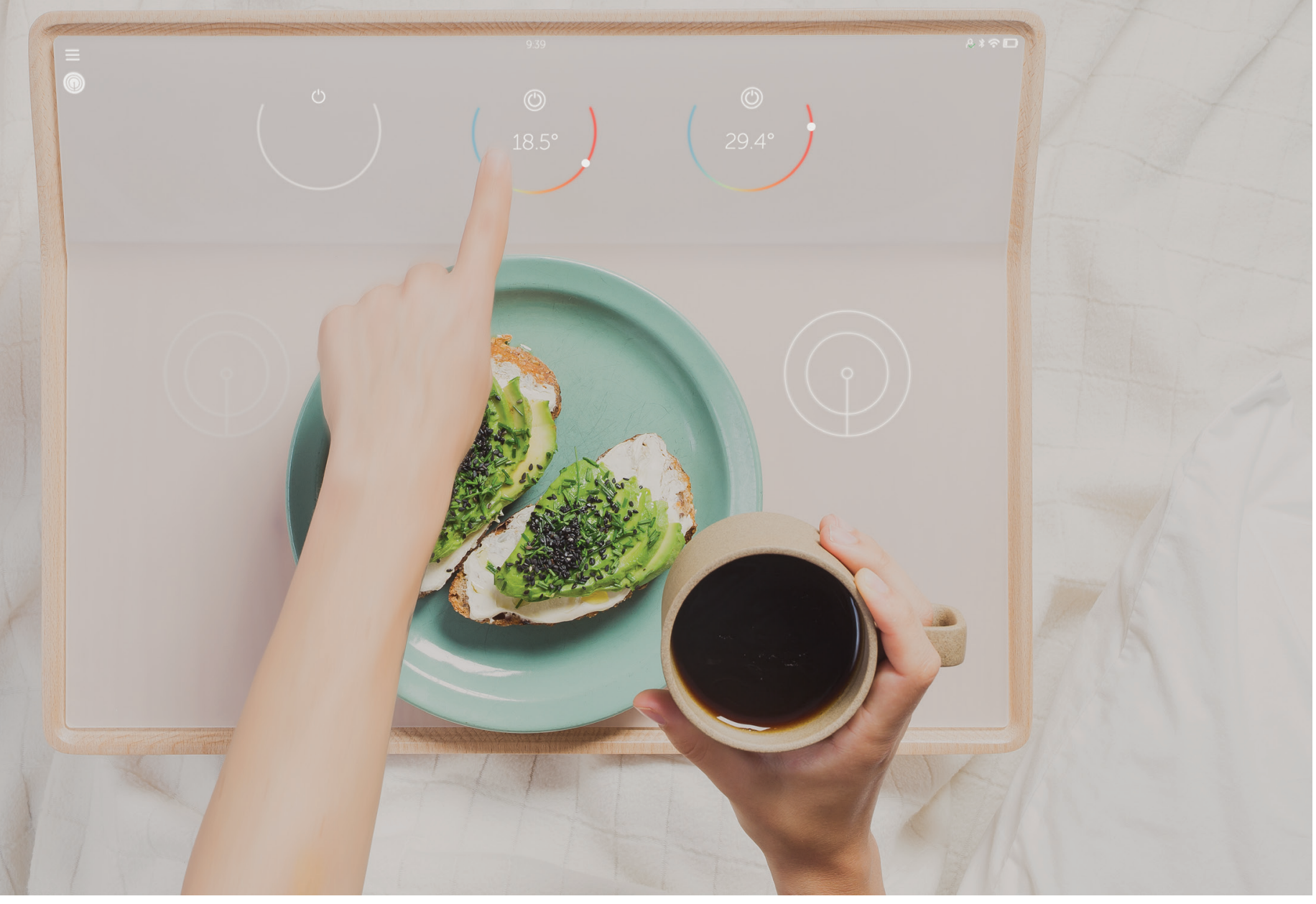

Using the tray to eat breakfast in bed, the user has now opened the dining zone application, turning
both centre and right dining zone plates on. The

user has set the plates to the desired temperature, heating both their avocado toast and filtered coffee, In addition to the digital display graphics indicating a plining zone pate will ale beran to illumine physical 
Now that the dining zone plates are keeping the user's breakfast warm, they have decided to watch and selected the Netflix application, bringing up a TV

show of interest. The applications activation is evident

via the sidebar situated directly below the list button

whe the ding zone applicaly bean be seen slighty

dimmed to communicate its background use.

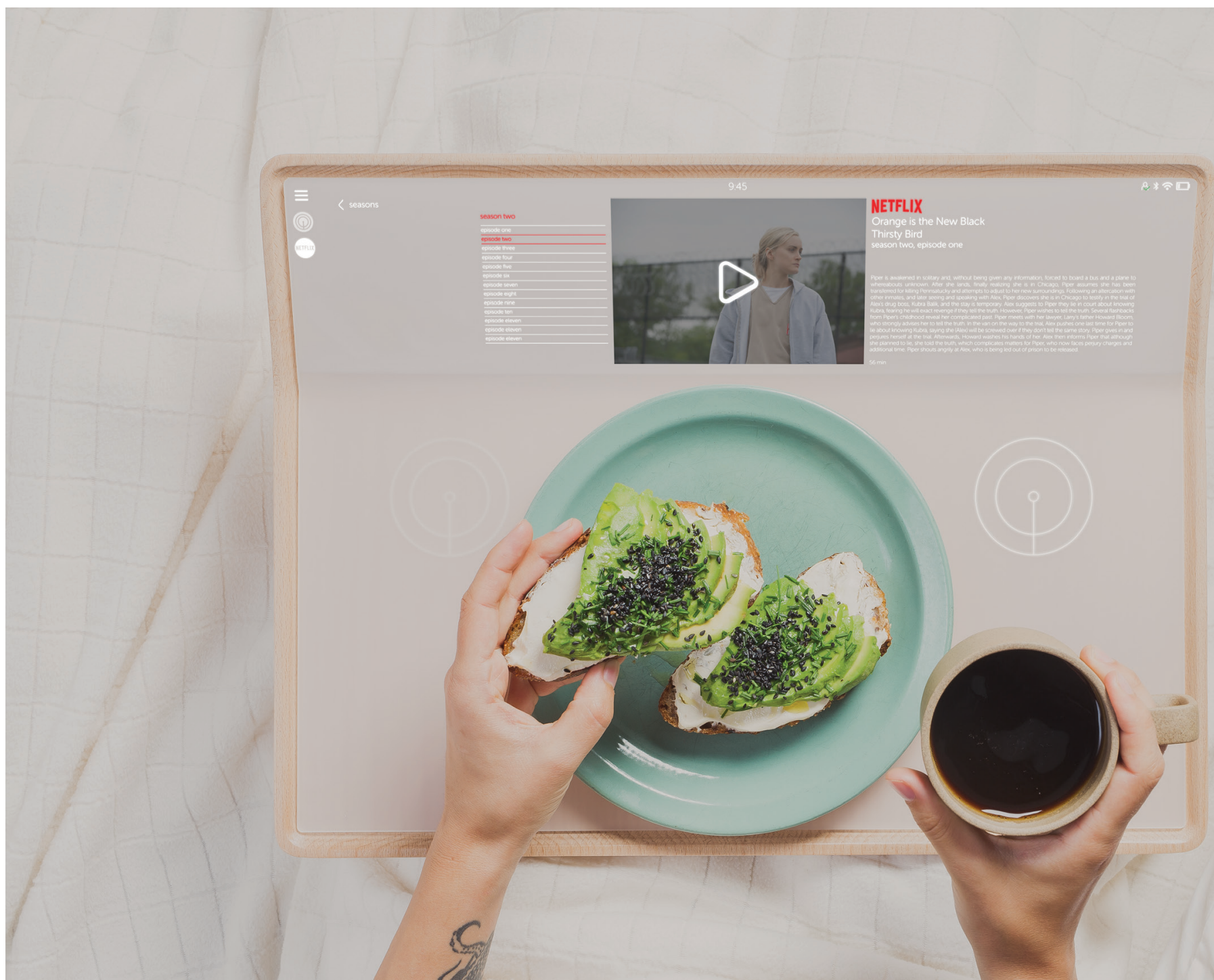




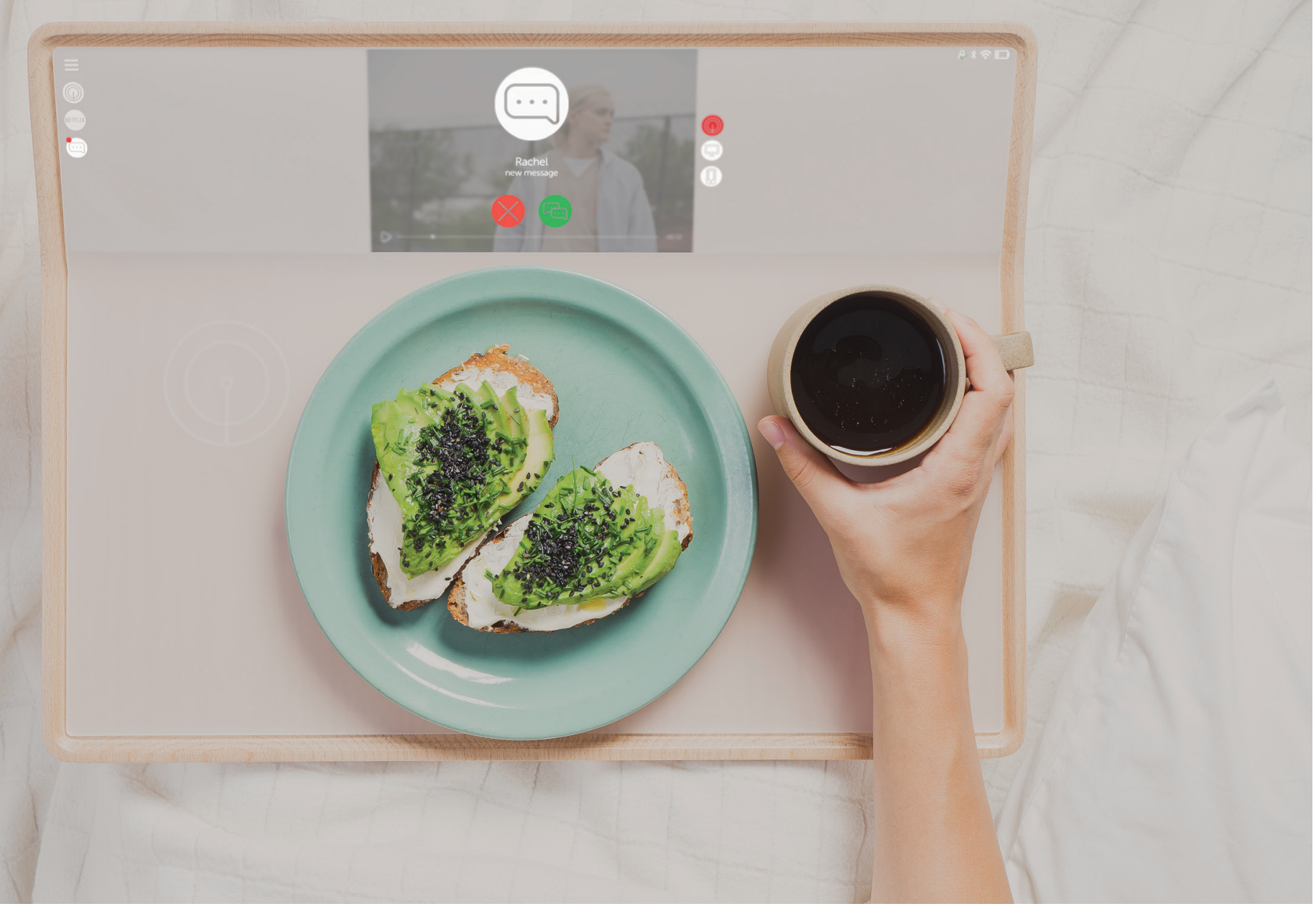

Because the user's smartphone is connected to the tray, a new message from a friend is promptly
displayed on the tray's digital display, and overlaid

over any other active application in allowing the user

instant online connectivity. The message application

can be seen in the sidebar with a small notification

cignal, which wh conthe to be dsplay not 4

message is read. 
For lunch the user has prepared some mussels and a
glass of beer. Eating from the tray the user has again

opened the dining zone applicallon and begun to se

the applicable dining zone plates' temperature. In this

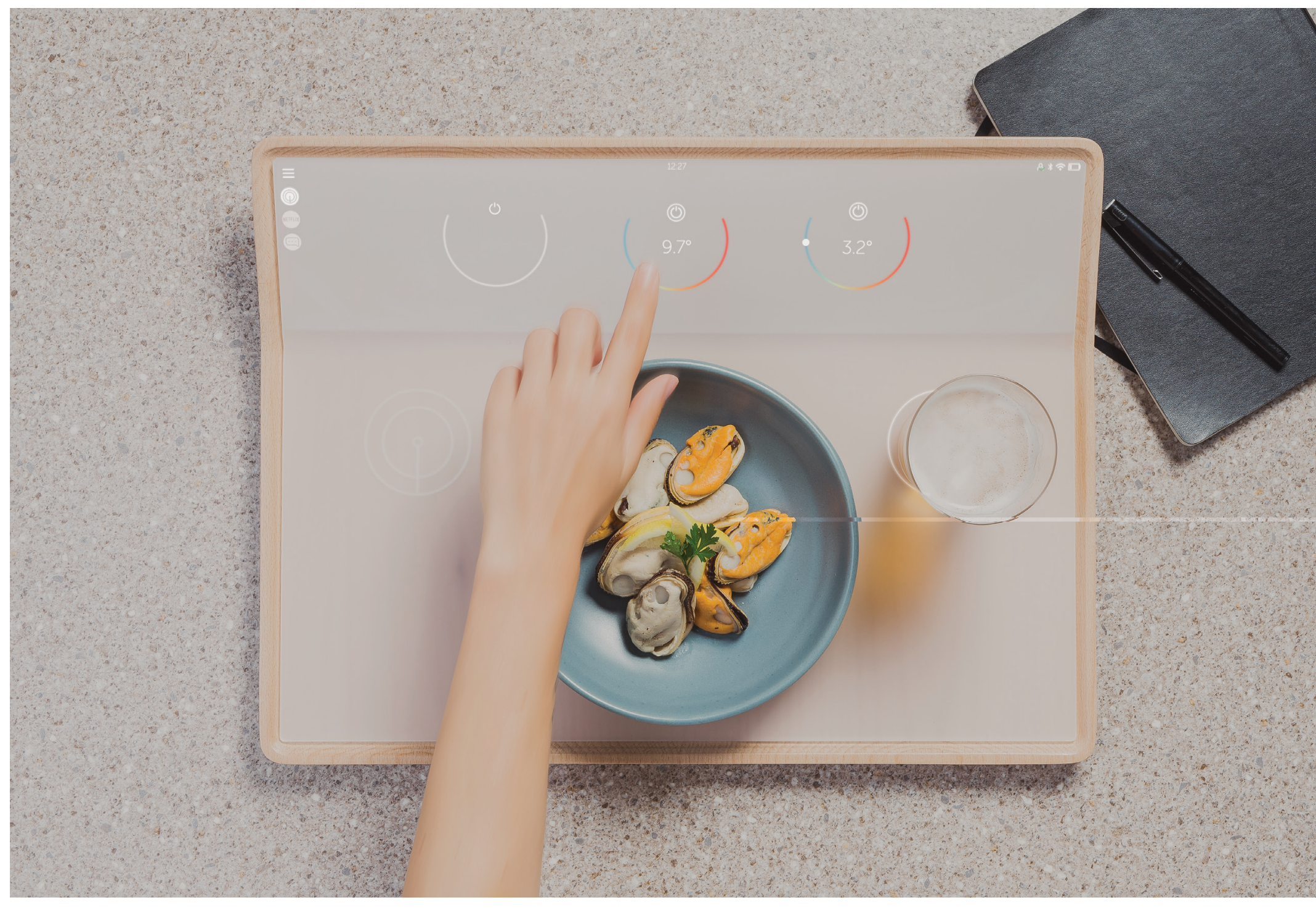




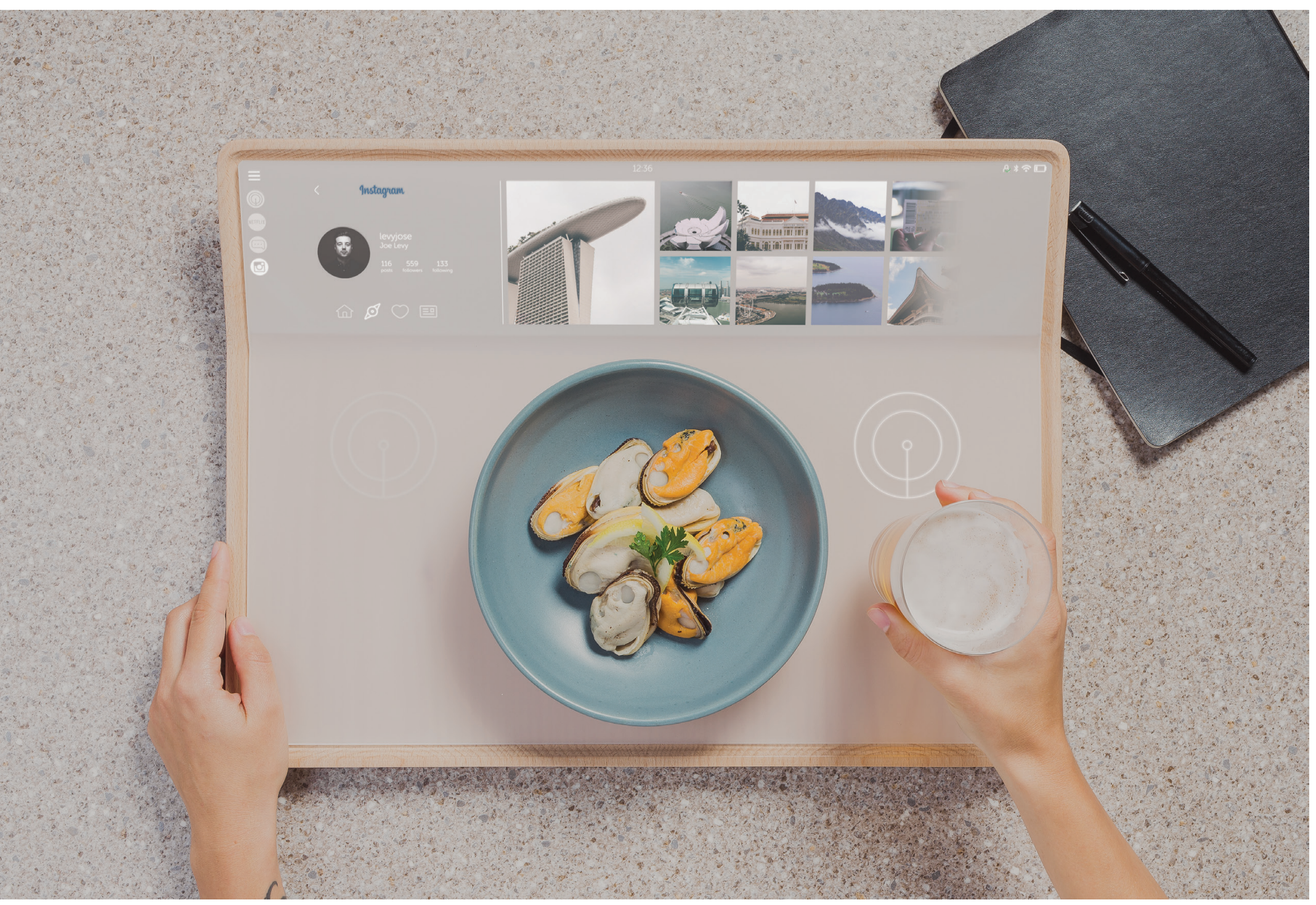

Now that the user can enjoy their meal at a regulated temperature, they have opened Instagram as part of The sidebar indicates that applications from breakfast earlier that morning are still open and can also be instantly opened if needed, where pressing on any to close it, removing it from the sidebar. 
While eating their lunch the user has opted to

play some music, connecting to the music device

application and selecting to link it to an online music

user has also selected their hi-fice Spolity. The

selection of available devices connected to the tray and the user's smartphone.

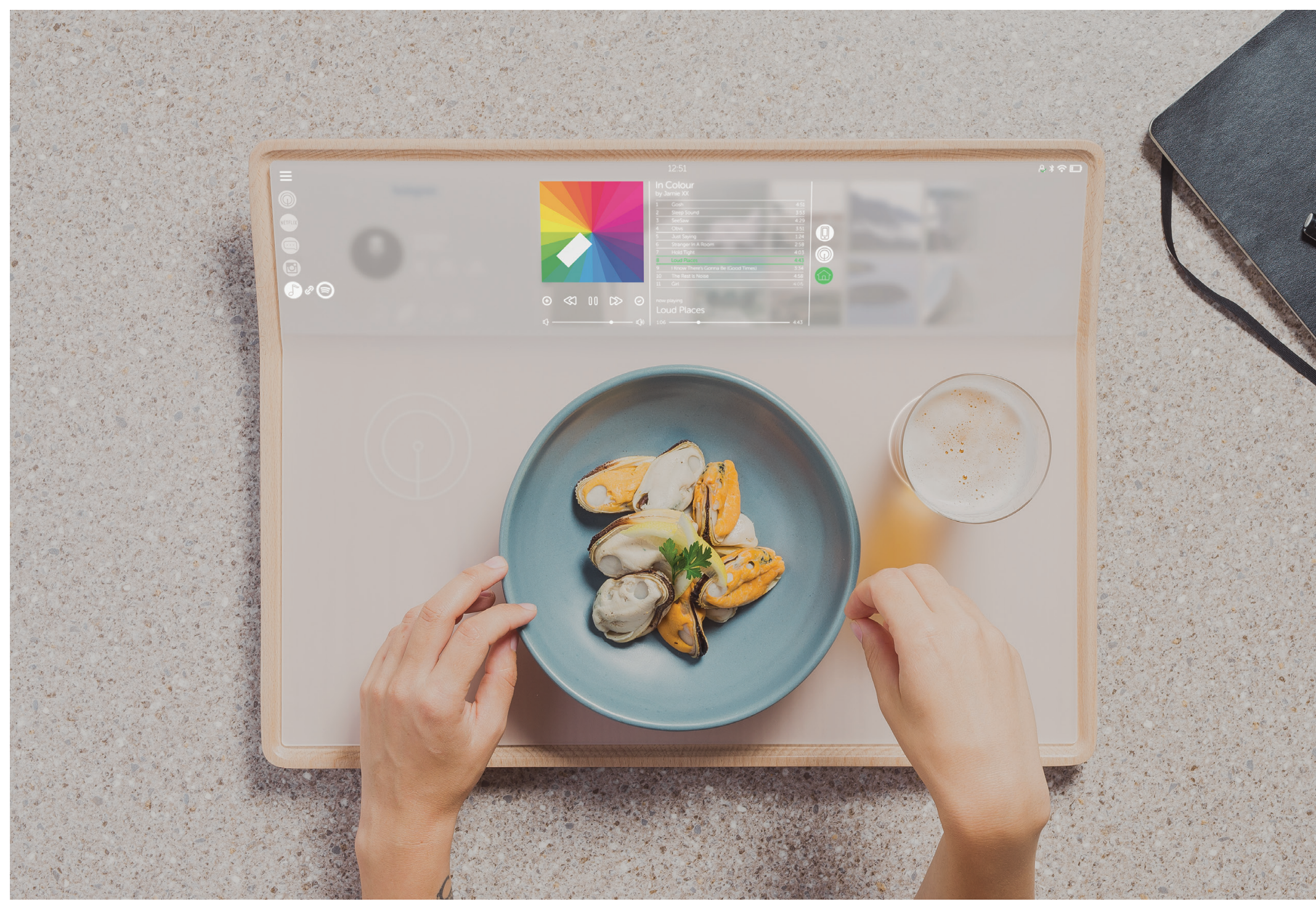


The user has served their dinner around the viewing

of a rugby game on $\mathrm{TV}$, where they have opened the

TV device application. Linking it to their SKY online
application the user can select the applicable rugby

game and watch it on their connected TV all from the

Smart Tray.

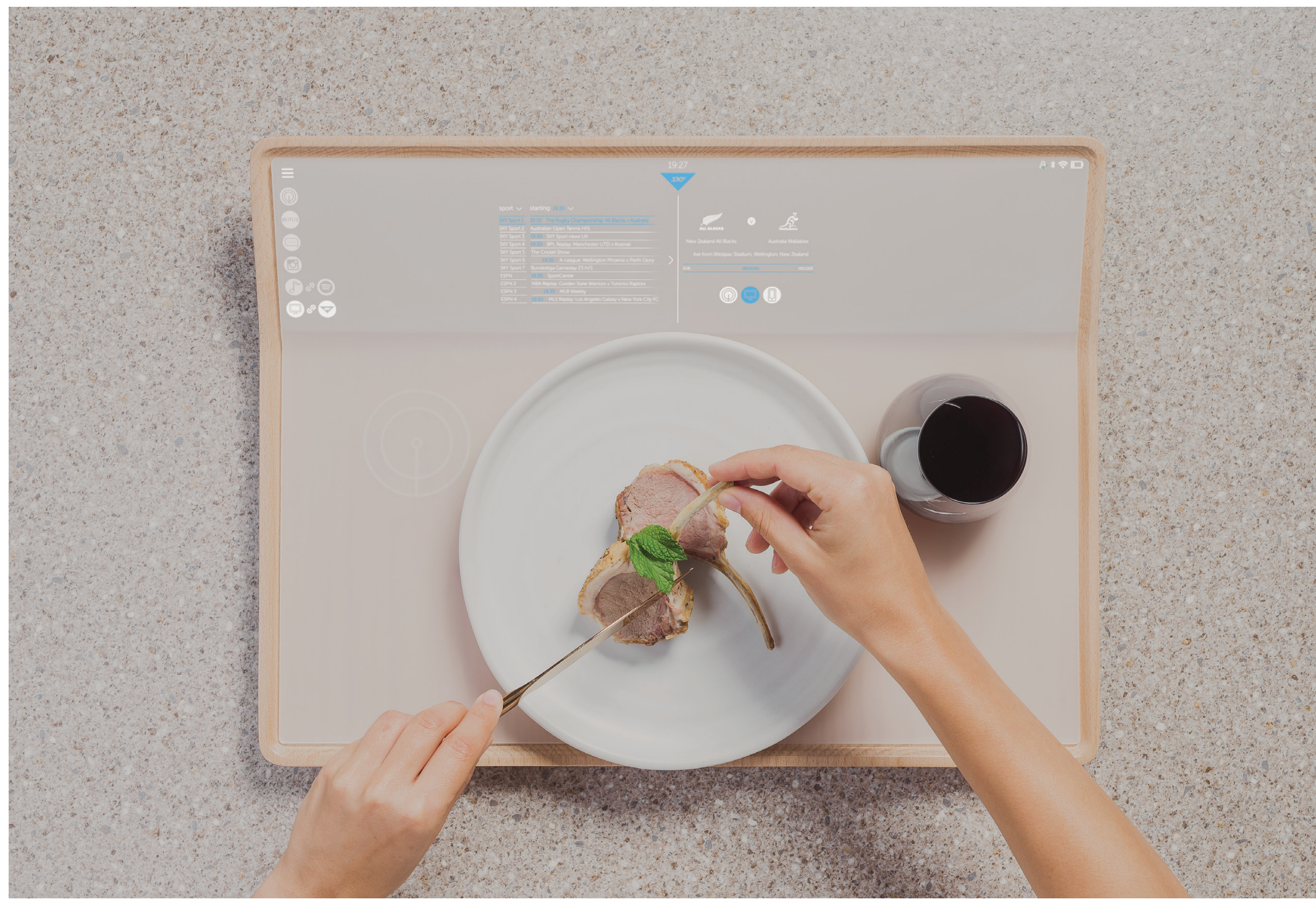




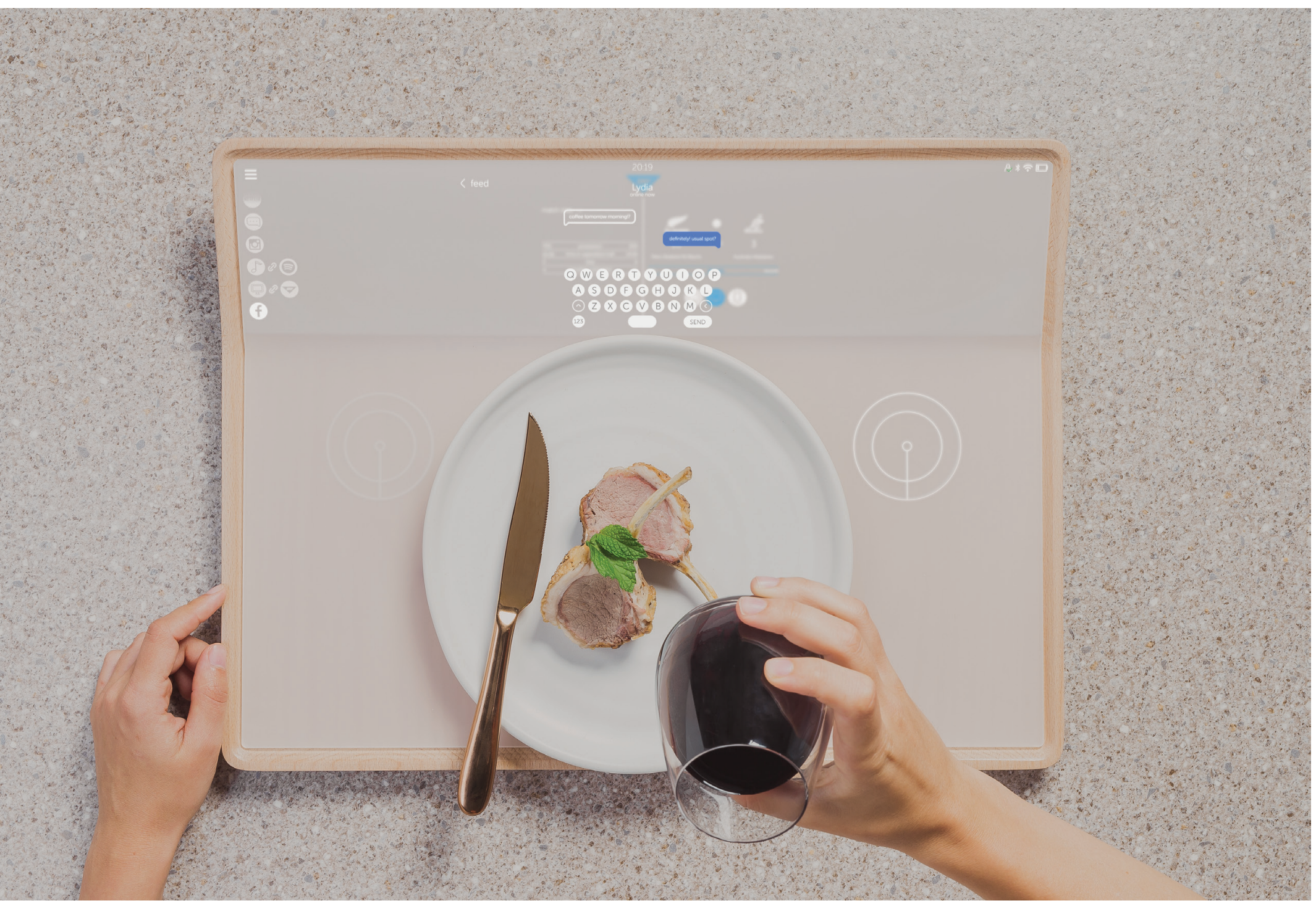
During dinner with the Smart Tray the user has received
a Facebook Messenger chat from a friend. Through the connection to the user's smartphone and online to open the application and reply organising a coffee the next morning. Meanwhile, the connected TV and SKY applications continue to run in the background. 

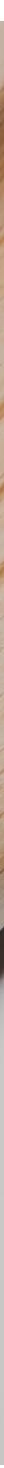
The Smart Tray is a design solution that meets the needs and desires of future Kiwi households. Although this research is contextualised within
New Zealand, the general research outcomes are New Zealand, the gencral cesearch outcomes are asplicable to a wide resule design of the final Smart Tray, is intended to offer valuable critical perspective and viable future design solution that will aid in furthering the professional field of dining design.

This research project has been completed to the highest level possible within the restraints of the the opportunity to critically reflect on the entirety of the project has allowed the opportunity to consider possible ways to extend and further explore this research topic. While the case study research has provided numerous successful elements in the Smart Tray's development, the case study research

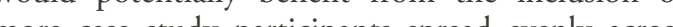
all four generations. The utilisation of a lass participant pool could have offered greater insight into contemporary elements of meal preparation as well as dining and tech etiquette behaviours, and the research finding and related design outputs.

The future forecasting and speculative approaches incorporated within this research-led design terspective and poin appliance design, dining design, and technolog design sectors. In proposing the Smart Tray as speculative design solution, the tray contributes 政 design and sociology. It is particularly surprising have such a great potential for product developmen within commercial design sectors. In the immediate sense this research hopes to engage the reader and to provoke thought about the future of design. The future forecasting and speculative design approaches utilised in proposing the Smart Tray ain

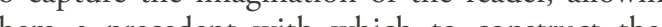
them a precedent with which to construct their Kiwi dining experience and integration of pervasive digital technologies may evolve. 



\section{CITATIONS}

Bell, G., \& Kaye, J. (2002). Designing Technology for Domestic Spaces: A Kitchen Manifesto. Gastronomica, 2(2).

Boyte, T. (2016). Mobile Keeping Kiwis Connected. Nielsen New Zealander Connect Consumers Report Retrieved January 20,20
kiwis-connected.html

Coffin, R. (2013). The role of Māori teachers in English medium primary schools. Retrieved January 8, 2016. Distracted by Technology at Mealtimes - It's Not Who You May Think. (2015, November 24). Retrieved January
20, 2016, from http:///www.tinelsen.com/nz/en/insights/news/2015/distracted-by-technology-at-mealtimes-its-

Dunne, A., \& Raby, F. (2008). Fictional Functions and Functional Fictions. Retrieved February 10, 2016, from aby.co.uk/content/bydandr/46/0

Dunne, A., \& Raby, F. (2013). Speculative everything: Design, fiction, and social dreaming. Cambridge,
Massachusetts: The MiT Press.

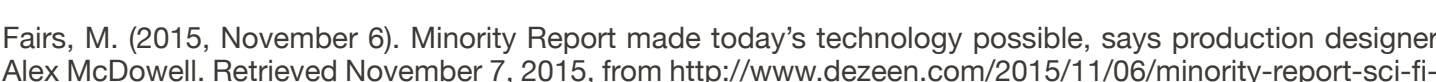
Alex McDowell. Retrieved November 7, 2015, from http:///www.dezeen.com/2015/11/06/minority-report-sci-fi-
movie-steven-spielberg-future-of-technology-predictions-possible-production-designer-alex-mcdowel//

Frederick, C. (1913). The new housekeeping efficiency studies in home management. Garden City, New York:

Hefner, H. M. (1969, March). The Playboy Interview: Marshall McLuhan. Playboy Magazine, 16(3).

Kaufmann, J. (2010). The meaning of cooking (English ed.). Cambridge, UK: Polity Press.

Kawulich, B. (2005). Participant Observation as a Data Collection Method. Forum: Qualitative Social Research,
6(2). Retrieved January 19, 2016, from http://www.qualitative-research.net/index.php/fqs/article/view/466/996 N. (2011). Counter space: Design and the modern kitchen. New York: Museum Kinchin, J., Con
of Modern Art.

Leach, H. (2010). From Kai to Kiwi kitchen: New Zealand's culinary traditions and cookbooks. Dunedin, New Zealand: Otago University Press.

Leach, H. (2014). Kitchens: The New Zealand kitchen in the 20th century. Dunedin, New Zealand: Otago

Lovell, S. (2011). Dieter Rams: As little design as possible. London: Phaidon.

Obal, Damjan, \& Stojmenova, Emilija. (2013). Experience to Understand. Springer Science \& Business Media,

Press, M., \& Cooper, R. (2003). The Design Experience: The Role of Design and Designers in the Twenty-Firs

Rouse, M. (2010, December). Pervasive Computing (Ubiquitous Computing). Retrieved February 14, 2016, from http://internetofthingsagenda.techtarget.com/definition/pervasive-computing-ubiquitous-computing

Rouse, M. (2015, July). Gesture Recognition. Retrieved February 22, 2016, from http://whatis.techtarget.com/ definition/gesture-recognition
Schöning, J., Rogers, Y., \& Krüger, A. (2012). Digitally Enhanced Food. IEEE Pervasive Computing, 11(3), 4-6.
Retrieved February 14, 2016. Sheppard, S. (2010, March). Trend Forecasting: Predicting the Outlook for Future Creative Thinking. Designe 源

Siegler, M. (2011, February 21). I Will Check My Phone At Dinner And You Will Deal With It. Tech Crunch. Retrieved February 10, 2016, from http://techcrunch.com/2011/02/21/phones-at-dinner/

Spence, Charles, \& Piqueras-Fiszman, Betina. (2013). Technology at the dining table. Oxford University, United Kingdom.

The Facts of Life: Generational Views About How We Live. (2015, November 17). Retrieved January 20, 2016 from http://www.nielsen.com/nz/en/insights/news/2015/the-facts-of-life-generational-views-about-how-we-

Turkle, S. (2015). Reclaiming conversation: The power of talk in a digital age. New York, New York: Penguin Press.

Winston, A. (2015, October 21). Back to the Future "brought the future closer" say designers. Retrieved cars-overboard-wearable-amb: $/$ nut-technology/. Vaananen-Vainio-Mattila, K., \& Koskela, T. (2004). Evolution towards smart home environments: Empirica

Wood, T. (2013, February 19). Experience Design: A Definition. Retrieved December 10, 2015.

FIGURES

All figures not cited here have been produced by the author.

Figure 2. 1863 Meeting of Settlers and Maoris at Hawke's Bay, New Zealand [Photograph]. (n.d.). The lllustrated 31) Figure 3. McMillan, A. (n.d.). Tree fern frond at Akatarawa. Retrieved February 27, 2016, from https:// (n) Retrieved February 26 Figure 4. Island Kitchen [Photograph found in Fisher\&Paykel Kitchen Tools]. (n.d.). Retrieved February 26,
2016 , from http//thekitchentools fisherpaykel com/case-study/sisand-kitchen/

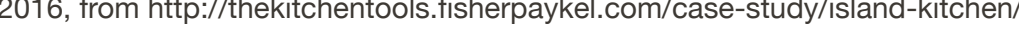

Figure 5. Smart Lock by August. (2013, November 19). Retrieved February 20, 2016, from http://techcrunch. com/2013/11/19/august-8m-maveron/

Figure 6. Ikea Future Kitchen 2025 Digital Dining. (2015, April 24). Retrieved February 20, 2016, from http:// Figure 7. Whirlpool Interactive Cooktop. (2014, January 8). Retrieved February 20, 2016, from http://www.
clairemontcommunications.com/pin-it-cook-it-eat-it/

Figure 9. Apple Watch - Guided Tour: Welcome [Video file]. (2015, April 3). Retrieved March 1, 2016, from https://www.youtube.com/watch?v=LHdVkPrdRYg

Figure 10. Minority Report gesture recognition. (2015, November 6). Retrieved February 24, 2016, from predictions-possible-production-designer-alex-mcdowell 
Figure 11. Back to the Future Part II Cinematic Release Poster. (n.d.). Retrieved February 15, 2016, from http:// Figure 12. Casa MJE by PKMN Architectures. (2015, October 4). Retrieved February 10, 2016, from http://
www.archdaily.Com/774668/mje-house-little-big-houses-number-2-pkmn-architectures

Figure 13. Fisher\&Paykel Gas Cooktop [Photograph]. (n.d.). In Fisher\&Paykel Image Bank. Retrieved February
10, 2016, from https://imagebank.fisherpaykel.com/. Figure 14. Fisher\&Paykel Cooldrawer [Photograph]. (n.d.). In Fisher\&Paykel Image Bank. Retrieved February
10, 2016, from https://imagebank. fisherpaykel.com/. Figure 15. Fisher\&Paykel Dishdrawer [Photograph]. (n.d.). In Fisher\&Paykel Image Bank. Retrieved February

Figure 16. PK11 Chair \& PK55 Table by Poul Kjærholm. (2014, July 26). Retrieved February 10, 2016, from http://designapplasse.com/news/people/floductons-from-fritz-hansen/42671/

Figure 17. Plank by Max Lamb. (2015, November 3). Retrieved February 10, 2016, from Casa MJJ by PKMN Architectures. (2015, October 4). Retrieved February 10, 2016, from http://www.archdaily.com/774668/mjehouse-little-big-houses-number-2-pkmn-architectures

Figure 18. Serif by Ronan and Erwan Bouroullec for Samsung. (2015, October 16). Retrieved February 10

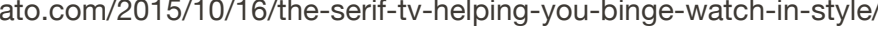

Figure 19. Multimedia Module by SieMatic. (n.d.). Retrieved February 10, 2016, from http://www.siematic.com/ Figure 20. Project Soli by Google. (n.d.). Retrieved February 10, 2016, from http://www.chicagotribune.com/

Figure 21. Mirror by Max Braun. (2016, February 2). Retrieved February 3, 2016, from http://www.technobuffalo. com/2016/02/02/google-engineer-built-the-most-beautiful-smart-mirror-ever/

Figure 22. Joe's Cheese Rolls. (2015, February 17). Retrieved February 10, 2016, from http://ourkitchen. isherpaykel.com/recipe/joes-cheese-rolls/ 
